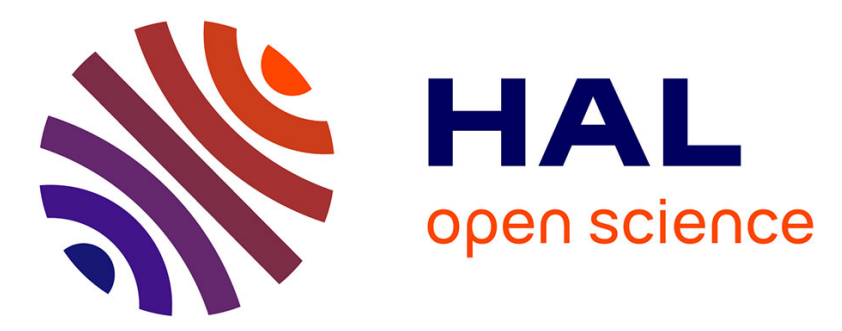

\title{
An explicit finite volume scheme on staggered grids for the Euler equations: Unstructured meshes, stability analysis, and energy conservation
}

Thierry Goudon, Julie Llobell, Sebastian Minjeaud

\section{- To cite this version:}

Thierry Goudon, Julie Llobell, Sebastian Minjeaud. An explicit finite volume scheme on staggered grids for the Euler equations: Unstructured meshes, stability analysis, and energy conservation. International Journal for Numerical Methods in Fluids, 2022, 94 (1), pp.76-119. 10.1002/fld.5048 . hal-03428367

\section{HAL Id: hal-03428367 https://hal.science/hal-03428367}

Submitted on 15 Nov 2021

HAL is a multi-disciplinary open access archive for the deposit and dissemination of scientific research documents, whether they are published or not. The documents may come from teaching and research institutions in France or abroad, or from public or private research centers.
L'archive ouverte pluridisciplinaire HAL, est destinée au dépôt et à la diffusion de documents scientifiques de niveau recherche, publiés ou non, émanant des établissements d'enseignement et de recherche français ou étrangers, des laboratoires publics ou privés. 


\title{
An explicit finite volume scheme on staggered grids for the Euler equations: unstructured meshes, stability analysis and energy conservation
}

\author{
Thierry Goudon*, Julie Llobell ${ }^{\dagger}$ Sebastian Minjeaud ${ }^{\ddagger}$ \\ Université Côte d'Azur, Inria, CNRS, LJAD \\ Parc Valrose, F-06108 Nice, France
}

\begin{abstract}
We set up a numerical strategy for the simulation of the Euler equations, in the framework of finite volume staggered discretizations where numerical densities, energies and velocities are stored on different locations. The main difficulty relies on the treatment of the total energy, which mixes quantities stored on different grids. The proposed method is strongly inspired, on the one hand, from the kinetic framework for the definition of the numerical fluxes, and, on the other hand, from the Discrete Duality Finite Volume (DDFV) framework, which has been designed for the simulation of elliptic equations on complex meshes. The time discretization is explicit and we exhibit stability conditions that guaranty the positivity of the discrete densities and internal energies. Moreover, while the scheme works on the internal energy equation, we can define a discrete total energy which satisfies a local conservation equation. We provide a set of numerical simulations to illustrate the behaviour of the scheme.
\end{abstract}

Keywords: Euler equations. Staggered grids. Discrete Duality Finite Volume.

\section{Contents}

1 Introduction $\quad 2$

2 Notation: meshes, unknowns 4

3 Definition of the scheme $\quad 7$

3.1 Mass conservation on the diamond cells . . . . . . . . . . . . . . . 9

3.2 Transfer lemma and mass conservation on primal and dual cells . . . . . . . . . 10

3.3 Momentum equation on the primal and dual cells . . . . . . . . . . . . . . . . 14

3.4 Internal energy balance on the diamond mesh . . . . . . . . . . . . . . . 15

3.5 Stability analysis . . . . . . . . . . . . . . . . . . . . . 19

4 Conservation of total energy 30

*thierry.goudon@inria.fr

$\dagger$ julie.llobell@unice.fr

${ }^{\ddagger}$ sebastian.minjeaud@unice.fr 
5.1 Consistency analysis with a 2D manufactured solution . . . . . . . . . . . . . . 34

5.2 Simulations of 1D Riemann problems . . . . . . . . . . . . . . . . . . . . . . . . . . . . 37

5.3 Simulations of $2 \mathrm{D}$ singular solutions . . . . . . . . . . . . . . . . . . 38

\section{Introduction}

This work is concerned with the simulation of the Euler system of gas dynamics

$$
\left\{\begin{array}{l}
\partial_{t} \rho+\nabla \cdot(\rho \mathbf{u})=0 \\
\partial_{t}(\rho \mathbf{u})+\nabla \cdot(\rho \mathbf{u} \otimes \mathbf{u})+\nabla p=0, \\
\partial_{t}(\rho E)+\nabla \cdot(\rho E \mathbf{u})+\nabla \cdot(p \mathbf{u})=0 .
\end{array}\right.
$$

The unknowns depend on the time and space variables $(t, x) \in[0, \infty) \times \Omega$ with $\Omega \subset \mathbb{R}^{2}$, a regular and bounded domain. In (1), $\rho, \mathbf{u}, E$ and $p$ stand for the mass density, the velocity field, the total energy and the pressure respectively. The pressure is related to the independent unknowns $(\rho, \mathbf{u}, E)$ through an equation of state that depends of the adiabatic exponent $\gamma>1$; in what follows we set

$$
E=\frac{\|\mathbf{u}\|^{2}}{2}+e \text { and } p=(\gamma-1) \rho e
$$

where $e$ is the internal energy.

The originality of our approach is to consider staggered grids, which means that the numerical unknowns are stored at different locations of the mesh. Consequently, in contrast to the usual approaches for the Euler equations, (1) is not treated as a system for the conserved quantities $(\rho, \rho \mathbf{u}, \rho E)$, but instead each equation is considered "independently" on its own grid. Therefore, the scheme is not based on resolution of local Riemann problems, but it uses only a relevant (and simple) notion of numerical fluxes, and the upwinding principles. This viewpoint is particularly motivated by the will to address simulations of complex models for mixtures which include an additional constraint on the velocity field, or the simulation of flows in low Mach regimes. It is indeed known that standard colocalized methods might lead to severe stability constraints, or simply fail in capturing the correct solutions in these regimes, see $[15,16,27,28]$. These issues (which would require some appropriate implicit time dicretization) are beyond the scope of the present paper: here, we focus on the preliminary step which consists in designing an efficient scheme for the Euler system on staggered grids.

This contribution takes place in a series of works, which started in [6] where the method is introduced for the barotropic Euler equation in one-dimension. In particular, the proposed scheme introduced numerical fluxes strongly inspired from the framework of kinetic schemes. The scheme is shown to be stable, under suitable CFL-conditions, it preserves the entropystructure and the consistency analysis à la Lax-Wendroff can be performed too [5]. This approach is further developped to handle complex systems for mixture flows [7]. The method has been adapted to deal with multi-dimensional equations, when working on Cartesian grids in [25]. It also includes MUSCL strategies that make the scheme second order accurate (for smooth solutions), for both the barotropic and the full Euler system. When considering the full Euler equations, we face the difficulty that the total energy mixes up quantities, typically densities/energies vs. velocities, naturally defined on different grids. It leads to work with the internal energy equation, at the price of taking into account appropriately the kinetic energy balance, as in [30,33]. Restricting to the barotropic case, the scheme has been adapted, still on Cartesian grids, to set up an asymptotic preserving method in the low Mach regimes [24]. In such a situation, the asymptotic regime leads to an incompressible limit. Accordingly the limit 
scheme correctly handles the incompressibility constraint: owing to the staggered strategy, the scheme has enforced stability/consistency properties, reminiscient of the MAC approach, in the spirit of the pioneering work of F. H. Harlow and J. E. Welch [29]. However, these versions of the scheme use strongly the Cartesian geometry of the grid. Here, we address the question of the design of the method on unstructured meshes, for the full Euler system. The main difficulty consists in finding consistent transfer procedures between the different grids, and a relevant approach for the energy equation, which cannot be treated by mere averages, due to the complex geometry of the mesh.

To this end, the adaptation we are going to discuss on unstructured grids is strongly inspired by the Discrete Duality Finite Volume (DDFV) framework. The DDFV framework has been introduced in the 2000s in $[18,35]$ to solve the Laplace equation on general 2D meshes, including non-conformal meshes, and, more generally, to numerically deal with elliptic operators $\boldsymbol{\nabla}$. $(A \boldsymbol{\nabla u}), x \mapsto A(x)$ being a matrix valued function. In the Finite Volume approach, one has to define numerical fluxes $A \boldsymbol{\nabla u} \cdot \mathbf{n}$ on the interfaces of the control volumes, and finding a relevant formula that uses only unknowns stored at the center of the control volumes is not possible without severe restrictions on the mesh geometry ${ }^{1}$. The DDFV approach has been extended to the Stokes equations in $[9,13,14,38]$ and to the Navier-Stokes equations in [21, $22,39,44]$. The main idea of the DDFV method is to introduce additional unknowns so that full gradients can be reconstructed, and to mimic at the discrete level the duality formula involving differential operators we are used to for continuous quantities. The Euler systems does not involve any elliptic operator, and it only involves first order derivatives. Nevertheless, the staggered scheme we propose gets its inspiration from the DDFV approach designed in [21] to solve the non homogeneous Navier-Stokes equations. We shall use ideas from [21] that consist in duplicating variables, together with a suitable treatment of the convection terms in order to restore the consistency for the equations on the primal and the dual meshes. We do not address this issue here, but we expect this approach to be well-adapted to handle low Mach regimes on unstructured meshes. Moreover, the duplication of variables can also open perspectives to reconstruct gradients and to design a second-order version of the scheme, in the spirit of [8] where a MUSCL scheme for the Euler equation is constructed based on DDFV principles.

The paper is organized as follows. In Section 2 we set up a few useful notations and make the definition of the different mesh-related quantities we are going to use precise. Section 3 details the construction of the scheme. Two ingredients are crucial. First, the definition of the mass fluxes, inspired from the kinetic framework, is very specific and induces several properties which play a central role in the stability-consistency analysis. Second, suitable footbridges should be introduced in order to transfer information from a grid to another, an issue pointed out in [4]. We pay attention to make such a transfer consistently, which relies on a general statement, as discussed in [23]. We shall equally discuss the stability analysis: under a suitable constraint on the time step, the scheme preserves the positivity of the mass density and of the internal energy. The constraint has the usual flavor of CFL conditions: the larger the material velocity and the sound speed and the finer the mesh, the smaller the time step; the constraint also involves some regularity coefficient associated with the mesh geometry. In Section 4, we turn to the balance of total energy. Precisely, we are able to provide a relevant definition of the discrete total energy and we justify that its time evolution satisfies a local conservation law. This desired statement can be seen as a consistency property of the scheme with the system of conservation laws, not obvious at first sight since the scheme works on a different version, non-conservative, of the equations. According to the recent works $[2,19,20,31]$, such a property could be the preliminary step to justify a consistency statement $\grave{a}$ la Lax-Wendroff [40]. Finally, Section 5 validates the scheme by a series of numerical experiments.

\footnotetext{
${ }^{1}$ if $x_{K}$ and $x_{L}$ stand for centers of adjacent control volumes, we only get an approximation of $\nabla v$ in the direction of $\left[x_{K}, x_{L}\right]$, while we need a full gradient to compute the flux $A \nabla \mathbf{u} \cdot \mathbf{n}$ when $A$ is a full matrix
} 


\section{Notation: meshes, unknowns}

From now on, we suppose that $\Omega$ is an open bounded polygonal domain of $\mathbb{R}^{2}$ and its boundary is denoted $\partial \Omega$.

Meshes. The construction uses three meshes: the primal mesh, the dual mesh and the diamond mesh; the main steps of the construction are illustrated by Fig. 1.

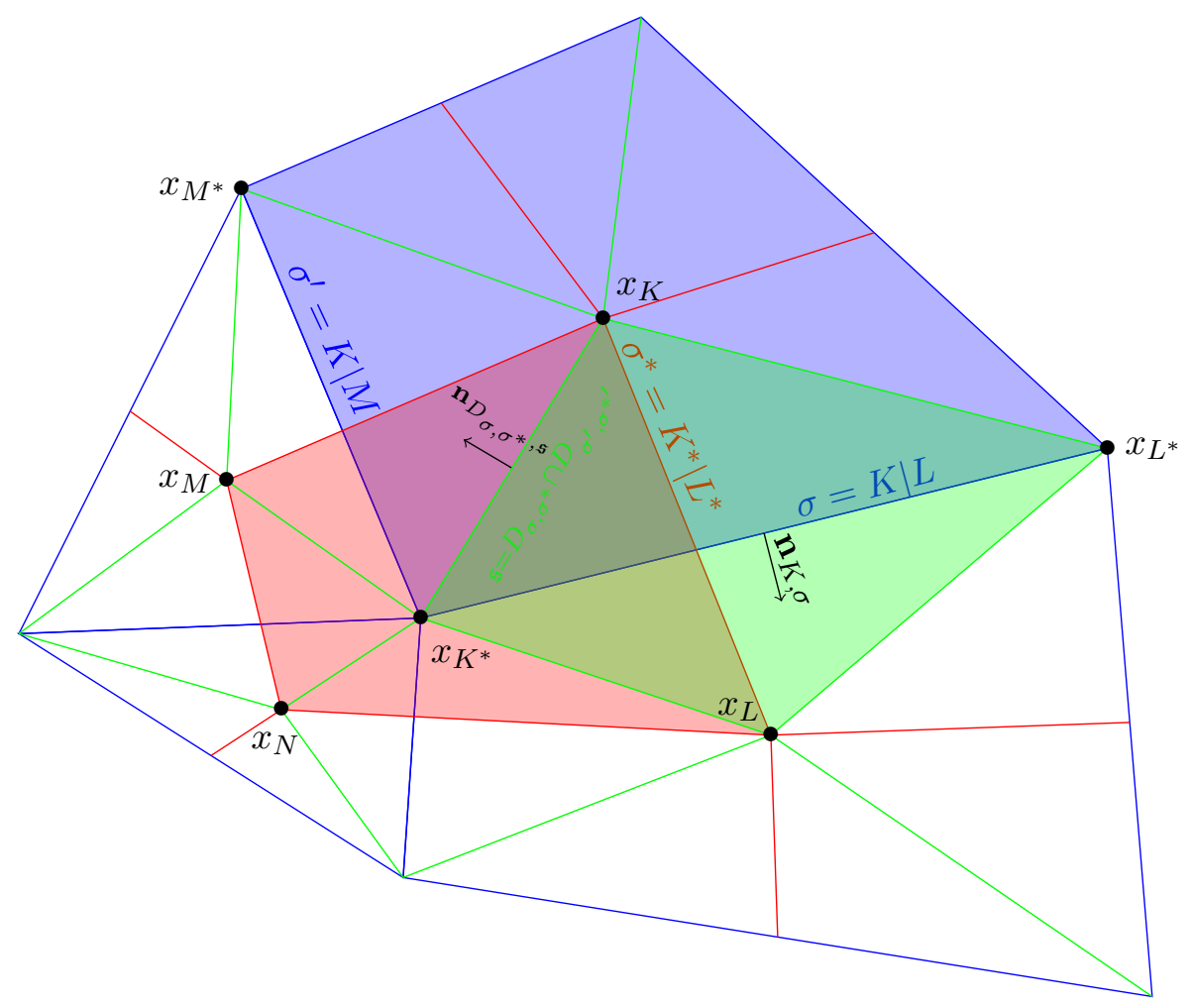

Figure 1: Meshes and associated notations.

- The primal mesh $\mathfrak{M}$ consists of disjoint, non-degenerate, convex polygons $K$ called "primal cells". We associate with each cell $K$ its barycenter $x_{K}$ (see the cells with blue edges in Fig. 1).

- The dual mesh $\mathfrak{M}^{*} \cup \partial \mathfrak{M}^{*}$ is made of cells built "around the vertices $x_{K^{*}}$ " of the primal mesh. See the cells with red edges in Fig. 1. There are several options to construct the dual mesh. To this end, it is convenient to distinguish the interior dual mesh $\mathfrak{M}^{*}$ associated to vertices $x_{K^{*}}$ which do not belong to $\partial \Omega$, and the boundary of the dual mesh $\partial \mathfrak{M}^{*}$ associated to $x_{K^{*}} \in \partial \Omega$. In what follows, we focus on the direct approach: the interior dual mesh $\mathfrak{M}^{*}$ consists of cells $K^{*}$ by joining the centers $x_{K}$ of all cells $K$ having $x_{K^{*}}$ as a vertex (see the red cell in Fig. 1). When $x_{K^{*}} \in \partial \Omega$, a dual cell $K^{*}$ is made by joining the centers $x_{K}$ of the cells $K$ that share the vertex $x_{K^{*}}$ and the centers of the two boundary edges containing $x_{K^{*}}$.

- The diamond mesh $\mathfrak{D}$ is made of quadrilateral cells $D_{\sigma, \sigma^{*}}$ obtained by joining the endpoints of the edges $\sigma=\left[x_{K^{*}}, x_{L^{*}}\right]$ of the primal mesh to the centers $x_{K}$ and $x_{L}$ of the primal mesh cells that share this edge, and we denote $\sigma^{*}=\left[x_{K}, x_{L}\right]$. See the cells with green edges in 
Fig. 1. We distinguish the diamonds of the boundary $\mathfrak{D}_{\text {ext }}=\left\{D_{\sigma, \sigma^{*}} \in \mathfrak{D}\right.$ such that $\sigma \in$ $\partial \Omega\}$ and $\mathfrak{D}_{\text {int }}=\mathfrak{D} \backslash \mathfrak{D}_{\text {ext }}$. In the specific case where $D_{\sigma, \sigma^{*}} \in \mathfrak{D}_{\text {ext }}$, the diamond cell $D_{\sigma, \sigma^{*}}$ degenerates to a triangle.

As explained above, we work with the direct dual mesh. From now on, we assume that the primal mesh is such that all associated diamonds are convex. In such a case the diagonals $\sigma=\left[x_{K^{*}}, x_{L^{*}}\right]$, and $\sigma^{*}=\left[x_{K}, x_{L}\right]$, which are equally edges of the primal - resp. dual - mesh, are included in the diamonds. In appendix A, we shall relax this assumption and consider an alternative construction of the dual mesh - the barycentrentric mesh - and we shall detail how the discussion can be adapted to this case.

Of course, the three meshes cover the computational domain:

$$
\Omega=\bigcup_{K \in \mathfrak{M}} K=\bigcup_{K^{*} \in \mathfrak{M}^{*} \cup \partial \mathfrak{M}^{*}} K^{*}=\bigcup_{D_{\sigma, \sigma^{*}} \in \mathfrak{D}} D_{\sigma, \sigma^{*}}
$$

The discretization is thus defined as a pair $(\mathfrak{T}, \mathfrak{D})$ where $\mathfrak{T}=\mathfrak{M} \cup \mathfrak{M}^{*} \cup \partial \mathfrak{M}^{*}$ combines the primal mesh $\mathfrak{M}$ and the dual mesh $\mathfrak{M}^{*} \cup \partial \mathfrak{M}^{*}$ and $\mathfrak{D}$ stands for the diamond mesh. Note that contrarily to standard DDFV notation, we do not introduce here the notation $\partial \mathfrak{M}$ for the set of edges of the primal mesh $\mathfrak{M}$ included in $\partial \Omega$, considered as degenerate cells.

Remark 2.1. The introduction of the three meshes $\mathfrak{M}, \mathfrak{M}^{*} \cup \partial \mathfrak{M}^{*}$ and $\mathfrak{D}$ is needed to explain the construction of the scheme and its analysis. However, at the end of the day, the obtained formula which defines the scheme uses only geometrical quantities (e.g. the measure of the diamond cells) and connectivities (e.g. the index number of control volume sharing a given edge) that can be easily computed from basic descriptors of the primal mesh. When implementing the scheme, these geometrical quantities are computed in a pre-processing step which is done once and for all for a given mesh.

Boundaries. For boundary conditions, we distinguish

- the zero-flux boundaries with nothing going out or going in at the interface between the domain and the outside,

- the outflow boundaries with no information coming from the outside of the domain,

- the Dirichlet boundaries where the variables are supposed to be known, equal to $\left(\rho_{\text {Dir }}, \mathbf{u}_{\text {Dir }}, e_{\text {Dir }}\right)$.

Notations. We refer the reader to Fig. 1 for the following notations.

- We denote $\sigma=K \mid L=\left[x_{K^{*}}, x_{L^{*}}\right]$ the edge separating two adjacent cells $K$ and $L$ of the primal mesh, and $\sigma^{*}=\left[x_{K}, x_{L}\right]$ is the segment that joins the centers of the cells $K$ and $L$. For the direct dual mesh, we have $\sigma^{*}=K^{*} \mid L^{*}$, the edge separating the adjacent cells $K^{*}$ and $L^{*}$ of the dual mesh.

- We denote $\mathfrak{s}=D_{\sigma, \sigma^{*}} \mid D_{\sigma^{\prime}, \sigma^{*^{\prime}}}$ the face separating two diamond cells $D_{\sigma, \sigma^{*}}$ and $D_{\sigma^{\prime}, \sigma^{*}}$.

- For $K \in \mathfrak{M}$, we denote $\mathfrak{D}_{K}=\left\{D_{\sigma, \sigma^{*}} \in \mathfrak{D}, \sigma \in \partial K\right\}$. For $K^{*} \in \mathfrak{M}^{*} \cup \partial \mathfrak{M}^{*}$, we similarly denote $\mathfrak{D}_{K^{*}}=\left\{D_{\sigma, \sigma^{*}} \in \mathfrak{D}, \sigma^{*} \in \partial K^{*}\right\}$.

- The area of a cell $X$ of $\mathfrak{M}, \mathfrak{M}^{*} \cup \partial \mathfrak{M}^{*}$ or $\mathfrak{D}$ is denoted $|X|$ and the length of an edge $\mathfrak{x}$ of type $\sigma, \sigma^{*}$ or $\mathfrak{s}$ is denoted $|\mathfrak{x}|$.

- We denote $h_{D_{\sigma, \sigma^{*}}}$ the diameter of the diamond cell $D_{\sigma, \sigma^{*}}$.

- For a cell $X$ of $\mathfrak{M}, \mathfrak{M}^{*} \cup \partial \mathfrak{M}^{*}$ or $\mathfrak{D}$ and for $\mathfrak{x} \in \partial X$, we define a unit vector $\mathbf{n}_{X, \mathfrak{x}}$ normal to the face $\mathfrak{x}$ of the cell $X$ and pointing outwards: $\mathbf{n}_{K, \sigma}$ (with $\sigma \in \partial K$ for $K \in \mathfrak{M}$ ), $\mathbf{n}_{K^{*}, \sigma^{*}}$ (with $\sigma^{*} \in \partial K^{*}$ for $K^{*} \in \mathfrak{M}^{*}$ ), and $\mathbf{n}_{D_{\sigma, \sigma^{*}, \mathfrak{s}}}\left(\right.$ with $\mathfrak{s} \in \partial D_{\sigma, \sigma^{*}}$ for $D_{\sigma, \sigma^{*}} \in \mathfrak{D}$ ). Note that, for $\sigma=K \mid L$, for $\sigma^{*}=K^{*} \mid L^{*}$ and for $\mathfrak{s}=D_{\sigma, \sigma^{*}} \mid D_{\sigma^{\prime}, \sigma^{*}}$, we have

$$
\mathbf{n}_{K, \sigma}=-\mathbf{n}_{L, \sigma}, \quad \mathbf{n}_{K^{*}, \sigma^{*}}=-\mathbf{n}_{L^{*}, \sigma^{*}}, \quad \mathbf{n}_{D_{\sigma, \sigma^{*}, \mathfrak{s}}}=-\mathbf{n}_{D_{\sigma^{\prime}, \sigma^{*}, \mathfrak{s}}}
$$


- Given $s \in \mathbb{R}$, we denote $[s]^{+}=\max (s, 0)=\frac{|s|+s}{2}$, and $[s]^{-}=\max (-s, 0)=\frac{|s|-s}{2}$, its positive and negative parts, respectively. Note they are both non negative $[s]^{ \pm} \geq 0$ and that $s=[s]^{+}-[s]^{-},|s|=[s]^{+}+[s]^{-}$.

- In order to analyze the preservation of the non negativity of the density and the internal energy, we need to introduce a positive number reg $(\mathfrak{T})$ that measures the regularity of the mesh $\mathfrak{M}$ :

$$
\begin{aligned}
\operatorname{reg}(\mathfrak{T})=\sup & \left(\left\{\frac{1}{\sin \left(\alpha_{D_{\sigma, \sigma^{*}}}\right)}, D_{\sigma, \sigma^{*}} \in \mathfrak{D}\right\} \cup\left\{\frac{h_{D_{\sigma, \sigma^{*}}}}{\sqrt{\left|D_{\sigma, \sigma^{*}}\right|}}, D_{\sigma, \sigma^{*}} \in \mathfrak{D}\right\}\right. \\
& \left.\cup\left\{\frac{\left|D_{\sigma, \sigma^{*}}\right|}{\left|D_{\sigma, \sigma^{*}} \cap X\right|}, X \in \mathfrak{T}, D_{\sigma, \sigma^{*}} \in \mathfrak{D}_{X}\right\} \cup\left\{\frac{|X|}{\left|D_{\sigma, \sigma^{*}}\right|}, X \in \mathfrak{T}, D_{\sigma, \sigma^{*}} \in \mathfrak{D}_{X}\right\}\right),
\end{aligned}
$$

where $\alpha_{D_{\sigma, \sigma^{*}}}$ is the angle in $(0, \pi / 2]$ between the two diagonals of the diamond cell $D_{\sigma, \sigma^{*}}$. Given two internal cells $K$ and $L$ with a common interface $\sigma=K \mid L$, the diamond $D_{\sigma, \sigma^{*}}$ can be cast as the reunion $\left(D_{\sigma, \sigma^{*}} \cap K\right) \cup\left(D_{\sigma, \sigma^{*}} \cap L\right)$ and $\frac{\left|D_{\sigma, \sigma^{*}}\right|}{\left|D_{\sigma, \sigma^{*}} \cap K\right|}=1+\frac{\left|D_{\sigma, \sigma^{*}} \cap L\right|}{\left|D_{\sigma, \sigma^{*}} \cap K\right|}$, so that $\sup \left(\frac{\left|D_{\sigma, \sigma^{*}}\right|}{\left|D_{\sigma, \sigma^{*}} \cap X\right|}, X \in\{K, L\}\right) \geq 2$ and accordingly $\operatorname{reg}(\mathfrak{T}) \geq 2$. The quantity reg $(\mathfrak{T})$ measures the regularity of the mesh, the higher $\operatorname{reg}(\mathfrak{T})$, the flatter the cells. It is known that we can find a constant $C$ which depends only on reg $(\mathfrak{T})$ such that, for any diamond cell $D_{\sigma, \sigma^{*}}$ and for any edge $\mathfrak{s}$ of $D_{\sigma, \sigma^{*}}$,

$$
\frac{h_{D_{\sigma, \sigma^{*}}}}{C} \leqslant|\sigma| \leqslant C h_{D_{\sigma, \sigma^{*}}}, \quad \frac{h_{D_{\sigma, \sigma^{*}}}}{C} \leqslant\left|\sigma^{*}\right| \leqslant C h_{D_{\sigma, \sigma^{*}}}, \quad \frac{h_{D_{\sigma, \sigma^{*}}}}{C} \leqslant|\mathfrak{s}| \leqslant C h_{D_{\sigma, \sigma^{*}}},
$$

and

$$
\frac{h_{D_{\sigma, \sigma^{*}}}^{2}}{C} \leqslant\left|D_{\sigma, \sigma^{*}}\right| \leqslant C h_{D_{\sigma, \sigma^{*}}}^{2} .
$$

\section{Unknowns.}

- Density, internal energy and pressure are piecewise constants over the diamond cells; alternatively, we can think of quantities stored on the edges of the initial mesh: $\rho_{\sigma, \sigma^{*}}$ and $e_{\sigma, \sigma^{*}}$ are constant on the diamond cell $D_{\sigma, \sigma^{*}} \in \mathfrak{D}$ and we set $p_{\sigma, \sigma^{*}}=(\gamma-1) \rho_{\sigma, \sigma^{*}} e_{\sigma, \sigma^{*}}$.

- The numerical velocity fields are piecewise constants over the primal and dual cells, stored at both the centers and the vertices of the cell of the primal mesh: $\mathbf{u}_{K}$ is constant on the primal cell $K \in \mathfrak{M}$ and $\mathbf{u}_{K^{*}}$ is constant on the dual cell $K^{*} \in \mathfrak{M}^{*} \cup \partial \mathfrak{M}^{*}$.

Observe that, in contrast to the Cartesian framework studied in [25], we store all the components of the velocity on the centers and vertices of the primal meshes. The Cartesian case is less demanding in terms of storage since the geometry allows us to store only the horizontal or the vertical component at a given location, in the same fashion as the MAC discretization for incompressible flows.

It is finally convenient to introduce further quantities, related to the internal energy and the velocity, that are stored at the edges of the diamond mesh and at the boundary edges of the primal mesh.

Definition 2.2. For $\mathfrak{s}=D_{\sigma, \sigma^{*}} \mid D_{\sigma^{\prime}, \sigma^{* \prime}}$, we denote

$$
e_{\mathfrak{s}}:=\frac{e_{\sigma, \sigma^{*}}+e_{\sigma^{\prime}, \sigma^{*^{\prime}}}}{2} .
$$

For $\mathfrak{s}=\left[x_{K}, x_{K^{*}}\right]$ an edge of $D_{\sigma, \sigma^{*}}$, we denote

$$
u_{D_{\sigma, \sigma^{*}, \mathfrak{s}}}:=\frac{\mathbf{u}_{K}+\mathbf{u}_{K^{*}}}{2} \cdot \mathbf{n}_{D_{\sigma, \sigma^{*}, \mathfrak{s}}} .
$$


For $\sigma=\left[x_{K^{*}}, x_{L^{*}}\right]$ an edge of $K$ such that $\sigma \subset \partial \Omega$, we denote

$$
\begin{aligned}
& e_{\sigma}= \begin{cases}e_{\sigma, \sigma^{*}} & \text { if } \sigma \text { is a zero-flux or an outflow boundary, } \\
e_{\text {Dir }} & \text { if } \sigma \text { is a Dirichlet boundary, }\end{cases} \\
& \mathbf{u}_{\sigma}= \begin{cases}0 & \text { if } \sigma \text { is a zero-flux boundary, } \\
\frac{\mathbf{u}_{K^{*}}+\mathbf{u}_{L^{*}}}{2} & \text { if } \sigma \text { is an outflow boundary, } \\
\mathbf{u}_{\text {Dir }} & \text { if } \sigma \text { is a Dirichlet boundary, }\end{cases}
\end{aligned}
$$

and

$$
u_{\sigma}:=\mathbf{u}_{\sigma} \cdot \mathbf{n}_{K, \sigma}
$$

Similarly, we denote $u_{\text {Dir }}:=\mathbf{u}_{\text {Dir }} \cdot \mathbf{n}_{K, \sigma}$ when $\sigma$ is a Dirichlet boundary.

Note that

$$
u_{D_{\sigma, \sigma^{*}, \mathfrak{s}}}=-u_{D_{\sigma^{\prime}, \sigma^{*}}, \mathfrak{s}} \quad \text { if } \quad \mathfrak{s}=D_{\sigma, \sigma^{*}} \mid D_{\sigma^{\prime}, \sigma^{*^{\prime}}}
$$

Remark 2.3. This discretisation technique differs from the staggered approach developed in [30, $33]$ : dealing with grids made of quadrilaterals in dimension 2, in [30, 33] densities and pressures are stored at the center of the control volumes and, using ideas reminiscent to RannacherTurek or Crouzeix-Ravart finite element methods, velocities are stored at the center of the faces of the mesh. The corresponding scheme can be shown to conserve globally the total energy, and stability/consistency properties are further discussed in $[30,33]$. While the method differs by many aspects (discretization, definition of the numerical mass and momentum fluxes), the analysis of our scheme is based on manipulations close to the proofs of [30, 33].

In what follows, we shall repeatedly use the following elementary claim.

Lemma 2.4. Consider a triangle $A B C$. For a given vertex $V$, we denote $|v|$ the length of the edge $v$ that does not contain $V$ and $\mathbf{n}_{V}$ stands for the outward unit vector, see Fig. 2. The following equality holds: $|a| \mathbf{n}_{A}+|b| \mathbf{n}_{B}+|c| \mathbf{n}_{C}=0$.

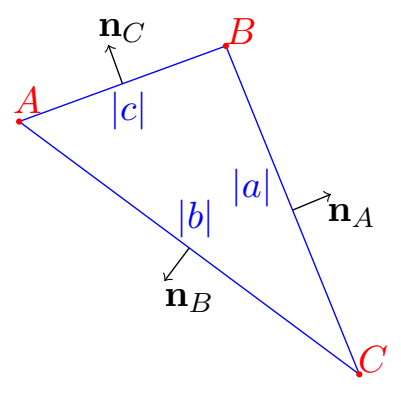

Figure 2: Triangle $A B C$

\section{Definition of the scheme}

For further purposes, we remind the reader that the sound speed of (1) is

$$
c(e)=\sqrt{\gamma(\gamma-1) e},
$$

which only depends on the internal energy $e$. This quantity naturally enters in the definition of the numerical fluxes since it is related to the speed of propagation of the information as driven 
by (1). Indeed, let us write (1) in the non-conservative form

$$
\left\{\begin{array}{l}
\partial_{t} \rho+\mathbf{u} \cdot \boldsymbol{\nabla} \rho+\rho \boldsymbol{\nabla} \cdot \mathbf{u}=0 \\
\partial_{t} \mathbf{u}+(\mathbf{u} \cdot \boldsymbol{\nabla}) \mathbf{u}+\rho^{-1} \boldsymbol{\nabla} p=0 \\
\partial_{t} e+\mathbf{u} \cdot \boldsymbol{\nabla} e+\rho^{-1} p \boldsymbol{\nabla} \cdot \mathbf{u}=0
\end{array}\right.
$$

Denoting $U=(\rho, \mathbf{u}, e)$, it can be cast in matrix from as $\partial_{t} U+A(U, \boldsymbol{\nabla}) U=0$ with

$$
A(U, \boldsymbol{\nabla})=\left(\begin{array}{ccc}
\mathbf{u} \cdot \boldsymbol{\nabla} & \rho \boldsymbol{\nabla} \cdot & 0 \\
\left.\rho^{-1} \frac{\partial p}{\partial \rho}\right|_{e} \boldsymbol{\nabla} & \mathbf{u} \cdot \boldsymbol{\nabla} & \left.\rho^{-1} \frac{\partial p}{\partial e}\right|_{\rho} \boldsymbol{\nabla} \\
0 & \rho^{-1} p \boldsymbol{\nabla} \cdot & u \cdot \boldsymbol{\nabla}
\end{array}\right) .
$$

Let $\xi \in \mathbb{R}^{2}$ with $\|\xi\|=1$. Then, denoting $u=\mathbf{u} \cdot \xi, \lambda_{-}(u, c)=u-c, u$ and $\lambda_{+}(u, c)=u+c$ are the eigenvalues of the matrix $A(U, \xi)$ and they will play a central role in the construction of the scheme.

It is well-known that the conservative and non-conservative formulations of the Euler system are equivalent only for smooth solutions. As far as the solutions are smooth, we can indeed substract the kinetic energy balance from the equation for the total energy, which leads to the evolution equation for the internal energy

$$
\partial_{t}(\rho e)+\nabla \cdot(\rho e \mathbf{u})+p \boldsymbol{\nabla} \cdot \mathbf{u}=0 .
$$

This equality does not hold for discontinuous solutions, in which case, additionally, the product of the pressure $p$ with $\boldsymbol{\nabla} \cdot \mathbf{u}$ can be misleading.

This is the reason why Eulerian numerical methods are often based on discretized versions of (1). However, working with Eulerian staggered discretizations raises the issue of the definition of the total energy: the kinetic energy $\frac{\left\|\mathbf{u}_{K}\right\|^{2}}{2}$, the density $\rho_{\sigma, \sigma^{*}}$, and internal energy $e_{\sigma, \sigma^{*}}$ are defined on different grids. In this perspective, it would be natural to make use of (2) to update the internal energy [30, 33].

Note that Lagrangian approaches are also inspired by the non-conservative form, with grid displacements based on the material velocity and a projection step to go back to a fixed Eulerian grid, see [17, Chap. 5] for an introduction. Inspired by the seminal work [52], there exists staggered versions of Lagrangian methods, including high order schemes: see $[1,12,41]$ and the references therein for recent developments.

Let us explain the basis of our approach. We have at hand discrete density $\rho_{\sigma, \sigma^{*}}$, internal energy $e_{\sigma, \sigma^{*}}$ and velocities $\mathbf{u}_{K}, \mathbf{u}_{K^{*}}$. We denote by $\bar{z}$ the update of the quantity $z$ over a time step. We start with the discrete mass conservation, which takes the form

$$
\frac{\bar{\rho}_{\sigma, \sigma^{*}}-\rho_{\sigma, \sigma^{*}}}{\delta t}+\frac{1}{\left|D_{\sigma, \sigma^{*}}\right|} \sum_{\mathfrak{s} \in \partial D_{\sigma, \sigma^{*}}}|\mathfrak{s}| \mathcal{F}_{D_{\sigma, \sigma^{*}, \mathfrak{s}}}=0
$$

where $\delta t$ stands for the time step and $\mathcal{F}_{D_{\sigma, \sigma^{*}, \mathfrak{s}}}$ are the discrete mass fluxes that will be defined in Section 3.1. From this, we can define a discrete density $\rho_{K}$ on the primal mesh, and we update the momentum on primal mesh with a formula which looks like

$$
\frac{\bar{\rho}_{K} \overline{\mathbf{u}}_{K}-\rho_{K} \mathbf{u}_{K}}{\delta t}+\frac{1}{|K|} \sum_{D_{\sigma, \sigma} \in \mathfrak{D}_{K}}|\sigma| \mathcal{G}_{K, \sigma}+\left(\boldsymbol{\nabla}_{\boldsymbol{d}} p\right)_{K}=0
$$

The momentum fluxes $\mathcal{G}_{K, \sigma}$ correspond to the discretization of $\boldsymbol{\nabla} \cdot(\rho \mathbf{u} \otimes \mathbf{u})$; they will be defined from suitable average of the mass fluxes $\mathcal{F}_{D_{\sigma, \sigma^{*}, \mathfrak{s}}}$, according to the conservation principles of the 
equations. The discrete pressure gradient $\left(\boldsymbol{\nabla}_{\boldsymbol{d}} p\right)_{K}$ stands for a discrete version of $\boldsymbol{\nabla} p$. We also construct a similar formula for updating $\rho_{K^{*}} \mathbf{u}_{K^{*}}$. Finally, we update the internal energy with the following discrete version of (2)

$$
\frac{\bar{\rho}_{\sigma, \sigma^{*}} \bar{e}_{\sigma, \sigma^{*}}-\rho_{\sigma, \sigma^{*}} e_{\sigma, \sigma^{*}}}{\delta t}+\frac{1}{\left|D_{\sigma, \sigma^{*}}\right|} \sum_{\mathfrak{s} \in \partial D_{\sigma, \sigma^{*}}}|\mathfrak{s}| \mathcal{E}_{D_{\sigma, \sigma^{*}, \mathfrak{s}}}+p_{\sigma, \sigma^{*}}\left(\boldsymbol{\nabla}_{\boldsymbol{d}} \cdot \overline{\mathbf{u}}\right)_{\sigma, \sigma^{*}}=\mathcal{R}_{\sigma, \sigma^{*}}
$$

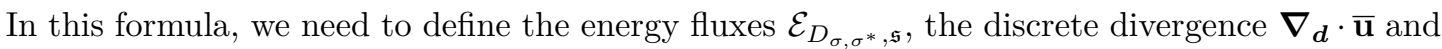
the correction term $\mathcal{R}_{\sigma, \sigma^{*}}$ which should keep track of possible discontinuities. The construction of the latter is inspired from [30,33]. Similar ideas are applied in the context of Lagrangian methods in [12]. We shall pay attention to the design of the former, in order to preserve the consistency with the local conservation of the total energy. It relies on suitable definitions of the fluxes and duality relation between the discrete operators $\boldsymbol{\nabla}_{\boldsymbol{d}} \cdot \mathbf{u}$ and $\boldsymbol{\nabla}_{\boldsymbol{d}} p$. Note that these formula also need to be adapted to handle boundaries of the computational domain.

\subsection{Mass conservation on the diamond cells}

We start by defining the mass fluxes on the interfaces of the diamond cell. The construction of the fluxes proposed in [6] involves the following function, parametrized by $\rho \geqslant 0, c \geqslant 0, u \in \mathbb{R}$,

$$
\xi \in \mathbb{R} \longmapsto \mathcal{M}_{[\rho, c, u]}(\xi)=\frac{\rho}{2 c} \mathbb{1}_{|\xi-u| \leqslant c},
$$

which has a compact support, precisely limited by the characteristic speeds of the Euler system. This function arises in the definition of kinetic schemes for solving the Euler system [37], and the support property plays a crucial role in the stability analysis of such schemes $[47,48]$. The numerical fluxes are defined by using this function, accounting for both a direction of propagation and the characteristic speeds.

Definition 3.1. Let

$$
\mathcal{F}^{+}(\rho, c, u)=\int_{\xi>0} \xi \mathcal{M}_{[\rho, c, u]}(\xi) d \xi= \begin{cases}0 & \text { if } u \leqslant-c \\ \frac{\rho}{4 c} \lambda_{+}(u, c)^{2} & \text { if }|u| \leqslant c \\ \rho u & \text { if } u \geqslant c\end{cases}
$$

and

$$
\mathcal{F}^{-}(\rho, c, u)=\int_{\xi<0} \xi \mathcal{M}_{[\rho, c, u]}(\xi) d \xi= \begin{cases}\rho u & \text { if } u \leqslant-c, \\ -\frac{\rho}{4 c} \lambda_{-}(u, c)^{2} & \text { if }|u| \leqslant c \\ 0 & \text { if } u \geqslant c .\end{cases}
$$

It is worth pointing out that, despite its "kinetic" flavor, the definition of the flux function has a very simple expression and does not need any numerical computation of integrals. The following properties are fundamental for analysing the scheme $[5,6,7,25]$.

Lemma 3.2. The functions $\mathcal{F}^{ \pm}$satisfy the following properties

- symmetry :

$$
\mathcal{F}^{-}(\rho, c, u)=-\mathcal{F}^{+}(\rho, c,-u)
$$

- consistency :

$$
\mathcal{F}^{+}(\rho, c, u)+\mathcal{F}^{-}(\rho, c, u)=\rho u,
$$

- for any $u \in \mathbb{R}, \rho \geqslant 0$ and $c \geqslant 0$, we have

$$
0 \leqslant \mathcal{F}^{+}(\rho, c, u) \leqslant \rho\left[\lambda_{+}(c, u)\right]^{+} \quad \text { and } \quad-\rho\left[\lambda_{-}(c, u)\right]^{-} \leqslant \mathcal{F}^{-}(\rho, c, u) \leqslant 0 .
$$


The scheme is next based on the upwinding principles applied to the expression

$$
\mathcal{F}^{ \pm}=\int_{\xi \lessgtr 0} \xi \mathcal{M} \mathrm{d} \xi
$$

Namely, given an interface, the mass flux associated to the positive (resp. negative) kinetic velocities $\xi$ uses the backward (frontward) density. This definition significantly differs from the scheme introduced in $[30,32,33]$ which is based instead on the material velocity $u$ only (and not on the characteristic speeds), in the spirit of AUSM schemes [43, 42]. It induces naturally some numerical diffusion which prevents the formation of oscillations when the material $u$ velocity becomes small, see [6, Appendix B].

We thus define the mass flux $\mathcal{F}_{D_{\sigma, \sigma^{*}, \mathfrak{s}}}$ from the diamond cell $D_{\sigma, \sigma^{*}}$ through the interface $\mathfrak{s}=D_{\sigma, \sigma^{*}} \mid D_{\sigma^{\prime}, \sigma^{*^{\prime}}}$ as follows

$$
\mathcal{F}_{D_{\sigma, \sigma^{*}, \mathfrak{s}}}=\mathcal{F}_{D_{\sigma, \sigma^{*}, \mathfrak{s}}}^{+}+\mathcal{F}_{D_{\sigma, \sigma^{*}, \mathfrak{s}}}^{-}
$$

with

$$
\mathcal{F}_{D_{\sigma, \sigma^{*}, \mathfrak{s}}}^{+}=\mathcal{F}^{+}\left(\rho_{\sigma, \sigma^{*}}, c\left(e_{\mathfrak{s}}\right), u_{D_{\sigma, \sigma^{*}, \mathfrak{s}}}\right) \quad \text { and } \quad \mathcal{F}_{D_{\sigma, \sigma^{*}, \mathfrak{s}}}^{-}=\mathcal{F}^{-}\left(\rho_{\sigma^{\prime}, \sigma^{*}}, c\left(e_{\mathfrak{s}}\right), u_{D_{\sigma, \sigma^{*}, \mathfrak{s}}}\right) .
$$

It uses the velocity $u_{D_{\sigma, \sigma^{*}, \mathfrak{s}}}$ and the sound speed $c\left(e_{\mathfrak{s}}\right)$ naturally given on the interface by Definition 2.2, and upwinds the density. The symmetry property (5) implies that

$$
\mathcal{F}_{D_{\sigma, \sigma^{*}, \mathfrak{s}}}=-\mathcal{F}_{D_{\sigma^{\prime}, \sigma^{*}}, \mathfrak{s}}
$$

and thus $\mathcal{F}_{D_{\sigma, \sigma^{*}, \mathfrak{s}}}$ is a conservative flux. Moreover we have the following two equalities:

$$
\mathcal{F}_{D_{\sigma, \sigma^{*}, \mathfrak{s}}}^{+}=-\mathcal{F}_{D_{\sigma^{\prime}, \sigma^{*}, \mathfrak{s}}^{-}} \quad \text { and } \quad \mathcal{F}_{D_{\sigma, \sigma^{*}, \mathfrak{s}}}^{-}=-\mathcal{F}_{D_{\sigma^{\prime}, \sigma^{*}}^{+}} \cdot
$$

The discrete mass equation on a cell $D_{\sigma, \sigma^{*}} \in \mathfrak{D}_{\text {int }}$ is given by

$$
\frac{\bar{\rho}_{\sigma, \sigma^{*}}-\rho_{\sigma, \sigma^{*}}}{\delta t}+\frac{1}{\left|D_{\sigma, \sigma^{*}}\right|} \sum_{\mathfrak{s} \in \partial D_{\sigma, \sigma^{*}}}|\mathfrak{s}| \mathcal{F}_{D_{\sigma, \sigma^{*}, \mathfrak{s}}}=0 .
$$

The definition needs to be slightly adapted at the boundary. For the diamond cell $D_{\sigma, \sigma^{*}} \in$ $\mathfrak{D}_{\text {ext }}$, we have to define the outgoing mass flux $\mathcal{F}_{\sigma}=\mathcal{F}_{\sigma}^{+}+\mathcal{F}_{\sigma}^{-}$through the boundary edge $\sigma$. Denoting $K$ the primal cell whose $\sigma$ is an edge, we adopt the following definition:

- $\mathcal{F}_{\sigma}^{+}=0$ and $\mathcal{F}_{\sigma}^{-}=0$ for zero-flux conditions,

- $\mathcal{F}_{\sigma}^{+}=\mathcal{F}^{+}\left(\rho_{\sigma, \sigma^{*}}, c\left(e_{\sigma}\right), u_{\sigma}\right)$ and $\mathcal{F}_{\sigma}^{-}=0$ for outflow conditions,

- $\mathcal{F}_{\sigma}^{+}=\mathcal{F}^{+}\left(\rho_{\sigma, \sigma^{*}}, c\left(e_{\sigma}\right), u_{\sigma}\right)$ and $\mathcal{F}_{\sigma}^{-}=\mathcal{F}^{-}\left(\rho_{\text {Dir }}, c\left(e_{\sigma}\right), u_{\sigma}\right)$ for Dirichlet conditions.

The boundary quantities $u_{\sigma}$ and $c\left(e_{\sigma}\right)$ are given in Definition 2.2.

The discrete mass equation on a cell $D_{\sigma, \sigma^{*}} \in \mathfrak{D}_{\text {ext }}$ is given by

$$
\frac{\bar{\rho}_{\sigma, \sigma^{*}}-\rho_{\sigma, \sigma^{*}}}{\delta t}+\frac{1}{\left|D_{\sigma, \sigma^{*}}\right|} \sum_{\mathfrak{s} \in \partial D_{\sigma, \sigma^{*}} \backslash \partial \Omega}|\mathfrak{s}| \mathcal{F}_{D_{\sigma, \sigma^{*}, \mathfrak{s}}}+\frac{|\sigma|}{\left|D_{\sigma, \sigma^{*}}\right|} \mathcal{F}_{\sigma}=0 .
$$

\subsection{Transfer lemma and mass conservation on primal and dual cells}

In order to transfer the information from a grid to another, we shall make use of the following transfer lemma, extracted from [23]. 
Lemma 3.3. Consider a cell $\mathscr{C}$ and for each edge $\mathfrak{s}$ of $\mathscr{C}$, with unit outward normal $\mathbf{n}_{\mathscr{C}, \mathfrak{s}}$, we consider a given flux-type quantity $\mathcal{X}_{\mathscr{C}, \mathfrak{s}}$. There exists a function $\omega_{\mathscr{C}}$, which lies in $H_{\mathrm{div}}$, such that

$$
\nabla \cdot \omega_{\mathscr{C}}=\frac{1}{|\mathscr{C}|} \sum_{\mathfrak{s} \in \partial \mathscr{C}}|\mathfrak{s}| \mathcal{X}_{\mathscr{C}, \mathfrak{s}}
$$

and

$$
\int_{\mathfrak{s}} \omega_{\mathscr{C}} \cdot \mathbf{n}_{\mathscr{C}, \mathfrak{s}}=|\mathfrak{s}| \mathcal{X}_{\mathscr{C}, \mathfrak{s}} .
$$

The question of designing fluxes in order to maintain consistency between different meshes has been addressed in [4]. Lemma 3.3 appeared in [4] where solutions were proposed in the framework of Crouzeix-Raviart and Rannacher-Turek discretizations. It also appeared in [21], in the specific case of quadrilateral and convex cells, as a key ingredient for designing convection fluxes for the incompressible Navier-Stokes equation with variable density; it has been generalized in [23], which, furthermore, provides practical procedures to compute the transfer formula. This statement will be used in different places. First, we need it in order to define the momentum fluxes to be used for updating the velocity. To this end, we apply Lemma 3.3 on the diamond cells $\mathscr{C}=D_{\sigma, \sigma^{*}} \in \mathfrak{D}$. This allows us to define numerical densities on the primal and dual meshes, together with numerical conservative mass fluxes, so that a discrete mass conservation holds on these meshes too. Second, we will work the other way around, with $\mathscr{C}=K$ or $K^{*}$ in Section 4 in order to justify the local conservation of the total energy.

Let us explain how this works on the diamond cells $\mathscr{C}=D_{\sigma, \sigma^{*}} \in \mathfrak{D}$. We remind the reader that the edges $\mathfrak{s}$ of the diamond cell $D_{\sigma, \sigma^{*}}$ are of the form $\mathfrak{s}_{X Z^{*}}=\left[x_{X}, x_{Z^{*}}\right]$ with $\left(X, Z^{*}\right) \in\left\{\left(K, K^{*}\right),\left(L, K^{*}\right),\left(L, L^{*}\right),\left(K, L^{*}\right)\right\}$, see Fig. 3, and at each edge is associated a mass flux $\mathcal{F}_{D_{\sigma, \sigma^{*}, \mathfrak{s}}}$. Thus, Lemma 3.3 gives the existence of a function $\omega_{D_{\sigma, \sigma^{*}}} \in H_{\mathrm{div}}$ such that

$$
\nabla \cdot \omega_{D_{\sigma, \sigma^{*}}}=\frac{1}{\left|D_{\sigma, \sigma^{*}}\right|} \sum_{\mathfrak{s} \in \partial D_{\sigma, \sigma^{*}}}|\mathfrak{s}| \mathcal{F}_{D_{\sigma, \sigma^{*}, \mathfrak{s}}},
$$

and

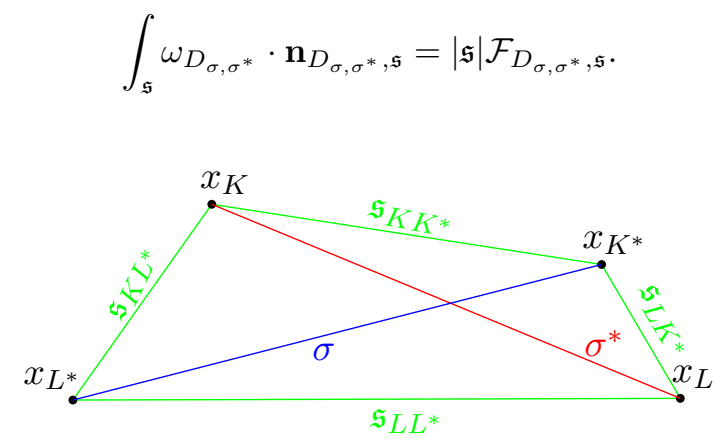

Figure 3: Diamond cell $D_{\sigma, \sigma^{*}}$.

Since the diagonal $\sigma$ is an edge of the primal mesh, say for the cell $K$, we get a mass flux by setting

$$
|\sigma| \mathcal{F}_{K, \sigma}=\int_{\sigma} \omega_{D_{\sigma, \sigma^{*}}} \cdot \mathbf{n}_{K, \sigma}
$$


This quantity has actually a simple expression by means of the original mass fluxes $\mathcal{F}_{D_{\sigma, \sigma^{*}, \mathfrak{F}}}$. Indeed, we have

$$
\begin{aligned}
|\sigma| \mathcal{F}_{K, \sigma} & =\int_{D_{\sigma, \sigma^{*}} \cap K} \nabla \cdot \omega_{D_{\sigma, \sigma^{*}}}-\int_{\mathfrak{s}_{K K^{*}}} \omega_{D_{\sigma, \sigma^{*}}} \cdot \mathbf{n}_{\mathfrak{s}_{K K^{*}}}-\int_{\mathfrak{s}_{K L^{*}}} \omega_{D_{\sigma, \sigma^{*}}} \cdot \mathbf{n}_{\mathfrak{s}_{K L^{*}}} \\
& =\frac{\left|D_{\sigma, \sigma^{*}} \cap K\right|}{\left|D_{\sigma, \sigma^{*}}\right|} \sum_{\mathfrak{s} \in \partial D_{\sigma, \sigma^{*}}}|\mathfrak{s}| \mathcal{F}_{D_{\sigma, \sigma^{*}, \mathfrak{s}}}-\left|\mathfrak{s}_{K K^{*}}\right| \mathcal{F}_{\sigma, \mathfrak{s}_{K K^{*}}}-\left|\mathfrak{s}_{K L^{*}}\right| \mathcal{F}_{\sigma, \mathfrak{s}_{K L^{*}}} .
\end{aligned}
$$

Using the obvious relation $|D|=|D \cap K|+|D \cap L|$, for the interface $\sigma=K \mid L$ we finally arrive at

$$
\mathcal{F}_{K, \sigma}=\frac{\left|D_{\sigma, \sigma^{*}} \cap K\right|}{\left|D_{\sigma, \sigma^{*}}\right|} \sum_{\substack{\mathfrak{s} \in \partial D_{\sigma, \sigma^{*}} \\ \mathfrak{s} \subset L}} \frac{|\mathfrak{s}|}{|\sigma|} \mathcal{F}_{D_{\sigma, \sigma^{*}, \mathfrak{s}}}-\frac{\left|D_{\sigma, \sigma^{*}} \cap L\right|}{\left|D_{\sigma, \sigma^{*}}\right|} \sum_{\substack{\mathfrak{s} \in \partial D_{\sigma, \sigma^{*}} \\ \mathfrak{s} \subset K}} \frac{|\mathfrak{s}|}{|\sigma|} \mathcal{F}_{D_{\sigma, \sigma^{*}, \mathfrak{s}}}
$$

These mass fluxes will be used to obtain a mass conservation equation on the primal mesh, but they will also enter into the definition of the momentum fluxes. For this purpose, we need to split

$$
\mathcal{F}_{K, \sigma}=\mathcal{F}_{K, \sigma}^{+}+\mathcal{F}_{K, \sigma}^{-}, \quad \pm \mathcal{F}_{K, \sigma}^{ \pm} \geqslant 0,
$$

since we wish to apply upwinding principles. A naive attempt would consist in performing the same construction starting from the original decomposition $\mathcal{F}_{D_{\sigma, \sigma^{*}, \mathfrak{s}}}=\mathcal{F}_{D_{\sigma, \sigma^{*}, \mathfrak{s}}}^{+}+\mathcal{F}_{D_{\sigma, \sigma^{*}, \mathfrak{s}}}^{-}$but there is no reason that the corresponding fluxes (13) on the interfaces of $K$ and $K^{*}$ preserve the sign property. Instead, we simply rearrange terms in (14)

$$
\begin{aligned}
& \mathcal{F}_{K, \sigma}=\underbrace{\frac{\left|D_{\sigma, \sigma^{*}} \cap K\right|}{\left|D_{\sigma, \sigma^{*}}\right|} \sum_{\substack{\mathfrak{s} \in \partial D_{\sigma, \sigma^{*}} \\
\mathfrak{s} \subset L}} \frac{|\mathfrak{s}|}{|\sigma|} \mathcal{F}_{D_{\sigma, \sigma^{*}, \mathfrak{s}}}^{+}-\frac{\left|D_{\sigma, \sigma^{*}} \cap L\right|}{\left|D_{\sigma, \sigma^{*}}\right|} \sum_{\substack{\mathfrak{s} \in \partial D_{\sigma, \sigma^{*}} \\
\mathfrak{s} \subset K}} \frac{|\mathfrak{s}|}{|\sigma|} \mathcal{F}_{D_{\sigma, \sigma^{*}, \mathfrak{s}}}}_{\geqslant 0} \\
& +\underbrace{\frac{\left|D_{\sigma, \sigma^{*}} \cap K\right|}{\left|D_{\sigma, \sigma^{*}}\right|} \sum_{\substack{\mathfrak{s} \in \partial D_{\sigma, \sigma^{*}} \\
\mathfrak{s} \subset L}} \frac{|\mathfrak{s}|}{|\sigma|} \mathcal{F}_{D_{\sigma, \sigma^{*}, \mathfrak{s}}}^{-}-\frac{\left|D_{\sigma, \sigma^{*}} \cap L\right|}{\left|D_{\sigma, \sigma^{*}}\right|} \sum_{\substack{\mathfrak{s} \in \partial D_{\sigma, \sigma^{*}} \\
\mathfrak{s} \subset K}} \frac{|\mathfrak{s}|}{|\sigma|} \mathcal{F}_{D_{\sigma, \sigma^{*}, \mathfrak{s}}^{+}}^{+}}_{\leqslant 0} .
\end{aligned}
$$

This is the definition we are going to use for $\mathcal{F}_{D_{\sigma, \sigma^{*}, \mathfrak{s}}^{+}}$and $\mathcal{F}_{D_{\sigma, \sigma^{*}, \mathfrak{s}}}^{-}$, namely

$$
\mathcal{F}_{K, \sigma}^{ \pm}=\frac{\left|D_{\sigma, \sigma^{*}} \cap K\right|}{\left|D_{\sigma, \sigma^{*}}\right|} \sum_{\substack{\mathfrak{s} \in \partial D_{\sigma, \sigma^{*}} \\ \mathfrak{s} \subset L}} \frac{|\mathfrak{s}|}{|\sigma|} \mathcal{F}_{D_{\sigma, \sigma^{*}, \mathfrak{s}}^{ \pm}}^{ \pm}-\frac{\left|D_{\sigma, \sigma^{*}} \cap L\right|}{\left|D_{\sigma, \sigma^{*}}\right|} \sum_{\substack{\mathfrak{s} \in \partial D_{\sigma, \sigma^{*}} \\ \mathfrak{s} \subset K}} \frac{|\mathfrak{s}|}{|\sigma|} \mathcal{F}_{D_{\sigma, \sigma^{*}, \mathfrak{s}}}^{\mp}
$$

Owing to the convexity assumption on the diamond cells, the diagonal $\sigma^{*}$ is an edge of the dual mesh, and we obtain similarly the following expression for the mass fluxes on the dual cells

$$
\mathcal{F}_{K^{*}, \sigma^{*}}=\mathcal{F}_{K^{*}, \sigma^{*}}^{+}+\mathcal{F}_{K^{*}, \sigma^{*}}^{-}
$$

with

$$
\mathcal{F}_{K^{*}, \sigma^{*}}^{ \pm}=\frac{\left|D_{\sigma, \sigma^{*}} \cap K^{*}\right|}{\left|D_{\sigma, \sigma^{*}}\right|} \sum_{\substack{\mathfrak{s} \in \partial D_{\sigma, \sigma^{*}} \\ \mathfrak{s} \subset L^{*}}} \frac{|\mathfrak{s}|}{\left|\sigma^{*}\right|} \mathcal{F}_{D_{\sigma, \sigma^{*}, \mathfrak{s}}^{ \pm}}^{ \pm} \frac{\left|D_{\sigma, \sigma^{*}} \cap L^{*}\right|}{\left|D_{\sigma, \sigma^{*}}\right|} \sum_{\substack{\mathfrak{s} \in \partial D_{\sigma, \sigma^{*}} \\ \mathfrak{s} \subset K^{*}}} \frac{|\mathfrak{s}|}{\left|\sigma^{*}\right|} \mathcal{F}_{D_{\sigma, \sigma^{*}, \mathfrak{s}}^{\mp}}^{\mp}
$$

What is crucial is the fact the fluxes are conservative: with $\sigma=K \mid L$ and $\sigma^{*}=K^{*} \mid L^{*}$,

$$
\mathcal{F}_{K, \sigma}=-\mathcal{F}_{L, \sigma} \quad \text { and } \quad \mathcal{F}_{K^{*}, \sigma^{*}}=-\mathcal{F}_{L^{*}, \sigma^{*}} .
$$


More specifically we have

$$
\mathcal{F}_{K, \sigma}^{ \pm}=-\mathcal{F}_{L, \sigma}^{\mp} \quad \text { and } \quad \mathcal{F}_{K^{*}, \sigma^{*}}^{ \pm}=-\mathcal{F}_{L^{*}, \sigma^{*}}^{\mp} .
$$

For interfaces $\sigma$ and $\sigma^{*}$ such that $D_{\sigma, \sigma^{*}} \in \mathfrak{D}_{\text {ext }}$, since $\mathcal{F}_{\sigma}$ was previously defined as the flux outgoing through $\sigma$, we naturally set

$$
\mathcal{F}_{K, \sigma}=\mathcal{F}_{\sigma} \quad \text { and } \quad \mathcal{F}_{K^{*}, \sigma}=\mathcal{F}_{\sigma} .
$$

with $K$ the unique cell in $\mathfrak{M}$ such that $D_{\sigma, \sigma^{*}} \subset K$ and $\sigma^{*}=K^{*} \mid L^{*}$. Moreover, denoting $\sigma=\left[x_{K^{*}}, x_{L^{*}}\right]$, we set $\mathcal{F}_{K^{*}, \sigma^{*}}=\mathcal{F}_{K^{*}, \sigma^{*}}^{+}+\mathcal{F}_{K^{*}, \sigma^{*}}^{-}$with

$$
\begin{aligned}
\left|\sigma^{*}\right| \mathcal{F}_{K^{*}, \sigma^{*}}^{ \pm}=\frac{\left|D_{\sigma, \sigma^{*}} \cap K^{*}\right|}{\left|D_{\sigma, \sigma^{*}}\right|}\left|\mathfrak{s}_{K L^{*}}\right| \mathcal{F}_{D_{\sigma, \sigma^{*}, \mathfrak{s}_{K L}}^{ \pm}} & -\frac{\left|D_{\sigma, \sigma^{*}} \cap L^{*}\right|}{\left|D_{\sigma, \sigma^{*}}\right|}\left|\mathfrak{s}_{K K^{*}}\right| \mathcal{F}_{D_{\sigma, \sigma^{*}, \mathfrak{s}_{K} K^{*}}^{\mp}} \\
+ & +\frac{\left|D_{\sigma, \sigma^{*}} \cap K^{*}\right|}{2\left|D_{\sigma, \sigma^{*}}\right|}|\sigma| \mathcal{F}_{\sigma}^{ \pm}-\frac{\left|D_{\sigma, \sigma^{*}} \cap L^{*}\right|}{2\left|D_{\sigma, \sigma^{*}}\right|}|\sigma| \mathcal{F}_{\sigma}^{\mp} .
\end{aligned}
$$

With the motivation of writing a conservative equation for the momentum $\rho \mathbf{u}$, we introduce averaged densities on $\mathfrak{T}$.

Definition 3.4. The averaged density on a cell $K$ of the primal mesh is defined by

$$
\rho_{K}=\sum_{D_{\sigma, \sigma^{*} \in \mathfrak{D}_{K}}} \frac{\left|D_{\sigma, \sigma^{*}} \cap K\right|}{|K|} \rho_{\sigma, \sigma^{*}} \text { for } K \in \mathfrak{M}
$$

and on a cell $K^{*}$ of the dual mesh, we set

$$
\rho_{K^{*}}=\sum_{D_{\sigma, \sigma^{*}} \in \mathfrak{D}_{K^{*}}} \frac{\left|D_{\sigma, \sigma^{*}} \cap K^{*}\right|}{\left|K^{*}\right|} \rho_{\sigma, \sigma^{*}} \text { for } K^{*} \in \mathfrak{M}^{*} \cup \partial \mathfrak{M}^{*} .
$$

With this definition at hand, considering either the primal or the dual mesh, the averaged densities $\rho_{K}$ and $\rho_{K^{*}}$ satisfy conservative equations, as observed in [21].

Proposition 3.5. The averaged densities $\rho_{K}, \rho_{K^{*}}$ satisfy the following conservative equations for any $K \in \mathfrak{M}$ and any $K^{*} \in \mathfrak{M}^{*} \cup \partial \mathfrak{M}^{*}$ :

$$
\begin{aligned}
& |K| \frac{\bar{\rho}_{K}-\rho_{K}}{\delta t}+\sum_{D_{\sigma, \sigma^{*} \in \mathfrak{D}_{K}}}|\sigma| \mathcal{F}_{K, \sigma}=0,
\end{aligned}
$$

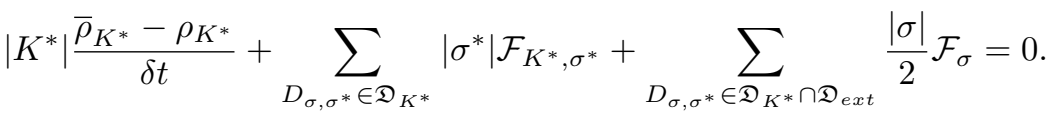

Proof. This is a consequence of the construction of the fluxes. Indeed, when considering $|K| \frac{\bar{\rho}_{K}-\rho_{K}}{\delta t}$, we are led to compute

$$
\begin{aligned}
& \sum_{D_{\sigma, \sigma^{*}} \in \mathfrak{D}_{K} \cap \mathfrak{D}_{i n t}}\left(\frac{\left|D_{\sigma, \sigma^{*}} \cap K\right|}{\left|D_{\sigma, \sigma^{*}}\right|} \sum_{\mathfrak{s} \in \partial D_{\sigma, \sigma^{*}}}|\mathfrak{s}| \mathcal{F}_{D_{\sigma, \sigma^{*}, \mathfrak{s}}}\right) \\
& +\sum_{D_{\sigma, \sigma^{*}} \in \mathfrak{D}_{K} \cap \mathfrak{D}_{\text {ext }}}\left(\frac{\left|D_{\sigma, \sigma^{*}} \cap K\right|}{\left|D_{\sigma, \sigma^{*}}\right|}\left[|\sigma| \mathcal{F}_{\sigma}+\sum_{\mathfrak{s} \in \partial D_{\sigma, \sigma^{*}} \backslash \partial \Omega}|\mathfrak{s}| \mathcal{F}_{D_{\sigma, \sigma^{*}, \mathfrak{s}}}\right]\right) .
\end{aligned}
$$

By definition and going back to Lemma 3.3, we get

$$
\begin{aligned}
\sum_{D_{\sigma, \sigma^{*}} \in \mathfrak{D}_{K} \cap \mathfrak{D}_{i n t}}\left(\frac{\left|D_{\sigma, \sigma^{*}} \cap K\right|}{\left|D_{\sigma, \sigma^{*}}\right|} \sum_{\mathfrak{s} \in \partial D_{\sigma, \sigma^{*}}}|\mathfrak{s}| \mathcal{F}_{D_{\sigma, \sigma^{*}, \mathfrak{s}}}\right) \\
=\sum_{D_{\sigma, \sigma^{*}} \in \mathfrak{D}_{K} \cap \mathfrak{D}_{i n t}}\left|D_{\sigma, \sigma^{*}} \cap K\right| \boldsymbol{\nabla} \cdot \omega_{D_{\sigma, \sigma^{*}}}=\sum_{D_{\sigma, \sigma^{*}} \in \mathfrak{D}_{K} \cap \mathfrak{D}_{i n t}} \int_{D_{\sigma, \sigma^{*}} \cap K} \boldsymbol{\nabla} \cdot \omega_{D_{\sigma, \sigma^{*}}} .
\end{aligned}
$$


By virtue of the divergence theorem, this becomes

$$
\begin{aligned}
& \sum_{D_{\sigma, \sigma^{*}} \in \mathfrak{D}_{K} \cap \mathfrak{D}_{i n t}}\left(\sum_{\mathfrak{s} \in \partial(D \cap K)} \int_{\mathfrak{s}} \omega_{D_{\sigma, \sigma^{*}}} \cdot \mathbf{n}_{\mathfrak{s}}\right) \\
= & \sum_{D_{\sigma, \sigma^{*}} \in \mathfrak{D}_{K} \cap \mathfrak{D}_{i n t}}\left(\int_{\sigma} \omega_{D_{\sigma, \sigma^{*}}} \cdot \mathbf{n}_{\sigma}+\sum_{\mathfrak{s} \in \partial D_{\sigma, \sigma^{*}}, s \subset K} \int_{\mathfrak{s}} \omega_{\left.D_{\sigma, \sigma^{*}} \cdot \mathbf{n}_{\mathfrak{s}}\right)}\left(|\sigma| \mathcal{F}_{K, \sigma}+\sum_{\mathfrak{s} \in \partial D_{\sigma, \sigma^{*}}, \mathfrak{s} \subset K}|\mathfrak{s}| \mathcal{F}_{D_{\sigma, \sigma^{*}, \mathfrak{s}}}\right)\right. \\
= & \sum_{D_{\sigma, \sigma^{*}} \in \mathfrak{D}_{K} \cap \mathfrak{D}_{i n t}}|\sigma| \mathcal{F}_{K, \sigma}+0 .
\end{aligned}
$$

A similar computation holds for the external cells and the dual cells. The result can be equally be checked by using the explicit formula (15) and (16), see [45].

\subsection{Momentum equation on the primal and dual cells}

We now turn to the definition of the momentum fluxes $\mathcal{G}_{K, \sigma}$ for the primal cells and $\mathcal{G}_{K^{*}, \sigma^{*}}$ for the dual cells. We first consider the case of interfaces $\sigma \not \subset \partial \Omega$. In this case, for the primal cells we set

$$
\mathcal{G}_{K, \sigma}=\mathcal{F}_{K, \sigma}^{+} \mathbf{u}_{K}+\mathcal{F}_{K, \sigma}^{-} \mathbf{u}_{L}
$$

Namely, for defining the momentum fluxes we use the mass fluxes $\mathcal{F}_{K, \sigma}^{ \pm}$given by (15). For the dual cells, a similar formula based on (16)

$$
\mathcal{G}_{K^{*}, \sigma^{*}}=\mathcal{F}_{K^{*}, \sigma^{*}}^{+} \mathbf{u}_{K^{*}}+\mathcal{F}_{K^{*}, \sigma^{*}}^{-} \mathbf{u}_{L^{*}} .
$$

applies directly: the convexity assumption of the diamond cells implies that $\sigma^{*}$ is an edge for the dual cell and is included in $D_{\sigma, \sigma^{*}}$. Note that the momentum fluxes are conservative as a consequence of (17). For the boundary conditions, that is for $\sigma \subset \partial \Omega$, we define:

$$
\mathcal{G}_{K, \sigma}=\mathcal{F}_{\sigma}^{+} \mathbf{u}_{K}+\mathcal{F}_{\sigma}^{-} \mathbf{u}_{\sigma} \text { and } \mathcal{G}_{K^{*}, \sigma}=\mathcal{F}_{\sigma}^{+} \mathbf{u}_{K^{*}}+\mathcal{F}_{\sigma}^{-} \mathbf{u}_{\sigma} .
$$

Remark 3.6. For the fluxes $\mathcal{G}_{K, \sigma}$, the formula (19) is valid also for $\sigma \subset \partial \Omega$ if we use the convention $\mathbf{u}_{L}=\mathbf{u}_{\sigma}$ in this case.

The momentum equation also requires to introduce a discrete pressure gradient. It is obtained by mimicking the formula

$$
\int_{X} \nabla p=\int_{\partial X} p \mathbf{n}
$$

Definition 3.7. The discrete pressure gradient $\nabla_{\boldsymbol{d}} p$ is defined on $\mathfrak{T}$ by

$$
\begin{array}{ll}
\left(\boldsymbol{\nabla}_{\boldsymbol{d}} p\right)_{K}=\frac{1}{|K|} \sum_{D_{\sigma, \sigma^{*} \in \mathfrak{D}_{K}}|\sigma| p_{\sigma, \sigma^{*}} \mathbf{n}_{K, \sigma},} & \text { for } K \in \mathfrak{M}, \\
\left(\boldsymbol{\nabla}_{\boldsymbol{d}} p\right)_{K^{*}}=\frac{1}{\left|K^{*}\right|} \sum_{D_{\sigma, \sigma^{*} \in \mathfrak{D}_{K^{*}}}|\sigma| p_{\sigma, \sigma^{*}} \mathbf{n}_{K^{*}, \sigma^{*}},} & \text { for } K^{*} \in \mathfrak{M}^{*}, \\
\left(\boldsymbol{\nabla}_{\boldsymbol{d}} p\right)_{K^{*}}=\frac{1}{\left|K^{*}\right|} \sum_{D_{\sigma, \sigma^{*}} \in \mathfrak{D}_{K^{*}} \cap \mathfrak{D}_{i n t}}\left|\sigma^{*}\right| p_{\sigma, \sigma^{*}} \mathbf{n}_{K^{*}, \sigma^{*}}+\sum_{D_{\sigma, \sigma^{*}} \in \mathfrak{D}_{K^{*}} \cap \mathfrak{D}_{i n t}} \frac{|\sigma|}{2} p_{\sigma, \sigma^{*}} \mathbf{n}_{K, \sigma}, & \text { for } K^{*} \in \partial \mathfrak{M}^{*} .
\end{array}
$$


The discrete momentum equation is given for $K \in \mathfrak{T}$ by

$$
\begin{aligned}
& \frac{\bar{\rho}_{K} \overline{\mathbf{u}}_{K}-\rho_{K} \mathbf{u}_{K}}{\delta t}+\frac{1}{|K|} \sum_{D_{\sigma, \sigma^{*} \in \mathfrak{D}_{K}}}|\sigma| \mathcal{G}_{K, \sigma}+\left(\boldsymbol{\nabla}_{\boldsymbol{d}} p\right)_{K}=0, \\
& \frac{\bar{\rho}_{K^{*}} \overline{\mathbf{u}}_{K^{*}}-\rho_{K^{*}} \mathbf{u}_{K^{*}}}{\delta t}+\frac{1}{\left|K^{*}\right|} \sum_{D_{\sigma, \sigma^{*}} \in \mathcal{D}_{K^{*}}}\left|\sigma^{*}\right| \mathcal{G}_{K^{*}, \sigma^{*}} \\
& +\frac{1}{\left|K^{*}\right|} \sum_{D_{\sigma, \sigma^{*}} \in \mathfrak{D}_{K^{*}} \cap \mathfrak{D}_{\text {ext }}} \frac{|\sigma|}{2} \mathcal{G}_{K^{*}, \sigma}+\left(\nabla_{\boldsymbol{d}} p\right)_{K^{*}}=0,
\end{aligned}
$$

with $\rho_{K}$ and $\rho_{K^{*}}$ given by Definition 3.4, fluxes defined in (19), (20) and pressure gradients in Definition 3.7.

\subsection{Internal energy balance on the diamond mesh}

As explained in Section 3, at the continuous level, considering smooth enough functions, the internal energy equation (2) is deduced from the kinetic energy balance, which is itself obtained by multiplying the momentum equation by $\mathbf{u}$. At the discrete level, multiplying the discrete momentum equation by $\mathbf{u}_{K}$ or $\mathbf{u}_{K^{*}}$, whatever the considered mesh, introduces a remainder term that has to be taken into account to write the discrete internal energy equation [30,33]. To this end, let us introduce kinetic fluxes $\mathcal{K}_{K, \sigma}$ from primal cells and $\mathcal{K}_{K^{*}, \sigma^{*}}, \mathcal{K}_{K^{*}, \sigma}$ from dual cells. For $\sigma \not \subset \partial \Omega$, we set

$$
\mathcal{K}_{K, \sigma}=\mathcal{F}_{K, \sigma}^{+} \frac{\left\|\mathbf{u}_{K}\right\|^{2}}{2}+\mathcal{F}_{K, \sigma}^{-} \frac{\left\|\mathbf{u}_{L}\right\|^{2}}{2}, \quad \text { and } \quad \mathcal{K}_{K^{*}, \sigma^{*}}=\mathcal{F}_{K^{*}, \sigma^{*}}^{+} \frac{\left\|\mathbf{u}_{K^{*}}\right\|^{2}}{2}+\mathcal{F}_{K^{*}, \sigma^{*}}^{-} \frac{\left\|\mathbf{u}_{L^{*}}\right\|^{2}}{2} .
$$

These fluxes are conservative $\left(\mathcal{K}_{K, \sigma}=-\mathcal{K}_{L, \sigma}\right.$, and so on...) as a consequence of (17). For the boundary conditions, that is $\sigma \subset \partial \Omega$, we set

$$
\mathcal{K}_{K, \sigma}=\mathcal{F}_{\sigma}^{+} \frac{\left\|\mathbf{u}_{K}\right\|^{2}}{2}+\mathcal{F}_{\sigma}^{-} \frac{\left\|\mathbf{u}_{\sigma}\right\|^{2}}{2} \text { and } \quad \mathcal{K}_{K^{*}, \sigma}=\mathcal{F}_{\sigma}^{+} \frac{\left\|\mathbf{u}_{K^{*}}\right\|^{2}}{2}+\mathcal{F}_{\sigma}^{-} \frac{\left\|\mathbf{u}_{\sigma}\right\|^{2}}{2} \text {. }
$$

Remark 3.8. Note that, as for momentum fluxes (see Remark 3.6), formula (22) for fluxes $\mathcal{K}_{K, \sigma}$, is valid also for $\sigma \subset \partial \Omega$ if we use the convention $\mathbf{u}_{L}=\mathbf{u}_{\sigma}$ in this case.

Definition 3.9. For $K \in \mathfrak{M}$ we set

$$
\mathcal{R}_{K}=\frac{\bar{\rho}_{K}}{2 \delta t}\left\|\overline{\mathbf{u}}_{K}-\mathbf{u}_{K}\right\|^{2}+\frac{1}{|K|} \sum_{D_{\sigma, \sigma^{*} \in \mathfrak{D}_{K}}}|\sigma| \mathcal{F}_{K, \sigma}^{-}\left(\frac{\left\|\overline{\mathbf{u}}_{K}-\mathbf{u}_{K}\right\|^{2}}{2}-\frac{\left\|\overline{\mathbf{u}}_{K}-\mathbf{u}_{L}\right\|^{2}}{2}\right),
$$

with the convention that $\mathbf{u}_{L}=\mathbf{u}_{\sigma}$ when $\sigma \subset \partial \Omega$.

For $K^{*} \in \mathfrak{M}^{*} \cup \partial \mathfrak{M}^{*}$ we set

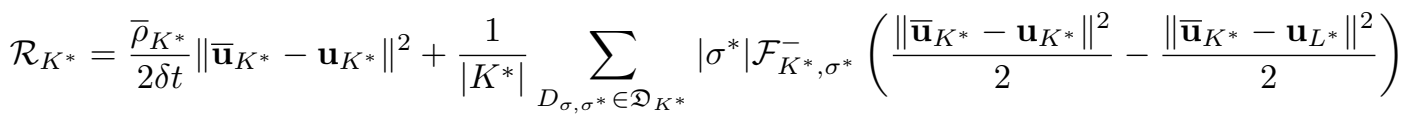

$$
\begin{aligned}
& +\frac{1}{\left|K^{*}\right|} \sum_{D_{\sigma, \sigma^{*}} \in \mathfrak{D}_{K^{*}} \cap \mathfrak{D}_{\text {ext }}} \frac{|\sigma|}{2} \mathcal{F}_{\sigma}^{-}\left(\frac{\left\|\overline{\mathbf{u}}_{K^{*}}-\mathbf{u}_{K^{*}}\right\|^{2}}{2}-\frac{\left\|\overline{\mathbf{u}}_{K^{*}}-\mathbf{u}_{\sigma}\right\|^{2}}{2}\right) .
\end{aligned}
$$

Lemma 3.10. The discrete balance of kinetic energy is given for $K \in \mathfrak{M}$ and $K^{*} \in \mathfrak{M}^{*} \cup \partial \mathfrak{M}^{*}$ by

$$
\frac{\bar{\rho}_{K} \frac{\left\|\overline{\mathbf{u}}_{K}\right\|^{2}}{2}-\rho_{K} \frac{\left\|\mathbf{u}_{K}\right\|^{2}}{2}}{\delta t}+\frac{1}{|K|} \sum_{D_{\sigma, \sigma^{*} \in \mathfrak{D}_{K}}}|\sigma| \mathcal{K}_{K, \sigma}+\left(\nabla_{d} p\right)_{K} \cdot \overline{\mathbf{u}}_{K}=-\mathcal{R}_{K} .
$$




$$
\begin{aligned}
\frac{\bar{\rho}_{K^{*}} \frac{\left\|\overline{\mathbf{u}}_{K^{*}}\right\|^{2}}{2}-\rho_{K^{*}}}{\delta t} & \frac{\left\|\mathbf{u}_{K^{*}}\right\|^{2}}{2} \\
& +\frac{1}{\left|K^{*}\right|} \sum_{D_{\sigma, \sigma} \in \mathfrak{D}_{K^{*}}}\left|\sigma^{*}\right| \mathcal{K}_{K^{*}, \sigma^{*}} \\
& +\frac{1}{\left|K^{*}\right|} \sum_{D_{\sigma, \sigma^{*}} \in \mathfrak{D}_{K^{*}} \cap \mathfrak{D}_{\text {ext }}} \frac{|\sigma|}{2} \mathcal{K}_{K^{*}, \sigma}+\left(\nabla_{\boldsymbol{d}} p\right)_{K^{*}} \cdot \overline{\mathbf{u}}_{K^{*}}=-\mathcal{R}_{K^{*}} .
\end{aligned}
$$

Proof. For $X \in \mathfrak{T}$, we multiply by $\overline{\mathbf{u}}_{X}$ the momentum equation (21) and use the averaged mass equation in Proposition (3.5).

a) Let $K \in \mathfrak{M}$. In what follows, we use the convention that $\mathbf{u}_{L}=\mathbf{u}_{\sigma}$ when the edge $\sigma \subset \partial \Omega$; so that the expressions (19) and (22) are valid for $\sigma \subset \partial \Omega$ too (see Remarks 3.6 and 3.8). We start by remarking that

$$
\begin{aligned}
\frac{\bar{\rho}_{K} \overline{\mathbf{u}}_{K}-\rho_{K} \mathbf{u}_{K}}{\delta t} \cdot \overline{\mathbf{u}}_{K}=\frac{1}{\delta t}\left(\bar{\rho}_{K} \frac{\left\|\overline{\mathbf{u}}_{K}\right\|^{2}}{2}-\rho_{K} \frac{\left\|\mathbf{u}_{K}\right\|^{2}}{2}\right. & \left.+\frac{\bar{\rho}_{K}}{2}\left\|\overline{\mathbf{u}}_{K}-\mathbf{u}_{K}\right\|^{2}\right) \\
& -\frac{\bar{\rho}_{K}-\rho_{K}}{\delta t}\left(\frac{\left\|\mathbf{u}_{K}\right\|^{2}}{2}-\mathbf{u}_{K} \cdot \overline{\mathbf{u}}_{K}\right) .
\end{aligned}
$$

Thus using the average mass balance stated in Proposition (3.5) we get

$$
\begin{aligned}
\frac{\bar{\rho}_{K} \overline{\mathbf{u}}_{K}-\rho_{K} \mathbf{u}_{K}}{\delta t} \cdot \overline{\mathbf{u}}_{K}=\frac{1}{\delta t}\left(\bar{\rho}_{K} \frac{\left\|\overline{\mathbf{u}}_{K}\right\|^{2}}{2}-\rho_{K}\right. & \left.\frac{\left\|\mathbf{u}_{K}\right\|^{2}}{2}+\frac{\bar{\rho}_{K}}{2}\left\|\overline{\mathbf{u}}_{K}-\mathbf{u}_{K}\right\|^{2}\right) \\
& +\frac{1}{|K|} \sum_{D_{\sigma, \sigma^{*} \in \mathfrak{D}_{K}}|\sigma| \mathcal{F}_{K, \sigma}\left(\frac{\left\|\mathbf{u}_{K}\right\|^{2}}{2}-\mathbf{u}_{K} \cdot \overline{\mathbf{u}}_{K}\right) .}
\end{aligned}
$$

Next, using the notation $\mathcal{F}^{|\cdot|}=\mathcal{F}^{+}-\mathcal{F}^{-}$and bearing in mind that $\mathcal{F}=\mathcal{F}^{+}+\mathcal{F}^{-}$, we get

$$
\mathcal{G}_{K, \sigma}=\frac{\mathcal{F}_{K, \sigma}^{|\cdot|}+\mathcal{F}_{K, \sigma}}{2} \mathbf{u}_{K}+\frac{\mathcal{F}_{K, \sigma}-\mathcal{F}_{K, \sigma}^{|\cdot|}}{2} \mathbf{u}_{L}=\mathcal{F}_{K, \sigma} \frac{\mathbf{u}_{K}+\mathbf{u}_{L}}{2}+\mathcal{F}_{K, \sigma}^{|.|} \frac{\mathbf{u}_{K}-\mathbf{u}_{L}}{2}
$$

Hence, the momentum equation multiplied by $\overline{\mathbf{u}}_{K}$ becomes

$$
\frac{\bar{\rho}_{K} \frac{\left\|\overline{\mathbf{u}}_{K}\right\|^{2}}{2}-\rho_{K} \frac{\left\|\mathbf{u}_{K}\right\|^{2}}{2}}{\delta t}+\frac{\bar{\rho}_{K}}{2 \delta t}\left\|\overline{\mathbf{u}}_{K}-\mathbf{u}_{K}\right\|^{2}+\left(\nabla_{\boldsymbol{d}} p\right)_{K} \cdot \overline{\mathbf{u}}_{K}+\frac{1}{|K|} \sum_{D_{\sigma, \sigma *} \in \mathfrak{D}_{K}}|\sigma| B_{K, \sigma}=0
$$

where

$$
\begin{aligned}
B_{K, \sigma} & =\mathcal{F}_{K, \sigma}\left(\frac{\left\|\mathbf{u}_{K}\right\|^{2}}{2}-\frac{\mathbf{u}_{K}-\mathbf{u}_{L}}{2} \cdot \overline{\mathbf{u}}_{K}\right)+\mathcal{F}_{K, \sigma}^{|\cdot|} \frac{\mathbf{u}_{K}-\mathbf{u}_{L}}{2} \cdot \overline{\mathbf{u}}_{K} \\
& =\left(\mathcal{F}_{K, \sigma}^{+} \frac{\left\|\mathbf{u}_{K}\right\|^{2}}{2}+\mathcal{F}_{K, \sigma}^{-} \frac{\left\|\mathbf{u}_{L}\right\|^{2}}{2}\right)+\mathcal{F}_{K, \sigma}^{-}\left(\frac{\left\|\mathbf{u}_{K}\right\|^{2}}{2}-\frac{\left\|\mathbf{u}_{L}\right\|^{2}}{2}-\left(\mathbf{u}_{K}-\mathbf{u}_{L}\right) \cdot \overline{\mathbf{u}}_{K}\right) .
\end{aligned}
$$

With definition (22) we are left with

$$
B_{K, \sigma}=\mathcal{K}_{K, \sigma}+\mathcal{F}_{K, \sigma}^{-}\left(\frac{\left\|\overline{\mathbf{u}}_{K}-\mathbf{u}_{K}\right\|^{2}}{2}-\frac{\left\|\overline{\mathbf{u}}_{K}-\mathbf{u}_{L}\right\|^{2}}{2}\right),
$$

and thus we obtain (23).

b) For $K^{*} \in \mathfrak{M}^{*} \cup \partial \mathfrak{M}^{*}$, as previously, we first remark that

$$
\begin{aligned}
\frac{\bar{\rho}_{K^{*}} \overline{\mathbf{u}}_{K^{*}}-\rho_{K^{*}} \mathbf{u}_{K^{*}}}{\delta t} \cdot \overline{\mathbf{u}}_{K^{*}}=\frac{1}{\delta t}\left(\bar{\rho}_{K^{*}} \frac{\left\|\overline{\mathbf{u}}_{K^{*}}\right\|^{2}}{2}-\rho_{K^{*}} \frac{\left\|\mathbf{u}_{K^{*}}\right\|^{2}}{2}+\frac{\bar{\rho}_{K^{*}}}{2}\left\|\overline{\mathbf{u}}_{K^{*}}-\mathbf{u}_{K^{*}}\right\|^{2}\right) \\
-\frac{\bar{\rho}_{K^{*}}-\rho_{K^{*}}}{\delta t}\left(\frac{\left\|\mathbf{u}_{K^{*}}\right\|^{2}}{2}-\mathbf{u}_{K^{*}} \cdot \overline{\mathbf{u}}_{K^{*}}\right) .
\end{aligned}
$$


Thus using the average mass balance stated in Proposition (3.5) we get

$$
\begin{aligned}
& \frac{\bar{\rho}_{K^{*}} \overline{\mathbf{u}}_{K^{*}}-\rho_{K^{*}} \mathbf{u}_{K^{*}}}{\delta t} \cdot \overline{\mathbf{u}}_{K^{*}}=\frac{1}{\delta t}\left(\bar{\rho}_{K^{*}} \frac{\left\|\overline{\mathbf{u}}_{K^{*}}\right\|^{2}}{2}-\rho_{K^{*}} \frac{\left\|\mathbf{u}_{K^{*}}\right\|^{2}}{2}+\frac{\bar{\rho}_{K^{*}}}{2}\left\|\overline{\mathbf{u}}_{K^{*}}-\mathbf{u}_{K^{*}}\right\|^{2}\right) \\
& +\frac{1}{\left|K^{*}\right|} \sum_{D_{\sigma, \sigma^{*}} \in \mathfrak{D}_{K^{*}}}\left|\sigma^{*}\right| \mathcal{F}_{K^{*}, \sigma^{*}}\left(\frac{\left\|\mathbf{u}_{K^{*}}\right\|^{2}}{2}-\mathbf{u}_{K^{*}} \cdot \overline{\mathbf{u}}_{K^{*}}\right) \\
& +\frac{1}{\left|K^{*}\right|} \sum_{D_{\sigma, \sigma^{*}} \in \mathfrak{D}_{K^{*}} \cap \mathfrak{D}_{e x t}} \frac{|\sigma|}{2} \mathcal{F}_{K^{*}, \sigma}\left(\frac{\left\|\mathbf{u}_{K^{*}}\right\|^{2}}{2}-\mathbf{u}_{K^{*}} \cdot \overline{\mathbf{u}}_{K^{*}}\right) .
\end{aligned}
$$

The momentum equation multiplied by $\overline{\mathbf{u}}_{K^{*}}$ becomes

$$
\begin{aligned}
& \frac{\bar{\rho}_{K^{*}} \frac{\left\|\overline{\mathbf{u}}_{K^{*}}\right\|^{2}}{2}-\rho_{K^{*}} \frac{\left\|\mathbf{u}_{K^{*}}\right\|^{2}}{2}}{\delta t}+\frac{\bar{\rho}_{K^{*}}}{2 \delta t}\left\|\overline{\mathbf{u}}_{K^{*}}-\mathbf{u}_{K^{*}}\right\|^{2}+\left(\nabla_{\boldsymbol{d}} p\right)_{K^{*}} \cdot \overline{\mathbf{u}}_{K^{*}} \\
& +\frac{1}{\left|K^{*}\right|}\left(\sum_{D_{\sigma, \sigma^{*}} \in \mathfrak{D}_{K^{*}}}\left|\sigma^{*}\right| \mathcal{F}_{K^{*}, \sigma^{*}}+\sum_{D_{\sigma, \sigma^{*}} \in \mathfrak{D}_{K^{*}} \cap \mathfrak{D}_{\text {ext }}} \frac{|\sigma|}{2} \mathcal{F}_{\sigma}\right)\left(\frac{\left\|\mathbf{u}_{K^{*}}\right\|^{2}}{2}-\mathbf{u}_{K^{*}} \cdot \overline{\mathbf{u}}_{K^{*}}\right) \\
& +\frac{1}{\left|K^{*}\right|}\left(\sum_{D_{\sigma, \sigma^{*}} \in \mathfrak{D}_{K^{*}}}\left|\sigma^{*}\right| \mathcal{G}_{K^{*}, \sigma^{*}}+\sum_{D_{\sigma, \sigma^{*}} \in \mathfrak{D}_{K^{*}} \cap \mathfrak{D}_{\text {ext }}} \frac{|\sigma|}{2} \mathcal{G}_{K^{*}, \sigma}\right) \cdot \overline{\mathbf{u}}_{K^{*}}=0 .
\end{aligned}
$$

We obtain (24) by remarking that, as in the first part of the proof, we have for all $D_{\sigma, \sigma^{*}} \in \mathfrak{D}_{K^{*}}$

$$
\begin{aligned}
\mathcal{F}_{K^{*}, \sigma^{*}}\left(\frac{\left\|\mathbf{u}_{K^{*}}\right\|^{2}}{2}-\mathbf{u}_{K^{*}} \cdot \overline{\mathbf{u}}_{K^{*}}\right)+\mathcal{G}_{K^{*}, \sigma^{*}} \cdot \overline{\mathbf{u}}_{K^{*}} \\
=\mathcal{K}_{K^{*}, \sigma^{*}}+\mathcal{F}_{K^{*}, \sigma^{*}}^{-}\left(\frac{\left\|\overline{\mathbf{u}}_{K^{*}}-\mathbf{u}_{K^{*}}\right\|^{2}}{2}-\frac{\left\|\overline{\mathbf{u}}_{K^{*}}-\mathbf{u}_{L^{*}}\right\|^{2}}{2}\right) .
\end{aligned}
$$

and similarly, we also get for all $D_{\sigma, \sigma^{*}} \in \mathfrak{D}_{K^{*}} \cap \mathfrak{D}_{\text {ext }}$ that

$$
\begin{aligned}
\mathcal{F}_{\sigma}\left(\frac{\left\|\mathbf{u}_{K^{*}}\right\|^{2}}{2}-\mathbf{u}_{K^{*}} \cdot \overline{\mathbf{u}}_{K^{*}}\right) & +\mathcal{G}_{K^{*}, \sigma} \cdot \overline{\mathbf{u}}_{K^{*}} \\
= & \mathcal{K}_{K^{*}, \sigma}+\mathcal{F}_{\sigma}^{-}\left(\frac{\left\|\overline{\mathbf{u}}_{K^{*}}-\mathbf{u}_{K^{*}}\right\|^{2}}{2}-\frac{\left\|\overline{\mathbf{u}}_{K^{*}}-\mathbf{u}_{\sigma}\right\|^{2}}{2}\right) .
\end{aligned}
$$

We remind the reader that the kinetic energy equation does not have to be solved since we already know the velocity on the primal and dual meshes from the momentum equation. This computation only aims at defining the remainder terms $\mathcal{R}_{K}$ and $\mathcal{R}_{K^{*}}$ that will be used in the equation for updating the internal energy. Before this, we need to introduce a discrete divergence operator, naturally inspired from

$$
\int_{X} \boldsymbol{\nabla} \cdot \mathbf{u}=\int_{\partial X} \mathbf{u} \cdot \mathbf{n}
$$

Definition 3.11. The discrete divergence operator on a cell $D_{\sigma, \sigma^{*}} \in \mathfrak{D}$ is defined as

$$
\left(\nabla_{\boldsymbol{d}} \cdot \mathbf{u}\right)_{\sigma, \sigma^{*}}=\frac{1}{\left|D_{\sigma, \sigma^{*}}\right|} \sum_{\mathfrak{s} \in \partial D_{\sigma, \sigma^{*}}}|\mathfrak{s}| u_{D_{\sigma, \sigma^{*}, \mathfrak{s}}}
$$

when $D_{\sigma, \sigma^{*}} \in \mathfrak{D}_{\text {int }}$, while for $D_{\sigma, \sigma^{*}} \in \mathfrak{D}_{\text {ext }}$, we set

$$
\left(\boldsymbol{\nabla}_{\boldsymbol{d}} \cdot \mathbf{u}\right)_{\sigma, \sigma^{*}}=\frac{1}{\left|D_{\sigma, \sigma^{*}}\right|} \sum_{\mathfrak{s} \in \partial D_{\sigma, \sigma^{*}} \backslash \partial \Omega}|\mathfrak{s}| u_{D_{\sigma, \sigma^{*}, \mathfrak{s}}}+\frac{|\sigma|}{2\left|D_{\sigma, \sigma^{*}}\right|}\left(\mathbf{u}_{\sigma}+\frac{\mathbf{u}_{K^{*}}+\mathbf{u}_{L^{*}}}{2}\right) \cdot \mathbf{n}_{K, \sigma} .
$$


The following statement gives an equivalent formulation of the discrete divergence; it will be useful to study the stability of the scheme.

Lemma 3.12. The discrete divergence operator on a cell $D_{\sigma, \sigma^{*}} \in \mathfrak{D}$ recasts as

$$
\begin{array}{ll}
\left(\boldsymbol{\nabla}_{\boldsymbol{d}} \cdot \mathbf{u}\right)_{\sigma, \sigma^{*}}=\frac{1}{2 \mid D_{\sigma, \sigma^{*}}}\left(|\sigma|\left(\mathbf{u}_{L}-\mathbf{u}_{K}\right) \cdot \mathbf{n}_{K, \sigma}+\left|\sigma^{*}\right|\left(\mathbf{u}_{L^{*}}-\mathbf{u}_{K^{*}}\right) \cdot \mathbf{n}_{K^{*}, \sigma^{*}}\right), \quad \forall D_{\sigma, \sigma^{*}} \in \mathfrak{D}_{\text {int }} . \\
\left(\boldsymbol{\nabla}_{\boldsymbol{d}} \cdot \mathbf{u}\right)_{\sigma, \sigma^{*}}=\frac{1}{2\left|D_{\sigma, \sigma^{*}}\right|}\left(|\sigma|\left(\mathbf{u}_{\sigma}-\mathbf{u}_{K}\right) \cdot \mathbf{n}_{K, \sigma}+\left|\sigma^{*}\right|\left(\mathbf{u}_{L^{*}}-\mathbf{u}_{K^{*}}\right) \cdot \mathbf{n}_{K^{*}, \sigma^{*}}\right), \quad \forall D_{\sigma, \sigma^{*}} \in \mathfrak{D}_{\text {ext }} .
\end{array}
$$

Proof. Let us first assume that $D_{\sigma, \sigma^{*}} \in \mathfrak{D}_{\text {int }}$. Using the definition of $u_{D_{\sigma, \sigma^{*}, \mathfrak{s}}}$ and of $\left(\nabla_{\boldsymbol{d}} \cdot \mathbf{u}\right)_{\sigma, \sigma^{*}}$, we have

$$
\begin{aligned}
\sum_{\mathfrak{s} \in \partial D_{\sigma, \sigma^{*}}}|\mathfrak{s}| u_{D_{\sigma, \sigma^{*}, \mathfrak{s}}} & =\frac{\mathbf{u}_{K}}{2} \cdot\left(\left|\mathfrak{s}_{K K^{*}}\right| \mathbf{n}_{\sigma, \mathfrak{s}_{K K^{*}}}+\left|\mathfrak{s}_{K L^{*}}\right| \mathbf{n}_{\sigma, \mathfrak{s}_{K L^{*}}}\right)+\frac{\mathbf{u}_{K^{*}}}{2} \cdot\left(\left|\mathfrak{s}_{K K^{*}}\right| \mathbf{n}_{\sigma, \mathfrak{s}_{K K^{*}}}+\left|\mathfrak{s}_{L K^{*}}\right| \mathbf{n}_{\sigma, \mathfrak{s}_{L K^{*}}}\right) \\
& +\frac{\mathbf{u}_{L}}{2} \cdot\left(\left|\mathfrak{s}_{L K^{*}}\right| \mathbf{n}_{\sigma, \mathfrak{s}_{L K^{*}}}+\left|\mathfrak{s}_{L L^{*}}\right| \mathbf{n}_{\sigma, \mathfrak{s}_{L L^{*}}}\right)+\frac{\mathbf{u}_{L^{*}}}{2} \cdot\left(\left|\mathfrak{s}_{K L^{*}}\right| \mathbf{n}_{\sigma, \mathfrak{s}_{K L^{*}}}+\left|\mathfrak{s}_{L L^{*}}\right| \mathbf{n}_{\sigma, \mathfrak{s}_{L L^{*}}}\right)
\end{aligned}
$$

By using Lemma 2.4, it follows that

$$
\sum_{\mathfrak{s} \in \partial D_{\sigma, \sigma^{*}}}|\mathfrak{s}| u_{D_{\sigma, \sigma^{*}, \mathfrak{s}}}=-\frac{\mathbf{u}_{K}}{2} \cdot|\sigma| \mathbf{n}_{K, \sigma}-\frac{\mathbf{u}_{K^{*}}}{2} \cdot\left|\sigma^{*}\right| \mathbf{n}_{K^{*}, \sigma^{*}}-\frac{\mathbf{u}_{L}}{2} \cdot|\sigma| \mathbf{n}_{L, \sigma}-\frac{\mathbf{u}_{L^{*}}}{2} \cdot\left|\sigma^{*}\right| \mathbf{n}_{L^{*}, \sigma^{*}}
$$

We conclude by using $-\mathbf{n}_{L, \sigma}=\mathbf{n}_{K, \sigma}$ and $-\mathbf{n}_{L^{*}, \sigma^{*}}=\mathbf{n}_{K^{*}, \sigma^{*}}$.

The proof for $D_{\sigma, \sigma^{*}} \in \mathfrak{D}_{\text {ext }}$ follows exactly the same lines and is left to the reader.

For the discretization of the internal energy equation, we define the following numerical fluxes, for all $D_{\sigma, \sigma^{*}} \in \mathfrak{D}_{\text {int }}$ and $\mathfrak{s}=D_{\sigma, \sigma^{*}} \mid D_{\sigma^{\prime}, \sigma^{*}} \in \partial D_{\sigma, \sigma^{*}}$,

$$
\mathcal{E}_{D_{\sigma, \sigma^{*}, \mathfrak{s}}}=e_{\sigma, \sigma^{*}} \mathcal{F}_{D_{\sigma, \sigma^{*}, \mathfrak{s}}^{+}}^{+}+e_{\sigma^{\prime}, \sigma^{*}} \mathcal{F}_{D_{\sigma, \sigma^{*}, \mathfrak{s}}}^{-}
$$

We observe that the fluxes $\mathcal{E}_{D_{\sigma, \sigma^{*}, \mathfrak{s}}}$ are conservative by definition. For $D_{\sigma, \sigma^{*}} \in \mathfrak{D}_{\text {ext }}$, we have to define the outgoing flux $\mathcal{E}_{\sigma}$ through the primal egde $\sigma \subset \partial D_{\sigma, \sigma^{*}} \cap \partial \Omega$. We take

$$
\mathcal{E}_{\sigma}=e_{\sigma, \sigma^{*}} \mathcal{F}_{\sigma}^{+}+e_{\sigma} \mathcal{F}_{\sigma}^{-}
$$

Finally, we also give the definition of a remainder term $\mathcal{R}_{\sigma, \sigma^{*}}$ on the diamond cell, which is based on the remainder term $\mathcal{R}_{K}$ and $\mathcal{R}_{K^{*}}$ given in Definition 3.9,

$$
\mathcal{R}_{\sigma, \sigma^{*}}=\frac{\left|D_{\sigma, \sigma^{*}} \cap K\right| \mathcal{R}_{K}+\left|D_{\sigma, \sigma^{*}} \cap L\right| \mathcal{R}_{L}+\left|D_{\sigma, \sigma^{*}} \cap K^{*}\right| \mathcal{R}_{K^{*}}+\left|D_{\sigma, \sigma^{*}} \cap L^{*}\right| \mathcal{R}_{L^{*}}}{2\left|D_{\sigma, \sigma^{*}}\right|},
$$

with the convention that $\mathcal{R}_{L}=0$ if $D_{\sigma, \sigma^{*}} \in \mathfrak{D}_{\text {ext }}$. This definition comes from the derivation of the local conservation of the total energy that will be discussed in the forthcoming Section 4. The remainder $\mathcal{R}_{\sigma, \sigma^{*}}$ is defined so that it exactly balances the kinetic energy contributions that will appear when summing the internal energy equation and the kinetic energy equations.

The discrete internal energy equation is given by

$$
\begin{aligned}
& \frac{\bar{\rho}_{\sigma, \sigma^{*}} \bar{e}_{\sigma, \sigma^{*}}-\rho_{\sigma, \sigma^{*}} e_{\sigma, \sigma^{*}}}{\delta t}+\frac{1}{\left|D_{\sigma, \sigma^{*}}\right|} \sum_{\mathfrak{s} \in \partial D_{\sigma, \sigma^{*}}}|\mathfrak{s}| \mathcal{E}_{D_{\sigma, \sigma^{*}, \mathfrak{s}}} \\
&+p_{\sigma, \sigma^{*}}\left(\boldsymbol{\nabla}_{\boldsymbol{d}} \cdot \overline{\mathbf{u}}\right)_{\sigma, \sigma^{*}}=\mathcal{R}_{\sigma, \sigma^{*}}, \quad \forall D_{\sigma, \sigma^{*}} \in \mathfrak{D}_{\text {int }} \\
& \frac{\bar{\rho}_{\sigma, \sigma^{*}} \bar{e}_{\sigma, \sigma^{*}}-\rho_{\sigma, \sigma^{*}} e_{\sigma, \sigma^{*}}}{\delta t}+\frac{1}{\left|D_{\sigma, \sigma^{*}}\right|} \sum_{\mathfrak{s} \in \partial D_{\sigma, \sigma^{*} \backslash \partial \Omega}}|\mathfrak{s}| \mathcal{E}_{D_{\sigma, \sigma^{*}, \mathfrak{s}}}+\frac{|\sigma|}{\left|D_{\sigma, \sigma^{*}}\right|} \mathcal{E}_{\sigma} \\
&+p_{\sigma, \sigma^{*}}\left(\boldsymbol{\nabla}_{\boldsymbol{d}} \cdot \overline{\mathbf{u}}\right)_{\sigma, \sigma^{*}}=\mathcal{R}_{\sigma, \sigma^{*}}, \quad \forall D_{\sigma, \sigma^{*}} \in \mathfrak{D}_{\text {ext }}
\end{aligned}
$$

where the flux $\mathcal{E}_{D_{\sigma, \sigma^{*}, \mathfrak{s}}}$ is defined by $(25), \boldsymbol{\nabla}_{\boldsymbol{d}} \cdot \overline{\mathbf{u}}$ is given by Definition 3.11 , and $\mathcal{R}_{\sigma, \sigma^{*}}$ by $(26)$. 


\subsection{Stability analysis}

We now turn to the stability analysis of the scheme. We exhibit a CFL-condition of the following form for interior diamonds (see (38) and (39))

$$
\frac{\delta t}{h_{D_{\sigma, \sigma^{*}}}}\left(\sum_{\mathfrak{s} \in \partial D_{\sigma, \sigma^{*}}}|\mathfrak{s}|\left[u_{D_{\sigma, \sigma^{*}, \mathfrak{s}}}\right]^{+}+\left(|\sigma|+\left|\sigma^{*}\right|\right) c\left(e_{\sigma, \sigma^{*}}\right)\right) \leqslant \mathcal{C}, \quad \forall D_{\sigma, \sigma^{*}} \in \mathfrak{D}_{\text {int }},
$$

(with $\mathcal{C} \in(0,1)$ depending only on $\operatorname{reg}(\mathfrak{T})$ and $\gamma$ ) which ensures that the numerical density and the internal energy remain non-negative. It also guarantees that the remainder terms $\mathcal{R}_{K}$ and $\mathcal{R}_{K^{*}}$, and thus $\mathcal{R}_{\sigma, \sigma^{*}}$, are kept positive. To this end, we shall use Lemma 3.2 and specifically property (7) of the numerical flux.

We do not claim, though, that the obtained condition is optimal. The value of the constant $\mathcal{C}$ (made explicit with (38) and (39)) is not used in practice to choose the time step. This result is however reassuring since it proves that the condition linking the time step and the mesh size involves the chararacteristic speeds in a classical way. Note it also involves the geometry of the cells, through the regularity parameter $\operatorname{reg}(\mathfrak{T})$. As reg $(\mathfrak{T})$ increases, which means dealing with a less regular mesh, containing elongated cells, the constant $\mathcal{C}$ tends to 0.

Proposition 3.13. Let $\rho_{\sigma, \sigma^{*}} \geqslant 0$. We assume that the following CFL-like conditions are satisfied

$$
\begin{aligned}
& \frac{\delta t}{\left|D_{\sigma, \sigma^{*}}\right|} \sum_{\mathfrak{s} \in \partial D_{\sigma, \sigma^{*}}}|\mathfrak{s}|\left[\lambda_{+}\left(c\left(e_{\mathfrak{s}}\right), u_{\left.D_{\sigma, \sigma^{*}, \mathfrak{s}}\right)}\right]^{+} \leqslant 1, \quad \forall D_{\sigma, \sigma^{*}} \in \mathfrak{D}_{\text {int }}\right.
\end{aligned}
$$

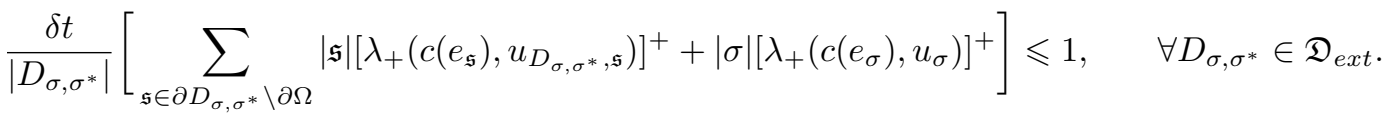

Then, the non negativity of the density $\rho_{\sigma, \sigma^{*}}$ is preserved: $\bar{\rho}_{\sigma, \sigma^{*}} \geqslant 0$.

Proof. Let $D_{\sigma, \sigma^{*}} \in \mathfrak{D}_{\text {int }}$. We go back to the mass conservation equation (9) and we make use of Lemma 3.2 and we are thus led to

$$
\begin{aligned}
\bar{\rho}_{\sigma, \sigma^{*}} & =\rho_{\sigma, \sigma^{*}}-\frac{\delta t}{\left|D_{\sigma, \sigma^{*}}\right|} \sum_{\mathfrak{s} \in \partial D_{\sigma, \sigma^{*}}}|\mathfrak{s}|\left(\mathcal{F}^{+}\left(\rho_{\sigma, \sigma^{*}}, u_{D_{\sigma, \sigma^{*}, \mathfrak{s}}}\right)+\mathcal{F}^{-}\left(\rho_{\sigma^{\prime}, \sigma^{*}}, u_{D_{\sigma, \sigma^{*}, \mathfrak{s}}}\right)\right) \\
& \geqslant \rho_{\sigma, \sigma^{*}}-\frac{\delta t}{\left|D_{\sigma, \sigma^{*}}\right|} \sum_{\mathfrak{s} \in \partial D_{\sigma, \sigma^{*}}}|\mathfrak{s}| \mathcal{F}^{+}\left(\rho_{\sigma, \sigma^{*}}, u_{\left.D_{\sigma, \sigma^{*}, \mathfrak{s}}\right)}\right. \\
& \geqslant \rho_{\sigma, \sigma^{*}}-\frac{\delta t}{\left|D_{\sigma, \sigma^{*}}\right|} \sum_{\mathfrak{s} \in \partial D_{\sigma, \sigma^{*}}}|\mathfrak{s}| \rho_{\sigma, \sigma^{*}}\left[\lambda _ { + } \left(c\left(e_{\mathfrak{s}}\right), u_{\left.\left.D_{\sigma, \sigma^{*}, \mathfrak{s}}\right)\right]^{+}} .\right.\right.
\end{aligned}
$$

With $\rho_{\sigma, \sigma^{*}} \geqslant 0$, the right hand side of this inequality remains non negative under the CFL-like condition (28). The proof for $D_{\sigma, \sigma^{*}} \in \mathfrak{D}_{\text {ext }}$ follows exactly the same lines and is left to the reader.

Remark 3.14. In order to compare the stability condition with the CFL condition obtained in $1 \mathrm{~d}$ and on MAC grids, see [6, 25], we remind the reader that $u_{D_{\sigma, \sigma^{*}, \mathfrak{s}}}=-u_{D_{\sigma^{\prime}, \sigma^{*}, \mathfrak{s}}}$ so that the characteristic speeds of the system satisfy:

$$
\begin{aligned}
{\left[\lambda _ { + } \left(c\left(e_{\mathfrak{s}}\right), u_{\left.\left.D_{\sigma, \sigma^{*}, \mathfrak{s}}\right)\right]^{+}}\right.\right.} & =\left[\lambda_{+}\left(c\left(e_{\mathfrak{s}}\right),-u_{\left.D_{\sigma^{\prime}, \sigma^{*}, \mathfrak{s}}\right)}\right]^{+}\right. \\
& =\left[-\lambda_{-}\left(c\left(e_{\mathfrak{s}}\right), u_{\left.D_{\sigma^{\prime}, \sigma^{*}, \mathfrak{s}}\right)}\right]^{+}=\left[\lambda_{-}\left(c\left(e_{\mathfrak{s}}\right), u_{\left.D_{\sigma^{\prime}, \sigma^{*}, \mathfrak{s}}\right)}\right]^{-} .\right.\right.
\end{aligned}
$$


It allows us to rewrite the conditions (28) in a form similar to what has been obtained on Cartesian grids. For instance, the first one can be recast as

$$
\frac{\delta t}{\left|D_{\sigma, \sigma^{*}}\right|}\left(\sum _ { \substack { \mathfrak { s } \in \partial D _ { \sigma , \sigma ^ { * } } \\ \mathfrak { s } \subset K } } | \mathfrak { s } | \left[\lambda_{+}\left(c\left(e_{\mathfrak{s}}\right), u_{\left.\left.D_{\sigma, \sigma^{*}, \mathfrak{s}}\right)\right]^{+}+} \sum_{\substack{\mathfrak{s} \in \partial D_{\sigma, \sigma^{*}} \\ \mathfrak{s} \subset L}}|\mathfrak{s}|\left[\lambda_{-}\left(c\left(e_{\mathfrak{s}}\right), u_{D_{\sigma^{\prime}, \sigma^{*}}, \mathfrak{s}}\right)\right]^{-}\right) \leqslant 1 .\right.\right.
$$

We now turn to the remainder term $\mathcal{R}_{K}$. The fact that $\mathcal{R}_{K}$ remains non-negative depends on the mesh-regularity coefficient reg $(\mathfrak{T})$, and the obtained condition is stronger than (28).

Proposition 3.15. Let us assume that the following CFL-like conditions are satisfied

$$
\begin{gathered}
\frac{\delta t}{\left|D_{\sigma, \sigma^{*}}\right|} \sum_{\mathfrak{s} \in \partial D_{\sigma, \sigma^{*}}}|\mathfrak{s}|\left[\lambda _ { + } \left(c\left(e_{\mathfrak{s}}\right), u_{\left.\left.D_{\sigma, \sigma^{*}, \mathfrak{s}}\right)\right]^{+}} \leqslant \frac{1}{1+\operatorname{reg}(\mathfrak{T})}, \quad \forall D_{\sigma, \sigma^{*}} \in \mathfrak{D}_{i n t},\right.\right. \\
\frac{\delta t}{\left|D_{\sigma, \sigma^{*}}\right|}\left(\sum _ { \mathfrak { s } \in \partial D _ { \sigma , \sigma ^ { * } } \backslash \partial \Omega } | \mathfrak { s } | \left[\lambda _ { + } \left(c\left(e_{\mathfrak{s}}\right), u_{\left.\left.D_{\sigma, \sigma^{*}, \mathfrak{s}}\right)\right]^{+}}\right.\right.\right. \\
\left.+|\sigma|\left[\lambda_{+}\left(c\left(e_{\sigma}\right), u_{\sigma}\right)\right]^{+}+|\sigma| \Lambda_{\sigma, \operatorname{Dir}}^{-}\right) \leqslant \frac{1}{\operatorname{reg}(\mathfrak{T})}, \quad \forall D_{\sigma, \sigma^{*}} \in \mathfrak{D}_{\text {ext }},
\end{gathered}
$$

with $\Lambda_{\sigma, \text { Dir }}^{-}=\frac{1}{2} \frac{\rho_{\text {Dir }}}{\rho_{\sigma, \sigma^{*}}}\left[\lambda_{-}\left(c\left(e_{\text {Dir }}\right), u_{\text {Dir }}\right)\right]^{-}$if $\sigma$ is a Dirichlet boudary and 0 otherwise. Then,

$$
\mathcal{R}_{K} \geqslant 0 \forall K \in \mathfrak{M}, \quad \mathcal{R}_{K^{*}} \geqslant 0 \forall K^{*} \in \mathfrak{M}^{*} \cup \partial \mathfrak{M}^{*} \quad \text { and } \quad \mathcal{R}_{\sigma, \sigma^{*}} \geqslant 0 \forall D_{\sigma, \sigma^{*}} \in \mathfrak{D} .
$$

Proof. Let us split the proof into two parts depending on the type of cell we consider.

a) Let $K \in \mathfrak{M}$. By using the averaged mass equation in Proposition 3.5, the remainder term $\mathcal{R}_{K}$ in Definition 3.9 can be rewritten as

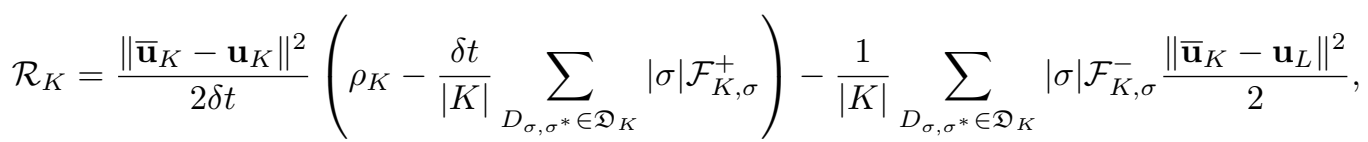

where the last contribution is non negative since $\mathcal{F}_{K, \sigma}^{-} \leqslant 0$. Hence, we get

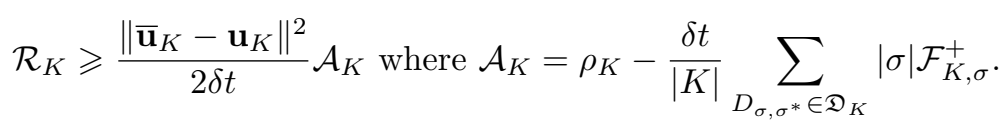

Having $\mathcal{A}_{K} \geqslant 0$ is thus enough to ensure $\mathcal{R}_{K} \geqslant 0$. Going back to Definition 3.4 for $\rho_{K}$ and to (15) for the expression of $\mathcal{F}_{K, \sigma}^{+}$, we modify the expression of $\mathcal{A}_{K}$, and we arrive at

$$
\begin{aligned}
\mathcal{A}_{K}= & \sum_{D_{\sigma, \sigma^{*}} \in \mathfrak{D}_{K} \cap \mathfrak{D}_{\text {ext }}}\left(\frac{\left|D_{\sigma, \sigma^{*}} \cap K\right|}{|K|} \rho_{\sigma, \sigma^{*}}-\frac{\delta t}{|K|}|\sigma| \mathcal{F}_{\sigma}^{+}\right) \\
+ & \sum_{D_{\sigma, \sigma^{*}} \in \mathfrak{D}_{K} \cap \mathfrak{D}_{i n t}}\left(\frac{\left|D_{\sigma, \sigma^{*}} \cap K\right|}{|K|} \rho_{\sigma, \sigma^{*}}-\frac{\delta t}{|K|} \frac{\left|D_{\sigma, \sigma^{*}} \cap K\right|}{\left|D_{\sigma, \sigma^{*}}\right|} \sum_{\substack{\mathfrak{s} \in \partial D_{\sigma, \sigma^{*}} \\
\mathfrak{s} \subset L}}|\mathfrak{s}| \mathcal{F}_{D_{\sigma, \sigma^{*}, \mathfrak{s}}^{+}}\right. \\
& \left.+\frac{\delta t}{|K|} \frac{\left|D_{\sigma, \sigma^{*}} \cap L\right|}{\left|D_{\sigma, \sigma^{*}}\right|} \sum_{\substack{\mathfrak{s} \in \partial D_{\sigma, \sigma^{*}} \\
\mathfrak{s} \subset K}}|\mathfrak{s}| \mathcal{F}_{D_{\sigma, \sigma^{*}, \mathfrak{s}}^{-}}\right) .
\end{aligned}
$$


Adding and substracting $\frac{\left|D_{\sigma, \sigma^{*}} \cap K\right|}{\left|D_{\sigma, \sigma^{*}}\right|} \sum_{\substack{\mathfrak{s} \in \partial D_{\sigma, \sigma^{*}} \\ \mathfrak{s} \subset K}}|\mathfrak{s}| \mathcal{F}_{D_{\sigma, \sigma^{*}, \mathfrak{s}}}^{+}$leads to:

$$
\begin{aligned}
\mathcal{A}_{K}= & \sum_{D_{\sigma, \sigma^{*}} \in \mathfrak{D}_{K} \cap \mathcal{D}_{\text {ext }}}\left(\frac{\left|D_{\sigma, \sigma^{*}} \cap K\right|}{|K|} \rho_{\sigma, \sigma^{*}}-\frac{\delta t}{|K|}|\sigma| \mathcal{F}_{\sigma}^{+}\right) \\
& +\sum_{D_{\sigma, \sigma^{*}} \in \mathfrak{D}_{K} \cap \mathfrak{D}_{i n t}} \frac{\left|D_{\sigma, \sigma^{*}} \cap K\right|}{|K|}\left(\rho_{\sigma, \sigma^{*}}-\frac{\delta t}{\left|D_{\sigma, \sigma^{*}}\right|} \sum_{\mathfrak{s} \in \partial D_{\sigma, \sigma^{*}}}|\mathfrak{s}| \mathcal{F}_{D_{\sigma, \sigma^{*}, \mathfrak{s}}^{+}}\right) \\
& +\frac{\delta t}{|K|} \sum_{D_{\sigma, \sigma^{*}} \in \mathfrak{D}_{K} \cap \mathfrak{D}_{i n t}} \frac{1}{\left|D_{\sigma, \sigma^{*}}\right|} \sum_{\substack{\mathfrak{s} \in \partial D_{\sigma, \sigma^{*}} \\
\mathfrak{s} \subset K}}|\mathfrak{s}|\left(\left|D_{\sigma, \sigma^{*}} \cap K\right| \mathcal{F}_{D_{\sigma, \sigma^{*}, \mathfrak{s}}^{+}}+\left|D_{\sigma, \sigma^{*}} \cap L\right| \mathcal{F}_{D_{\sigma, \sigma^{*}, \mathfrak{s}}}^{-}\right) .
\end{aligned}
$$

Let us start by rewriting the third sum. By using the equality $\left|D_{\sigma, \sigma^{*}} \cap L\right|=\left|D_{\sigma, \sigma^{*}}\right|-\left|D_{\sigma, \sigma^{*}} \cap K\right|$, we have

$$
\begin{aligned}
\sum_{D_{\sigma, \sigma^{*}} \in \mathfrak{D}_{K} \cap \mathfrak{D}_{i n t}} \frac{1}{\left|D_{\sigma, \sigma^{*}}\right|} \sum_{\substack{\mathfrak{s} \in \partial D_{\sigma, \sigma^{*}} \\
\mathfrak{s} \subset K}}|\mathfrak{s}|\left(\left|D_{\sigma, \sigma^{*}} \cap K\right| \mathcal{F}_{D_{\sigma, \sigma^{*}, \mathfrak{s}}^{+}}^{+}+\left|D_{\sigma, \sigma^{*}} \cap L\right| \mathcal{F}_{D_{\sigma, \sigma^{*}, \mathfrak{s}}}\right) \\
=\sum_{D_{\sigma, \sigma^{*}} \in \mathfrak{D}_{K} \cap \mathfrak{D}_{i n t}} \frac{1}{\left|D_{\sigma, \sigma^{*}}\right|} \sum_{\substack{\mathfrak{s} \in \partial D_{\sigma, \sigma^{*}} \\
\mathfrak{s} \subset K}}|\mathfrak{s}|\left(\left|D_{\sigma, \sigma^{*}} \cap K\right| \mathcal{F}_{D_{\sigma, \sigma^{*}, \mathfrak{s}}}^{|\cdot|}+\left|D_{\sigma, \sigma^{*}}\right| \mathcal{F}_{D_{\sigma, \sigma^{*}, \mathfrak{s}}}\right) .
\end{aligned}
$$

Since the flux $\mathcal{F}_{D_{\sigma, \sigma^{*}, \mathfrak{s}}}$ is conservative, we get

$$
\sum_{D_{\sigma, \sigma^{*}} \in \mathfrak{D}_{K} \cap \mathfrak{D}_{i n t}} \sum_{\mathfrak{s} \in \partial D_{\sigma, \sigma^{*}}}|\mathfrak{s}| \mathcal{F}_{D_{\sigma, \sigma^{*}, \mathfrak{s}}}=-\sum_{D_{\sigma, \sigma^{*}} \in \mathfrak{D}_{K} \cap \mathfrak{D}_{\text {ext }}} \sum_{\mathfrak{s} \in \partial D_{\sigma, \sigma *} \backslash \partial \Omega}|\mathfrak{s}| \mathcal{F}_{D_{\sigma, \sigma^{*}, \mathfrak{s}},}
$$

and thus

$$
\begin{aligned}
\sum_{D_{\sigma, \sigma^{*}} \in \mathfrak{D}_{K} \cap \mathfrak{D}_{i n t}} \sum_{\substack{\mathfrak{s} \in \partial D_{\sigma, \sigma^{*}} \\
\mathfrak{s} \subset K}}|\mathfrak{s}| \mathcal{F}_{D_{\sigma, \sigma^{*}, \mathfrak{s}}}^{-}= & -\sum_{D_{\sigma, \sigma^{*}} \in \mathfrak{D}_{K} \cap \mathfrak{D}_{i n t} \mathfrak{s} \in \partial D_{\sigma, \sigma^{*}}}|\mathfrak{s}| \mathcal{F}_{D_{\sigma, \sigma^{*}, \mathfrak{s}}^{+}} \sum_{D_{\sigma, \sigma^{*}} \in \mathfrak{D}_{K} \cap \mathfrak{D}_{e x t} \mathfrak{s} \in \partial D_{\sigma, \sigma^{*}} \backslash \partial \Omega}|\mathfrak{s}| \mathcal{F}_{D_{\sigma, \sigma^{*}, \mathfrak{s}}} .
\end{aligned}
$$

This equality can be used to rewrite the last term in (32). Finally, plugging this result in the definition of $\mathcal{A}_{K}$ yields

$$
\begin{aligned}
\mathcal{A}_{K}= & \sum_{D_{\sigma, \sigma^{*}} \in \mathfrak{D}_{K} \cap \mathcal{D}_{\text {ext }}}\left(\frac{\left|D_{\sigma, \sigma^{*}} \cap K\right|}{|K|} \rho_{\sigma, \sigma^{*}}-\frac{\delta t}{|K|}|\sigma| \mathcal{F}_{\sigma}^{+}-\frac{\delta t}{\mid D_{\sigma, \sigma^{*} \mid}} \sum_{\mathfrak{s} \in \partial D_{\sigma, \sigma^{*}} \backslash \partial \Omega}|\mathfrak{s}| \mathcal{F}_{D_{\sigma, \sigma^{*}, \mathfrak{s}}}\right) \\
& +\sum_{D_{\sigma, \sigma^{*}} \in \mathfrak{D}_{K} \cap \mathfrak{D}_{i n t}} \frac{\left|D_{\sigma, \sigma^{*}} \cap K\right|}{|K|}\left(\rho_{\sigma, \sigma^{*}}-\frac{\delta t}{\left|D_{\sigma, \sigma^{*}}\right|} \sum_{\mathfrak{s} \in \partial D_{\sigma, \sigma^{*}}}|\mathfrak{s}| \mathcal{F}_{D_{\sigma, \sigma^{*}, \mathfrak{s}}^{+}}\right) \\
& +\frac{\delta t}{|K|}\left(\sum_{D_{\sigma, \sigma^{*}} \in \mathfrak{D}_{K} \cap \mathfrak{D}_{\text {int }}} \frac{\left|D_{\sigma, \sigma^{*}} \cap K\right|}{\left|D_{\sigma, \sigma^{*}}\right|} \sum_{\substack{\mathfrak{s} \in \partial D_{\sigma, \sigma^{*}} \\
\mathfrak{s} \subset K}}|\mathfrak{s}| \mathcal{F}_{D_{\sigma, \sigma^{*}, \mathfrak{s}}}^{|\cdot|} \sum_{D_{\sigma, \sigma^{*}} \in \mathfrak{D}_{K} \cap \mathfrak{D}_{\text {int }}} \sum_{\substack{\mathfrak{s} \in \partial D_{\sigma, \sigma^{*}} \\
\mathfrak{s} \subset K}}|\mathfrak{s}| \mathcal{F}_{D_{\sigma, \sigma^{*}, \mathfrak{s}}}^{+}\right) .
\end{aligned}
$$

We are now going to establish a bound from below for $A_{K}$. To this end, we proceed as follows. Since $\mathcal{F}^{|\cdot|} \geqslant 0$ and $\mathcal{F} \leqslant \mathcal{F}^{+}$, we get

$$
\mathcal{A}_{K} \geqslant \mathcal{B}_{K}^{\text {ext }}+\mathcal{B}_{K}^{\text {int }},
$$


with

$$
\mathcal{B}_{K}^{e x t}=\sum_{D_{\sigma, \sigma^{*}} \in \mathfrak{D}_{K} \cap \mathfrak{D}_{e x t}}\left(\frac{\left|D_{\sigma, \sigma^{*}} \cap K\right|}{|K|} \rho_{\sigma, \sigma^{*}}-\frac{\delta t}{|K|}|\sigma| \mathcal{F}_{\sigma}^{+}-\frac{\delta t}{\left|D_{\sigma, \sigma^{*}}\right|} \sum_{\mathfrak{s} \in \partial D_{\sigma, \sigma^{*}} \backslash \partial \Omega}|\mathfrak{s}| \mathcal{F}_{D_{\sigma, \sigma^{*}, \mathfrak{s}}^{+}}\right),
$$

and

$$
\begin{aligned}
\mathcal{B}_{K}^{\text {int }}=\sum_{D_{\sigma, \sigma^{*}} \in \mathfrak{D}_{K} \cap \mathfrak{D}_{i n t}} & \frac{\left|D_{\sigma, \sigma^{*}} \cap K\right|}{|K|}\left(\rho_{\sigma, \sigma^{*}}-\frac{\delta t}{\left|D_{\sigma, \sigma^{*}}\right|} \sum_{\mathfrak{s} \in \partial D_{\sigma, \sigma^{*}}}|\mathfrak{s}| \mathcal{F}_{D_{\sigma, \sigma^{*}, \mathfrak{s}}}^{+}\right) \\
& -\frac{\delta t}{|K|} \sum_{D_{\sigma, \sigma^{*}} \in \mathfrak{D}_{K} \cap \mathfrak{D}_{\text {int }}} \sum_{\substack{\mathfrak{s} \in \partial D_{\sigma, \sigma^{*}} \\
\mathfrak{s} \subset K}}|\mathfrak{s}| \mathcal{F}_{D_{\sigma, \sigma^{*}, \mathfrak{s}}}^{+}
\end{aligned}
$$

Substracting the non negative term $\sum_{D_{\sigma, \sigma^{*}} \in \mathfrak{D}_{K} \cap \mathfrak{D}_{\text {int }} \mathfrak{s} \in \partial D_{\mathfrak{s} \subset L}}|\mathfrak{s}| \mathcal{F}_{D_{\sigma, \sigma^{*}, \mathfrak{s}}}^{+}$to $\mathcal{B}_{K}^{\text {int }}$, we get

$$
\begin{aligned}
\mathcal{B}_{K}^{\text {int }} \geqslant & \sum_{D_{\sigma, \sigma^{*}} \in \mathfrak{D}_{K} \cap \mathfrak{D}_{i n t}} \frac{\left|D_{\sigma, \sigma^{*}} \cap K\right|}{|K|}\left(\rho_{\sigma, \sigma^{*}}-\frac{\delta t}{\mid D_{\sigma, \sigma^{*} \mid}} \sum_{\mathfrak{s} \in \partial D_{\sigma, \sigma^{*}}}|\mathfrak{s}| \mathcal{F}_{D_{\sigma, \sigma^{*}, \mathfrak{s}}^{+}}\right) \\
-\frac{\delta t}{|K|} \sum_{D_{\sigma, \sigma^{*}} \in \mathfrak{D}_{K} \cap \mathfrak{D}_{i n t}} \sum_{\mathfrak{s} \in \partial D_{\sigma, \sigma^{*}}}|\mathfrak{s}| \mathcal{F}_{D_{\sigma, \sigma^{*}, \mathfrak{s}}^{+}} & \\
& \geqslant \sum_{D_{\sigma, \sigma^{*}} \in \mathfrak{D}_{K} \cap \mathfrak{D}_{i n t}} \frac{\left|D_{\sigma, \sigma^{*}} \cap K\right|}{|K|} \rho_{\sigma, \sigma^{*}}\left(1-\frac{\delta t}{\left|D_{\sigma, \sigma^{*}}\right|}\left(1+\frac{\left|D_{\sigma, \sigma^{*}}\right|}{\left|D_{\sigma, \sigma^{*}} \cap K\right|}\right) \sum_{\mathfrak{s} \in \partial D_{\sigma, \sigma^{*}}}|\mathfrak{s}| \frac{\mathcal{F}_{D_{\sigma, \sigma^{*}, \mathfrak{s}}}^{+}}{\rho_{\sigma, \sigma^{*}}}\right) .
\end{aligned}
$$

By using the definition of mass flux (8) and Lemma 3.2 we end up with

$$
\mathcal{B}_{K}^{i n t} \geqslant \sum_{D_{\sigma, \sigma^{*}} \in \mathfrak{D}_{K} \cap \mathcal{D}_{i n t}|K|} \frac{\left|D_{\sigma, \sigma^{*}} \cap K\right|}{|K|} \rho_{\sigma, \sigma^{*}}\left(1-\frac{\delta t}{\left|D_{\sigma, \sigma^{*}}\right|}\left(1+\frac{\left|D_{\sigma, \sigma^{*}}\right|}{\left|D_{\sigma, \sigma^{*}} \cap K\right|}\right) \sum_{\mathfrak{s} \in \partial D_{\sigma, \sigma^{*}}}|\mathfrak{s}|\left[\lambda_{+}\left(c\left(e_{\mathfrak{s}}\right), u_{\left.\left.D_{\sigma, \sigma^{*}, \mathfrak{s}}\right)\right]^{+}}\right) .\right.\right.
$$

Therefore $\mathcal{B}_{K}^{i n t} \geqslant 0$ holds when

$$
1 \geqslant \frac{\delta t}{\left|D_{\sigma, \sigma^{*}}\right|}\left(1+\frac{\left|D_{\sigma, \sigma^{*}}\right|}{\left|D_{\sigma, \sigma^{*}} \cap K\right|}\right) \sum_{\mathfrak{s} \in \partial D_{\sigma, \sigma^{*}}}|\mathfrak{s}|\left[\lambda_{+}\left(c\left(e_{\mathfrak{s}}\right), u_{D_{\sigma, \sigma^{*}, \mathfrak{s}}}\right)\right]^{+} .
$$

This inequality holds when (29) is fulfilled since $\operatorname{reg}(\mathfrak{T}) \geqslant \frac{\left|D_{\sigma, \sigma^{*}}\right|}{\left|D_{\sigma, \sigma^{*}} \cap K\right|}$.

We now turn to the study of $\mathcal{B}_{K}^{\text {ext }}$. Since, for all $D_{\sigma, \sigma^{*}} \in \mathfrak{D}_{K} \cap \mathfrak{D}_{\text {ext }},\left|D_{\sigma, \sigma^{*}} \cap K\right|=\left|D_{\sigma, \sigma^{*}}\right|$, we have

$$
\begin{aligned}
\mathcal{B}_{K}^{\text {ext }}= & \sum_{D_{\sigma, \sigma^{*}} \in \mathfrak{D}_{K} \cap \mathfrak{D}_{\text {ext }}} \frac{\left|D_{\sigma, \sigma^{*}}\right|}{|K|} \rho_{\sigma, \sigma^{*}}\left(1-\frac{\delta t}{\left|D_{\sigma, \sigma^{*}}\right|}|\sigma| \frac{\mathcal{F}_{\sigma}^{+}}{\rho_{\sigma, \sigma^{*}}}-\frac{\delta t}{\left|D_{\sigma, \sigma^{*}}\right|} \frac{|K|}{\left|D_{\sigma, \sigma^{*}}\right|} \sum_{\mathfrak{s} \in \partial D_{\sigma, \sigma^{*}} \backslash \partial \Omega}|\mathfrak{s}| \frac{\mathcal{F}_{D_{\sigma, \sigma^{*}, \mathfrak{s}}}^{+}}{\rho_{\sigma, \sigma^{*}}}\right) \\
\geqslant & \sum_{D_{\sigma, \sigma^{*}} \in \mathfrak{D}_{K} \cap \mathfrak{D}_{\text {ext }}} \frac{\left|D_{\sigma, \sigma^{*}}\right|}{|K|} \rho_{\sigma, \sigma^{*}}\left(1-\frac{\delta t}{\left|D_{\sigma, \sigma^{*}}\right|}|\sigma|\left[\lambda_{+}\left(c\left(e_{\sigma}\right), u_{\sigma}\right)\right]^{+}\right. \\
& \quad-\frac{\delta t}{\left|D_{\sigma, \sigma^{*}}\right|} \operatorname{reg}(\mathfrak{T}) \sum_{\mathfrak{s} \in \partial D_{\sigma, \sigma^{*} \backslash \partial \Omega}}|\mathfrak{s}|\left[\lambda_{+}\left(c\left(e_{\mathfrak{s}}\right), u_{\left.\left.D_{\sigma, \sigma^{*}, \mathfrak{s}}\right)\right]^{+}}\right) .\right.
\end{aligned}
$$


Therefore $\mathcal{B}_{K}^{\text {ext }} \geqslant 0$ holds when

$$
1 \geqslant \frac{\delta t}{\left|D_{\sigma, \sigma^{*}}\right|}\left(|\sigma|\left[\lambda_{+}\left(c\left(e_{\sigma}\right), u_{\sigma}\right)\right]^{+}+\operatorname{reg}(\mathfrak{T}) \sum_{\mathfrak{s} \in \partial D_{\sigma, \sigma^{*}} \backslash \partial \Omega}|\mathfrak{s}|\left[\lambda_{+}\left(c\left(e_{\mathfrak{s}}\right), u_{\left.\left.D_{\sigma, \sigma^{*}, \mathfrak{s}}\right)\right]^{+}}\right)\right.\right.
$$

This inequality holds when (30) is fulfilled since reg $(\mathfrak{T}) \geqslant 1$. Thus, $\mathcal{R}_{K} \geqslant 0$, for any $K \in \mathfrak{M}$.

b) We now turn to the study of $\mathcal{R}_{K^{*}}$ for $K^{*} \in \mathfrak{M}^{*} \cup \partial \mathfrak{M}^{*}$. The proof follows the same lines. By using the averaged mass equation in Proposition 3.5, the remainder term $\mathcal{R}_{K^{*}}$ in Definition 3.9 can be rewritten as

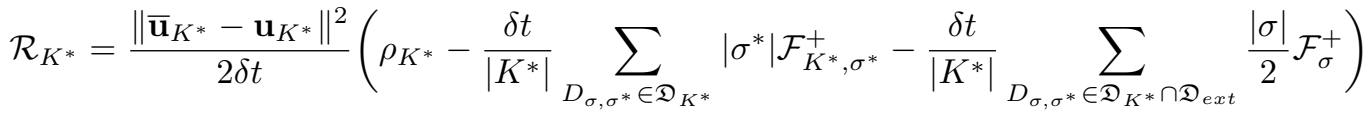

$$
\begin{aligned}
& -\frac{1}{\left|K^{*}\right|} \sum_{D_{\sigma, \sigma^{*}} \in \mathfrak{D}_{K^{*}}}\left|\sigma^{*}\right| \mathcal{F}_{K^{*}, \sigma^{*}}^{-} \frac{\left\|\overline{\mathbf{u}}_{K^{*}}-\mathbf{u}_{L^{*}}\right\|^{2}}{2} \\
& -\frac{1}{\left|K^{*}\right|} \sum_{D_{\sigma, \sigma^{*}} \in \mathfrak{D}_{K^{*}} \cap \mathfrak{D}_{\text {ext }}} \frac{|\sigma|}{2} \mathcal{F}_{\sigma}^{-} \frac{\left\|\overline{\mathbf{u}}_{K^{*}}-\mathbf{u}_{\sigma}\right\|^{2}}{2}
\end{aligned}
$$

where the two last contribution are non negative since $\mathcal{F}_{K^{*}, \sigma^{*}}^{-} \leqslant 0$ and $\mathcal{F}_{\sigma}^{-} \leqslant 0$. Hence, we get

$$
\mathcal{R}_{K^{*}} \geqslant \frac{\left\|\overline{\mathbf{u}}_{K^{*}}-\mathbf{u}_{K^{*}}\right\|^{2}}{2 \delta t} \mathcal{A}_{K^{*}}
$$

where

$$
\mathcal{A}_{K^{*}}=\rho_{K^{*}}-\frac{\delta t}{\left|K^{*}\right|} \sum_{D_{\sigma, \sigma^{*}} \in \mathfrak{D}_{K^{*}}}\left|\sigma^{*}\right| \mathcal{F}_{K^{*}, \sigma^{*}}^{+}-\frac{\delta t}{\left|K^{*}\right|} \sum_{D_{\sigma, \sigma^{*}} \in \mathfrak{D}_{K^{*}} \cap \mathfrak{D}_{e x t}} \frac{|\sigma|}{2} \mathcal{F}_{\sigma}^{+} .
$$

Having $\mathcal{A}_{K^{*}} \geqslant 0$ is thus enough to ensure $\mathcal{R}_{K^{*}} \geqslant 0$. Going back to Definition 3.4 for $\rho_{K^{*}}$ and to (16) and (18) for $\mathcal{F}_{K^{*}, \sigma^{*}}^{+}$, we modify the expression of $\mathcal{A}_{K^{*}}$, and we arrive at

$$
\begin{aligned}
& \mathcal{A}_{K^{*}}=\sum_{D_{\sigma, \sigma^{*}} \in \mathfrak{D}_{K^{*}} \cap \mathfrak{D}_{e x t}}\left(\frac{\left|D_{\sigma, \sigma^{*}} \cap K^{*}\right|}{\left|K^{*}\right|} \rho_{\sigma, \sigma^{*}}-\frac{\delta t}{\left|K^{*}\right|} \frac{|\sigma|}{2} \mathcal{F}_{\sigma}^{+}\right. \\
& -\frac{\delta t}{\left|K^{*}\right|} \frac{\left|D_{\sigma, \sigma^{*}} \cap K^{*}\right|}{\left|D_{\sigma, \sigma^{*}}\right|}\left|\mathfrak{s}_{K L^{*}}\right| \mathcal{F}_{D_{\sigma, \sigma^{*}, \mathfrak{s}_{K L}}^{+}}+\frac{\delta t}{\left|K^{*}\right|} \frac{\left|D_{\sigma, \sigma^{*}} \cap L^{*}\right|}{\left|D_{\sigma, \sigma^{*}}\right|}\left|\mathfrak{s}_{K K^{*}}\right| \mathcal{F}_{D_{\sigma, \sigma^{*}, \mathfrak{s}_{K} K^{*}}}^{-} \\
& \left.-\frac{\delta t}{\left|K^{*}\right|} \frac{\left|D_{\sigma, \sigma^{*}} \cap K^{*}\right|}{2\left|D_{\sigma, \sigma^{*}}\right|}|\sigma| \mathcal{F}_{\sigma}^{+}+\frac{\delta t}{\left|K^{*}\right|} \frac{\left|D_{\sigma, \sigma^{*}} \cap L^{*}\right|}{2\left|D_{\sigma, \sigma^{*}}\right|}|\sigma| \mathcal{F}_{\sigma}^{-}\right) \\
& +\sum_{D_{\sigma, \sigma^{*}} \in \mathfrak{D}_{K^{*}} \cap \mathfrak{D}_{i n t}}\left(\frac{\left|D_{\sigma, \sigma^{*}} \cap K^{*}\right|}{\left|K^{*}\right|} \rho_{\sigma, \sigma^{*}}-\frac{\delta t}{\left|K^{*}\right|} \frac{\left|D_{\sigma, \sigma^{*}} \cap K^{*}\right|}{\left|D_{\sigma, \sigma^{*}}\right|} \sum_{\substack{\mathfrak{s} \in \partial D_{\sigma, \sigma^{*}} \\
\mathfrak{s} \subset L^{*}}}|\mathfrak{s}| \mathcal{F}_{D_{\sigma, \sigma^{*}, \mathfrak{s}}^{+}}^{+}\right. \\
& \left.+\frac{\delta t}{\left|K^{*}\right|} \frac{\left|D_{\sigma, \sigma^{*}} \cap L^{*}\right|}{\left|D_{\sigma, \sigma^{*}}\right|} \sum_{\substack{\mathfrak{s} \in \partial D_{\sigma, \sigma^{*}} \\
\mathfrak{s} \subset K^{*}}}|\mathfrak{s}| \mathcal{F}_{D_{\sigma, \sigma^{*}, \mathfrak{s}}}^{-}\right)
\end{aligned}
$$


Adding and substracting $\frac{\left|D_{\sigma, \sigma^{*}} \cap K^{*}\right|}{\left|D_{\sigma, \sigma^{*}}\right|} \sum_{\substack{\mathfrak{s} \in \partial D_{\sigma, \sigma^{*}} \\ \mathfrak{s} \subset K^{*}}}|\mathfrak{s}| \mathcal{F}_{D_{\sigma, \sigma^{*}, \mathfrak{s}}^{+}}^{+}$leads to:

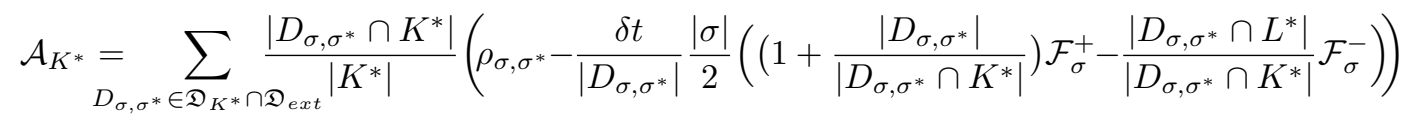

$$
\begin{aligned}
& +\frac{\delta t}{\left|K^{*}\right|} \sum_{D_{\sigma, \sigma^{*}} \in \mathfrak{D}_{K^{*}} \cap \mathcal{D}_{e x t}}\left(-\frac{\left|D_{\sigma, \sigma^{*}} \cap K^{*}\right|}{\left|D_{\sigma, \sigma^{*}}\right|}\left|\mathfrak{s}_{K L^{*}}\right| \mathcal{F}_{D_{\sigma, \sigma^{*}, \mathfrak{s}_{K L^{*}}}^{+}}+\frac{\left|D_{\sigma, \sigma^{*}} \cap L^{*}\right|}{\left|D_{\sigma, \sigma^{*}}\right|}\left|\mathfrak{s}_{K K^{*}}\right| \mathcal{F}_{D_{\sigma, \sigma^{*}, \mathfrak{s}_{K} K^{*}}^{-}}\right) \\
& +\sum_{D_{\sigma, \sigma^{*}} \in \mathfrak{D}_{K^{*}} \cap \mathfrak{D}_{i n t}} \frac{\left|D_{\sigma, \sigma^{*}} \cap K^{*}\right|}{\left|K^{*}\right|}\left(\rho_{\sigma, \sigma^{*}}-\frac{\delta t}{\left|D_{\sigma, \sigma^{*}}\right|} \sum_{\mathfrak{s} \in \partial D_{\sigma, \sigma^{*}}}|\mathfrak{s}| \mathcal{F}_{D_{\sigma, \sigma^{*}, \mathfrak{s}}^{+}}\right) \\
& +\frac{\delta t}{\left|K^{*}\right|} \sum_{D_{\sigma, \sigma^{*}} \in \mathfrak{D}_{K^{*}} \cap \mathfrak{D}_{i n t}} \frac{1}{\left|D_{\sigma, \sigma^{*}}\right|} \sum_{\substack{\mathfrak{s} \in \partial D_{\sigma, \sigma^{*}} \\
\mathfrak{s} \subset K^{*}}}|\mathfrak{s}|\left(\left|D_{\sigma, \sigma^{*}} \cap K^{*}\right| \mathcal{F}_{D_{\sigma, \sigma^{*}, \mathfrak{s}}}^{+}+\left|D_{\sigma, \sigma^{*}} \cap L^{*}\right| \mathcal{F}_{D_{\sigma, \sigma^{*}, \mathfrak{s}}}^{-}\right) .
\end{aligned}
$$

Let us start by rewriting the fourth sum. Using equality $\left|D_{\sigma, \sigma^{*}} \cap L^{*}\right|=\left|D_{\sigma, \sigma^{*}}\right|-\left|D_{\sigma, \sigma^{*}} \cap K^{*}\right|$, we have

$$
\begin{aligned}
\sum_{D_{\sigma, \sigma^{*}} \in \mathfrak{D}_{K^{*}} \cap \mathfrak{D}_{i n t}} \frac{1}{\left|D_{\sigma, \sigma^{*}}\right|} \sum_{\substack{\mathfrak{s} \in \partial D_{\sigma, \sigma^{*}} \\
\mathfrak{s} \subset K^{*}}}|\mathfrak{s}|\left(\left|D_{\sigma, \sigma^{*}} \cap K^{*}\right| \mathcal{F}_{D_{\sigma, \sigma^{*}, \mathfrak{s}}}^{+}+\left|D_{\sigma, \sigma^{*}} \cap L^{*}\right| \mathcal{F}_{D_{\sigma, \sigma^{*}, \mathfrak{s}}}\right) \\
=\sum_{D_{\sigma, \sigma^{*}} \in \mathfrak{D}_{K^{*} \cap \mathfrak{D}_{i n t}}} \frac{1}{\left|D_{\sigma, \sigma^{*}}\right|} \sum_{\substack{\mathfrak{s} \in \partial D_{\sigma, \sigma^{*}} \\
\mathfrak{s} \subset K^{*}}}|\mathfrak{s}|\left(\left|D_{\sigma, \sigma^{*}} \cap K^{*}\right| \mathcal{F}_{D_{\sigma, \sigma^{*}, \mathfrak{s}}}^{|\cdot|}+\left|D_{\sigma, \sigma^{*}}\right| \mathcal{F}_{D_{\sigma, \sigma^{*}, \mathfrak{s}}}^{-}\right) .
\end{aligned}
$$

Since the flux $\mathcal{F}_{D_{\sigma, \sigma^{*}, \mathfrak{s}}}$ is conservative, we get

$$
\sum_{D_{\sigma, \sigma^{*}} \in \mathfrak{D}_{K^{*}} \cap \mathcal{D}_{i n t} \sum_{\mathfrak{s} \in \partial D_{\sigma, \sigma^{*}}}|\mathfrak{s}| \mathcal{F}_{D_{\sigma, \sigma^{*}, \mathfrak{s}}}=-\sum_{D_{\sigma, \sigma^{*}} \in \mathfrak{D}_{K^{*}} \cap \mathfrak{D}_{e x t}} \sum_{\mathfrak{s} \in \partial D_{\sigma, \sigma^{*}} \backslash \partial \Omega} \mid \mathfrak{s} \subset K^{*}} \mid \mathcal{F}_{D_{\sigma, \sigma^{*}, \mathfrak{s}}},
$$

and thus

$$
\begin{aligned}
& \sum_{D_{\sigma, \sigma^{*}} \in \mathfrak{D}_{K^{*}} \cap \mathfrak{D}_{i n t}} \sum_{\substack{\mathfrak{s} \in \partial D_{\sigma, \sigma^{*}} \\
\mathfrak{s} \subset K^{*}}}|\mathfrak{s}| \mathcal{F}_{D_{\sigma, \sigma^{*}, \mathfrak{s}}}^{-} \\
& =-\sum_{D_{\sigma, \sigma^{*}} \in \mathfrak{D}_{K^{*}} \cap \mathfrak{D}_{i n t} \mathfrak{s} \in \partial D_{\sigma, \sigma^{*}}}|\mathfrak{s}| \mathcal{F}_{D_{\sigma, \sigma^{*}, \mathfrak{s}}}^{+}-\sum_{D_{\sigma, \sigma^{*}} \in \mathfrak{D}_{K^{*}} \cap \mathfrak{D}_{e x t} \mathfrak{s} \in \partial D_{\sigma, \sigma^{*}} \backslash \partial \Omega}|\mathfrak{s}| \mathcal{F}_{D_{\sigma, \sigma^{*}, \mathfrak{s}}} .
\end{aligned}
$$


Plugging this result in the definition of $\mathcal{A}_{K^{*}}$ yields

$$
\begin{aligned}
& \mathcal{A}_{K^{*}}=\sum_{D_{\sigma, \sigma^{*}} \in \mathfrak{D}_{K^{*}} \cap \mathfrak{D}_{e x t}} \frac{\left|D_{\sigma, \sigma^{*}} \cap K^{*}\right|}{\left|K^{*}\right|}\left(\rho_{\sigma, \sigma^{*}}-\frac{\delta t}{\left|D_{\sigma, \sigma^{*}}\right|} \frac{|\sigma|}{2}\left(\left(1+\frac{\left|D_{\sigma, \sigma^{*}}\right|}{\left|D_{\sigma, \sigma^{*}} \cap K^{*}\right|}\right) \mathcal{F}_{\sigma}^{+}-\frac{\left|D_{\sigma, \sigma^{*}} \cap L^{*}\right|}{\left|D_{\sigma, \sigma^{*}} \cap K^{*}\right|} \mathcal{F}_{\sigma}^{-}\right)\right) \\
& -\frac{\delta t}{\left|K^{*}\right|} \sum_{D_{\sigma, \sigma^{*}} \in \mathfrak{D}_{K^{*}} \cap \mathcal{D}_{e x t}}\left(\frac{D_{\sigma, \sigma^{*}} \cap K^{*} \mid}{\left|D_{\sigma, \sigma^{*}}\right|}\left|\mathfrak{s}_{K L^{*}}\right| \mathcal{F}_{D_{\sigma, \sigma^{*}, \mathfrak{s}_{K} L^{*}}^{+}}^{+}+\frac{\left|D_{\sigma, \sigma^{*}} \cap K^{*}\right|}{\left|D_{\sigma, \sigma^{*}}\right|}\left|\mathfrak{s}_{K K^{*}}\right| \mathcal{F}_{D_{\sigma, \sigma^{*}, \mathfrak{s}_{K} K^{*}}}^{-}+\left|\mathfrak{s}_{K K^{*}}\right| \mathcal{F}_{D_{\sigma, \sigma^{*}, \mathfrak{s}_{K K^{*}}}^{+}}\right) \\
& +\sum_{D_{\sigma, \sigma^{*}} \in \mathfrak{D}_{K^{*}} \cap \mathfrak{D}_{i n t}} \frac{\left|D_{\sigma, \sigma^{*}} \cap K^{*}\right|}{\left|K^{*}\right|}\left(\rho_{\sigma, \sigma^{*}}-\frac{\delta t}{\left|D_{\sigma, \sigma^{*}}\right|} \sum_{\mathfrak{s} \in \partial D_{\sigma, \sigma^{*}}}|\mathfrak{s}| \mathcal{F}_{D_{\sigma, \sigma^{*}, \mathfrak{s}}^{+}}\right) \\
& +\frac{\delta t}{\left|K^{*}\right|} \sum_{D_{\sigma, \sigma^{*}} \in \mathfrak{D}_{K^{*}} \cap \mathfrak{D}_{i n t}} \frac{1}{\left|D_{\sigma, \sigma^{*}}\right|} \sum_{\substack{\mathfrak{s} \in \partial D_{\sigma, \sigma^{*}} \\
\mathfrak{s} \subset K^{*}}}|\mathfrak{s}|\left|D_{\sigma, \sigma^{*}} \cap K^{*}\right| \mathcal{F}_{D_{\sigma, \sigma^{*}, \mathfrak{s}}}^{|\cdot|}
\end{aligned}
$$

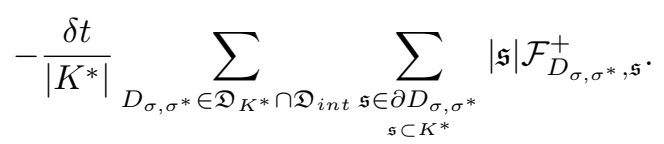

Since $\mathcal{F}^{|\cdot|} \geqslant 0$ and $\mathcal{F}_{D_{\sigma, \sigma^{*}, \mathfrak{s}_{K} K^{*}}}^{-} \leqslant 0$, we have

$$
\mathcal{A}_{K^{*}} \geqslant \mathcal{B}_{K^{*}}^{e x t}+\mathcal{B}_{K^{*}}^{\text {int }}
$$

with

$$
\begin{array}{r}
\mathcal{B}_{K^{*}}^{e x t}=\sum_{D_{\sigma, \sigma^{*}} \in \mathfrak{D}_{K^{*}} \cap \mathfrak{D}_{e x t}} \frac{\left|D_{\sigma, \sigma^{*}} \cap K^{*}\right|}{K^{*} \mid}\left(\rho_{\sigma, \sigma^{*}}-\frac{\delta t}{\left|D_{\sigma, \sigma^{*}}\right|} \frac{|\sigma|}{2}\left(\left(1+\frac{\left|D_{\sigma, \sigma^{*}}\right|}{\left|D_{\sigma, \sigma^{*}} \cap K^{*}\right|}\right) \mathcal{F}_{\sigma}^{+}-\frac{\left|D_{\sigma, \sigma^{*}} \cap L^{*}\right|}{\left|D_{\sigma, \sigma^{*}} \cap K^{*}\right|} \mathcal{F}_{\sigma}^{-}\right)\right) \\
-\frac{\delta t}{\left|K^{*}\right|} \sum_{D_{\sigma, \sigma^{*}} \in \mathfrak{D}_{K^{*}} \cap \mathfrak{D}_{e x t}}\left(\frac{\left|D_{\sigma, \sigma^{*}} \cap K^{*}\right|}{\left|D_{\sigma, \sigma^{*}}\right|}\left|\mathfrak{s}_{K L^{*}}\right| \mathcal{F}_{D_{\sigma, \sigma^{*}, \mathfrak{s}_{K L}}^{+}}^{+}+\left|\mathfrak{s}_{K K^{*}}\right| \mathcal{F}_{D_{\sigma, \sigma^{*}, \mathfrak{s}_{K} K^{*}}^{+}}\right)
\end{array}
$$

and

$$
\begin{aligned}
& \mathcal{B}_{K^{*}}^{i n t}=\sum_{D_{\sigma, \sigma^{*}} \in \mathfrak{D}_{K^{*}} \cap \mathfrak{D}_{i n t}} \frac{\left|D_{\sigma, \sigma^{*}} \cap K^{*}\right|}{\left|K^{*}\right|}\left(\rho_{\sigma, \sigma^{*}}-\frac{\delta t}{\left|D_{\sigma, \sigma^{*}}\right|} \sum_{\mathfrak{s} \in \partial D_{\sigma, \sigma^{*}}}|\mathfrak{s}| \mathcal{F}_{D_{\sigma, \sigma^{*}, \mathfrak{s}}^{+}}\right) \\
& -\frac{\delta t}{\left|K^{*}\right|} \sum_{D_{\sigma, \sigma^{*}} \in \mathfrak{D}_{K^{*}} \cap \mathfrak{D}_{i n t}} \sum_{\mathfrak{s} \in \partial D_{\sigma, \sigma^{*}}}|\mathfrak{s}| \mathcal{F}_{D_{\sigma, \sigma^{*}, \mathfrak{s}}^{+}}^{+} .
\end{aligned}
$$

Substracting the non negative term $\frac{\delta t}{\left|K^{*}\right|} \sum_{D_{\sigma, \sigma^{*}} \in \mathfrak{D}_{K^{*}} \cap \mathfrak{D}_{\text {int }} \mathfrak{s} \in \partial D_{\sigma, \sigma^{*}}}|\mathfrak{s}| \mathcal{F}_{D_{\sigma, \sigma^{*}, \mathfrak{s}}^{+}}^{+}$to $\mathcal{B}_{K^{*}}^{\text {int }}$, we get

$$
\begin{aligned}
\mathcal{B}_{K^{*}}^{\text {int }} \geqslant \sum_{D_{\sigma, \sigma^{*}} \in \mathfrak{D}_{K^{*}} \cap \mathfrak{D}_{i n t}} \frac{\left|D_{\sigma, \sigma^{*}} \cap K^{*}\right|}{\left|K^{*}\right|}\left(\rho_{\sigma, \sigma^{*}}\right. & \left.-\frac{\delta t}{\left|D_{\sigma, \sigma^{*}}\right|} \sum_{\mathfrak{s} \in \partial D_{\sigma, \sigma^{*}}}|\mathfrak{s}| \mathcal{F}_{D_{\sigma, \sigma^{*}, \mathfrak{s}}^{+}}\right) \\
& -\frac{\delta t}{\left|K^{*}\right|} \sum_{D_{\sigma, \sigma^{*}} \in \mathfrak{D}_{K^{*}} \cap \mathfrak{D}_{i n t} \in \mathfrak{s} \in \partial D_{\sigma, \sigma^{*}}}|\mathfrak{s}| \mathcal{F}_{D_{\sigma, \sigma^{*}, \mathfrak{s}}^{+}} \\
\geqslant & \sum_{D_{\sigma, \sigma^{*}} \in \mathfrak{D}_{K^{*}} \cap \mathfrak{D}_{i n t}} \frac{\left|D_{\sigma, \sigma^{*}} \cap K^{*}\right|}{\left|K^{*}\right|} \rho_{\sigma, \sigma^{*}}\left(1-\frac{\delta t}{\left|D_{\sigma, \sigma^{*}}\right|}\left(1+\frac{\left|D_{\sigma, \sigma^{*}}\right|}{\left|D_{\sigma, \sigma^{*}} \cap K^{*}\right|}\right) \sum_{\mathfrak{s} \in \partial D_{\sigma, \sigma^{*}}}|\mathfrak{s}| \frac{\mathcal{F}_{D_{\sigma, \sigma^{*}, \mathfrak{s}}}^{+}}{\rho_{\sigma, \sigma^{*}}}\right) .
\end{aligned}
$$

By using Lemma 3.2 we end up with

$$
\mathcal{B}_{K^{*}}^{i n t} \geqslant \sum_{D_{\sigma, \sigma^{*}} \in \mathfrak{D}_{K^{*}} \cap \mathfrak{D}_{i n t}\left|K^{*}\right|} \frac{\left|D_{\sigma, \sigma^{*}} \cap K^{*}\right|}{\rho_{\sigma, \sigma^{*}}}\left(1-\frac{\delta t}{\left|D_{\sigma, \sigma^{*}}\right|}\left(1+\frac{\left|D_{\sigma, \sigma^{*}}\right|}{\left|D_{\sigma, \sigma^{*}} \cap K^{*}\right|}\right) \sum_{\mathfrak{s} \in \partial D_{\sigma, \sigma^{*}}}|\mathfrak{s}|\left[\lambda_{+}\left(c\left(e_{\mathfrak{s}}\right), u_{\left.D_{\sigma, \sigma^{*}, \mathfrak{s}}\right)}\right]^{+}\right) .\right.
$$


Therefore $\mathcal{B}_{K^{*}}^{\text {int }} \geqslant 0$ holds when

$$
1 \geqslant \frac{\delta t}{\left|D_{\sigma, \sigma^{*}}\right|}\left(1+\frac{\left|D_{\sigma, \sigma^{*}}\right|}{\left|D_{\sigma, \sigma^{*}} \cap K^{*}\right|}\right) \sum_{\mathfrak{s} \in \partial D_{\sigma, \sigma^{*}}}|\mathfrak{s}|\left[\lambda _ { + } \left(c\left(e_{\mathfrak{s}}\right), u_{\left.\left.D_{\sigma, \sigma^{*}, \mathfrak{s}}\right)\right]^{+}} .\right.\right.
$$

This inequality holds when (29) is fulfilled since reg $(\mathfrak{T}) \geqslant \frac{\left|D_{\sigma, \sigma^{*}}\right|}{\left|D_{\sigma, \sigma^{*}} \cap K^{*}\right|}$.

We now turn to the study of $\mathcal{B}_{K^{*}}^{\text {ext }}$. We have

$$
\begin{aligned}
& \mathcal{B}_{K^{*}}^{e x t}=\sum_{D_{\sigma, \sigma^{*}} \in \mathfrak{D}_{K^{*}} \cap \mathcal{D}_{e x t}} \frac{\left|D_{\sigma, \sigma^{*}} \cap K^{*}\right|}{\left|K^{*}\right|}\left(\rho_{\sigma, \sigma^{*}}-\frac{\delta t}{\left|D_{\sigma, \sigma^{*}}\right|} \frac{|\sigma|}{2}\left(\left(1+\frac{\left|D_{\sigma, \sigma^{*}}\right|}{\left|D_{\sigma, \sigma^{*}} \cap K^{*}\right|}\right) \mathcal{F}_{\sigma}^{+}-\frac{\left|D_{\sigma, \sigma^{*}} \cap L^{*}\right|}{\left|D_{\sigma, \sigma^{*}} \cap K^{*}\right|} \mathcal{F}_{\sigma}^{-}\right)\right. \\
& \left.-\frac{\delta t}{\left|D_{\sigma, \sigma^{*}}\right|}\left(\left|\mathfrak{s}_{K L^{*}}\right| \mathcal{F}_{D_{\sigma, \sigma^{*}, \mathfrak{s}_{K L^{*}}}^{+}}+\frac{\left|D_{\sigma, \sigma^{*}}\right|}{\left|D_{\sigma, \sigma^{*}} \cap K^{*}\right|}\left|\mathfrak{s}_{K K^{*}}\right| \mathcal{F}_{D_{\sigma, \sigma^{*}, \mathfrak{s}_{K K}}^{+}}^{+}\right)\right) \\
& \geqslant \sum_{D_{\sigma, \sigma^{*}} \in \mathfrak{D}_{K^{*}} \cap \mathfrak{D}_{e x t}\left|K^{*}\right|} \frac{\left|D_{\sigma, \sigma^{*}} \cap K^{*}\right|}{\mid r, \sigma^{*}}\left(1-\frac{\delta t}{\left|D_{\sigma, \sigma^{*}}\right|} \frac{|\sigma|}{2}\left((1+\operatorname{reg}(\mathfrak{T})) \frac{\mathcal{F}_{\sigma}^{+}}{\rho_{\sigma, \sigma^{*}}}+(\operatorname{reg}(\mathfrak{T})-1) \frac{-\mathcal{F}_{\sigma}^{-}}{\rho_{\sigma, \sigma^{*}}}\right)\right. \\
& \left.-\frac{\delta t}{\left|D_{\sigma, \sigma^{*}}\right|} \operatorname{reg}(\mathfrak{T})\left(\left|\mathfrak{s}_{K L^{*}}\right| \frac{\mathcal{F}_{D_{\sigma, \sigma^{*}, \mathfrak{s}_{K L}}^{+}}^{+}}{\rho_{\sigma, \sigma^{*}}}+\left|\mathfrak{s}_{K K^{*}}\right| \frac{\mathcal{F}_{D_{\sigma, \sigma^{*}, \mathfrak{s}_{K K}}}^{+}}{\rho_{\sigma, \sigma^{*}}}\right)\right)
\end{aligned}
$$

Therefore, since reg $(\mathfrak{T}) \geqslant 2, \mathcal{B}_{K}^{\text {ext }} \geqslant 0$ holds when (30) is fulfilled. Thus, $\mathcal{R}_{K^{*}} \geqslant 0$.

That $\mathcal{R}_{D_{\sigma, \sigma^{*}}}$ is non negative follows from the fact that $\mathcal{R}_{K}, \mathcal{R}_{L}, \mathcal{R}_{K^{*}}$ and $\mathcal{R}_{L^{*}}$ are all non negative.

We are now able to exhibit the CFL-like condition that ensures the non-negativity of the internal energy $e_{\sigma, \sigma^{*}}$.

Proposition 3.16. Let $e_{\sigma, \sigma^{*}} \geqslant 0$ and $\rho_{\sigma, \sigma^{*}} \geqslant 0, \forall D_{\sigma, \sigma^{*}} \in \mathfrak{D}$. Let assume that the following CFL-like conditions are satisfied

$$
\begin{aligned}
& \frac{\delta t}{\left|D_{\sigma, \sigma^{*}}\right|}\left(\operatorname{reg}(\mathfrak{T})^{2} \frac{\left|\sigma^{*}\right|+|\sigma|}{\sqrt{2}} c\left(e_{\sigma, \sigma^{*}}\right)+\sum_{\mathfrak{s} \in \partial D_{\sigma, \sigma^{*}}}|\mathfrak{s}|\left(\left[u_{D_{\sigma, \sigma^{*}, \mathfrak{s}}}\right]^{+}+c\left(e_{\mathfrak{s}}\right)\right)\right) \\
& \quad \leqslant \min \left(\frac{1}{\gamma}, \frac{1}{1+\operatorname{reg}(\mathfrak{T})}\right), \quad \forall D_{\sigma, \sigma^{*}} \in \mathfrak{D}_{\text {int }} \\
& \frac{\delta t}{\left|D_{\sigma, \sigma^{*}}\right|}\left(\operatorname{reg}(\mathfrak{T})^{2} \frac{\left|\sigma^{*}\right|+|\sigma|}{\sqrt{2}} c\left(e_{\sigma, \sigma^{*}}\right)+\sum_{\mathfrak{s} \in \partial D_{\sigma, \sigma^{*} \backslash \partial \Omega}}|\mathfrak{s}|\left(\left[u_{\left.\left.D_{\sigma, \sigma^{*}, \mathfrak{s}}\right]^{+}+c\left(e_{\mathfrak{s}}\right)\right)}\right.\right.\right. \\
& \left.\quad+|\sigma|\left[\lambda_{+}\left(c\left(e_{\sigma}\right), u_{\sigma}\right)\right]^{+}+|\sigma| \Lambda_{\sigma, \text { Dir }}^{-}\right) \leqslant \min \left(\frac{1}{\gamma}, \frac{1}{1+\operatorname{reg}(\mathfrak{T})}\right), \quad \forall D_{\sigma, \sigma^{*}} \in \mathfrak{D}_{\text {ext }}
\end{aligned}
$$

with $\Lambda_{\sigma, \text { Dir }}^{-}=\frac{1}{2} \frac{\rho_{\text {Dir }}}{\rho_{\sigma, \sigma^{*}}}\left[\lambda_{-}\left(c\left(e_{\text {Dir }}\right), u_{\text {Dir }}\right)\right]^{-}$if $\sigma$ is a Dirichlet boudary and 0 otherwise. Then, the non negativity of the internal energy is preserved: we have $\bar{e}_{\sigma, \sigma^{*}} \geqslant 0, \forall D_{\sigma, \sigma^{*}} \in \mathfrak{D}$.

Proof. We start by observing that (38), (39) implies (28) and (29), (30). In particular, thanks to Proposition 3.13, we have $\bar{\rho}_{\sigma, \sigma^{*}} \geqslant 0, \forall D_{\sigma, \sigma^{*}} \in \mathfrak{D}$. Next, we turn to the non negativity of $\bar{e}_{\sigma, \sigma^{*}}$; we follow the arguments in [25].

Let us write $\left(\boldsymbol{\nabla}_{\boldsymbol{d}} \cdot \overline{\mathbf{u}}\right)_{\sigma, \sigma^{*}}$ as in Lemma 3.12 and then apply the Young inequality for each four terms. For $X \in \mathfrak{M}$ and $i \in\{0,1\}$ we write

$$
\begin{array}{r}
(-1)^{i} p_{\sigma, \sigma^{*}} \overline{\mathbf{u}}_{X} \cdot \mathbf{n}_{K, \sigma}=(-1)^{i}(\gamma-1)\left[\rho_{\sigma, \sigma^{*}} e_{\sigma, \sigma^{*}}\left(\overline{\mathbf{u}}_{X}-\mathbf{u}_{X}\right) \cdot \mathbf{n}_{K, \sigma}+\rho_{\sigma, \sigma^{*}} e_{\sigma, \sigma^{*}} \mathbf{u}_{X} \cdot \mathbf{n}_{K, \sigma}\right] \\
\geqslant-\rho_{\sigma, \sigma^{*}}\left[\frac{c\left(e_{\sigma, \sigma^{*}}\right)}{2 \sqrt{2} \gamma}\left\|\overline{\mathbf{u}}_{X}-\mathbf{u}_{X}\right\|^{2}+(\gamma-1) e_{\sigma, \sigma^{*}}\left(\frac{c\left(e_{\sigma, \sigma^{*}}\right)}{\sqrt{2}}-(-1)^{i} \mathbf{u}_{X} \cdot \mathbf{n}_{K, \sigma}\right)\right],
\end{array}
$$


so that

$$
\begin{aligned}
-\frac{\delta t|\sigma|}{2\left|D_{\sigma, \sigma^{*}}\right|} p_{\sigma, \sigma^{*}}\left(\overline{\mathbf{u}}_{L}-\overline{\mathbf{u}}_{K}\right) \cdot \mathbf{n}_{K, \sigma} & \geqslant-\frac{\delta t|\sigma|}{2 \mid D_{\sigma, \sigma^{*} \mid}} \rho_{\sigma, \sigma^{*}} \frac{c\left(e_{\sigma, \sigma^{*}}\right)}{2 \sqrt{2} \gamma}\left(\left\|\overline{\mathbf{u}}_{K}-\mathbf{u}_{K}\right\|^{2}+\left\|\overline{\mathbf{u}}_{L}-\mathbf{u}_{L}\right\|^{2}\right) \\
& -\frac{\delta t|\sigma|}{2\left|D_{\sigma, \sigma^{*}}\right|}(\gamma-1) \rho_{\sigma, \sigma^{*}} e_{\sigma, \sigma^{*}}\left(\frac{2 c\left(e_{\sigma, \sigma^{*}}\right)}{\sqrt{2}}+\left(\mathbf{u}_{L}-\mathbf{u}_{K}\right) \cdot \mathbf{n}_{K, \sigma}\right) .
\end{aligned}
$$

Exactly the same computation for $K^{*}$ and $L^{*}$ leads to

$$
\begin{aligned}
-\frac{\delta t\left|\sigma^{*}\right|}{2\left|D_{\sigma, \sigma^{*}}\right|} p_{\sigma, \sigma^{*}}\left(\overline{\mathbf{u}}_{L^{*}}-\overline{\mathbf{u}}_{K^{*}}\right) \cdot \mathbf{n}_{K^{*}, \sigma^{*}} \geqslant & -\frac{\delta t\left|\sigma^{*}\right|}{2\left|D_{\sigma, \sigma^{*}}\right|} \rho_{\sigma, \sigma^{*}} \frac{c\left(e_{\sigma, \sigma^{*}}\right)}{2 \sqrt{2} \gamma}\left(\left\|\overline{\mathbf{u}}_{K^{*}}-\mathbf{u}_{K^{*}}\right\|^{2}+\left\|\overline{\mathbf{u}}_{L^{*}}-\mathbf{u}_{L^{*}}\right\|^{2}\right) \\
& -\frac{\delta t\left|\sigma^{*}\right|}{2\left|D_{\sigma, \sigma^{*}}\right|}(\gamma-1) \rho_{\sigma, \sigma^{*}} e_{\sigma, \sigma^{*}}\left(\frac{2 c\left(e_{\sigma, \sigma^{*}}\right)}{\sqrt{2}}+\left(\mathbf{u}_{L^{*}}-\mathbf{u}_{K^{*}}\right) \cdot \mathbf{n}_{K^{*}, \sigma^{*}}\right) .
\end{aligned}
$$
$\mathfrak{D}_{\text {ext }}$.

We now split the proof into two steps, depending on the localisation of $D_{\sigma, \sigma^{*}}$ in $\mathfrak{D}_{\text {int }}$ or

a) Suppose that $D_{\sigma, \sigma^{*}} \in \mathfrak{D}_{\text {int }}$ with $\sigma=K \mid L$ and $\sigma^{*}=K^{*} \mid L^{*}$. Owing to (27), we get

$$
\bar{\rho}_{\sigma, \sigma^{*}} \bar{e}_{\sigma, \sigma^{*}} \geqslant T_{0}+T_{K}+T_{L}+T_{K^{*}}+T_{L^{*}}
$$

with

$$
\begin{aligned}
T_{0}=\rho_{\sigma, \sigma^{*}} e_{\sigma, \sigma^{*}} & -\rho_{\sigma, \sigma^{*}} e_{\sigma, \sigma^{*}} \frac{\delta t|\sigma|}{2\left|D_{\sigma, \sigma^{*}}\right|}(\gamma-1)\left(\frac{2 c\left(e_{\sigma, \sigma^{*}}\right)}{\sqrt{2}}+\left(\mathbf{u}_{L}-\mathbf{u}_{K}\right) \cdot \mathbf{n}_{K, \sigma}\right) \\
& -\rho_{\sigma, \sigma^{*}} e_{\sigma, \sigma^{*}} \frac{\delta t\left|\sigma^{*}\right|}{2\left|D_{\sigma, \sigma^{*}}\right|}(\gamma-1)\left(\frac{2 c\left(e_{\sigma, \sigma^{*}}\right)}{\sqrt{2}}+\left(\mathbf{u}_{L^{*}}-\mathbf{u}_{K^{*}}\right) \cdot \mathbf{n}_{K^{*}, \sigma^{*}}\right) \\
& -\frac{\delta t}{\left|D_{\sigma, \sigma^{*} \mid}\right|} \sum_{\mathfrak{s} \in \partial D_{\sigma, \sigma^{*}}}|\mathfrak{s}| \mathcal{E}_{D_{\sigma, \sigma^{*}, \mathfrak{s}}},
\end{aligned}
$$

and

$$
\begin{gathered}
T_{X}=\frac{\delta t}{2\left|D_{\sigma, \sigma^{*}}\right|}\left(\left|D_{\sigma, \sigma^{*}} \cap X\right| \mathcal{R}_{X}-\frac{|\sigma|}{2 \sqrt{2} \gamma} \rho_{\sigma, \sigma^{*}} c\left(e_{\sigma, \sigma^{*}}\right)\left\|\overline{\mathbf{u}}_{X}-\mathbf{u}_{X}\right\|^{2}\right), \quad X \in\{K, L\}, \\
T_{X^{*}}=\frac{\delta t}{2\left|D_{\sigma, \sigma^{*}}\right|}\left(\left|D_{\sigma, \sigma^{*}} \cap X^{*}\right| \mathcal{R}_{X^{*}}-\frac{\left|\sigma^{*}\right|}{2 \sqrt{2} \gamma} \rho_{\sigma, \sigma^{*}} c\left(e_{\sigma, \sigma^{*}}\right)\left\|\overline{\mathbf{u}}_{X^{*}}-\mathbf{u}_{X^{*}}\right\|^{2}\right), \quad X^{*} \in\left\{K^{*}, L^{*}\right\} .
\end{gathered}
$$

In order to guaranty $\bar{e}_{\sigma, \sigma^{*}} \geqslant 0$ it is sufficient to ensure that these five terms are non negative. Using Lemma 3.12 on $\left(\boldsymbol{\nabla}_{\boldsymbol{d}} \cdot \mathbf{u}\right)_{\sigma, \sigma^{*}}, T_{0}$ becomes

$$
\begin{aligned}
T_{0}=\rho_{\sigma, \sigma^{*}} e_{\sigma, \sigma^{*}}\left(1-\frac{\delta t}{\left|D_{\sigma, \sigma^{*}}\right|}(\gamma-1)\left(\frac{\left|\sigma^{*}\right|+|\sigma|}{\sqrt{2}} c\left(e_{\sigma, \sigma^{*}}\right)\right.\right. & \left.\left.+\sum_{\mathfrak{s} \in \partial D_{\sigma, \sigma^{*}}}|\mathfrak{s}| u_{D_{\sigma, \sigma^{*}, \mathfrak{s}}}\right)\right) \\
& -\frac{\delta t}{\left|D_{\sigma, \sigma^{*}}\right|} \sum_{\mathfrak{s} \in \partial D_{\sigma, \sigma^{*}}}|\mathfrak{s}| \mathcal{E}_{D_{\sigma, \sigma^{*}, \mathfrak{s}}}
\end{aligned}
$$

Owing to Lemma 3.2 , we have

$$
\mathcal{E}_{D_{\sigma, \sigma^{*}, \mathfrak{s}}}=e_{\sigma, \sigma^{*}} \mathcal{F}_{D_{\sigma, \sigma^{*}, \mathfrak{s}}^{+}}^{+}+e_{\sigma^{\prime}, \sigma^{*}} \mathcal{F}_{D_{\sigma^{\prime}, \sigma^{*}, \mathfrak{s}}^{-}} \leqslant e_{\sigma, \sigma^{*}} \mathcal{F}_{D_{\sigma, \sigma^{*}, \mathfrak{s}}}^{+} \leqslant \rho_{\sigma, \sigma^{*}} e_{\sigma, \sigma^{*}}\left[\lambda_{+}\left(c\left(e_{\mathfrak{s}}\right), u_{\left.D_{\sigma, \sigma^{*}, \mathfrak{s}}\right)}\right]^{+}\right.
$$

and this allows us to bound $T_{0}$ from below as

$$
\begin{aligned}
T_{0} \geqslant \rho_{\sigma, \sigma^{*}} e_{\sigma, \sigma^{*}}\left(1-\frac{\delta t}{\left|D_{\sigma, \sigma^{*}}\right|}(\gamma-1)\left(\frac{\left|\sigma^{*}\right|+|\sigma|}{\sqrt{2}} c\left(e_{\sigma, \sigma^{*}}\right)+\sum_{\mathfrak{s} \in \partial D_{\sigma, \sigma^{*}}}|\mathfrak{s}| u_{D_{\sigma, \sigma^{*}, \mathfrak{s}}}\right)\right. \\
\left.-\frac{\delta t}{\left|D_{\sigma, \sigma^{*}}\right|} \sum_{\mathfrak{s} \in \partial D_{\sigma, \sigma^{*}}}|\mathfrak{s}|\left[\lambda_{+}\left(c\left(e_{\mathfrak{s}}\right), u_{D_{\sigma, \sigma^{*}, \mathfrak{s}}}\right)\right]^{+}\right) .
\end{aligned}
$$


Finally, since $u_{D_{\sigma, \sigma^{*}, \mathfrak{s}}} \leqslant\left[u_{D_{\sigma, \sigma^{*}, \mathfrak{s}}}\right]^{+}$and $\left[\lambda_{+}\left(c\left(e_{\mathfrak{s}}\right), u_{\left.D_{\sigma, \sigma^{*}, \mathfrak{s}}\right)}\right]^{+} \leqslant\left[u_{D_{\sigma, \sigma^{*}, \mathfrak{s}}}\right]^{+}+c\left(e_{\mathfrak{s}}\right)\right.$, the fact that $T_{0} \geqslant 0$ follows from

$$
\begin{aligned}
1 \geqslant \frac{\delta t}{\left|D_{\sigma, \sigma^{*}}\right|}(\gamma-1)\left(\frac{\left|\sigma^{*}\right|+|\sigma|}{\sqrt{2}} c\left(e_{\sigma, \sigma^{*}}\right)\right. & \left.+\sum_{\mathfrak{s} \in \partial D_{\sigma, \sigma^{*}}}|\mathfrak{s}|\left[u_{D_{\sigma, \sigma^{*}, \mathfrak{s}}}\right]^{+}\right) \\
& +\frac{\delta t}{\left|D_{\sigma, \sigma^{*}}\right|} \sum_{\mathfrak{s} \in \partial D_{\sigma, \sigma^{*}}}|\mathfrak{s}|\left(\left[u_{D_{\sigma, \sigma^{*}, \mathfrak{s}}}\right]^{+}+c\left(e_{\mathfrak{s}}\right)\right) .
\end{aligned}
$$

This inequality is ensured by the assumption (38). Next, we turn to the term $T_{K}$. We use the bound for $R_{K}$ given in the proof of Proposition 3.15. Using the same notation, owing to (31), we can write

$$
T_{K} \geqslant \frac{\left\|\overline{\mathbf{u}}_{K}-\mathbf{u}_{K}\right\|^{2}}{4\left|D_{\sigma, \sigma^{*}}\right|}\left(\left|D_{\sigma, \sigma^{*}} \cap K\right| \mathcal{A}_{K}-\rho_{\sigma, \sigma^{*}} c\left(e_{\sigma, \sigma^{*}}\right) \frac{\delta t|\sigma|}{\sqrt{2} \gamma}\right) .
$$

Moreover, we proved that $\mathcal{A}_{K} \geqslant \mathcal{B}_{K}^{\text {int }}+\mathcal{B}_{K}^{\text {ext }}$ with $\mathcal{B}_{K}^{\text {ext }} \geqslant 0$ since (30) holds. Thus, going back to (33), we have $\mathcal{A}_{K} \geqslant \mathcal{B}_{K}^{i n t} \geqslant \sum_{D_{\sigma^{\prime}, \sigma^{*}} \in \mathfrak{D}_{K} \cap \mathfrak{D}_{\text {int }}} \frac{\left|D_{\sigma^{\prime}, \sigma^{*^{\prime}}} \cap K\right|}{|K|} \rho_{\sigma^{\prime}, \sigma^{\prime}} P_{\sigma^{\prime}, \sigma^{*^{\prime}}}$ where

$$
P_{\sigma^{\prime}, \sigma^{*^{\prime}}}=1-\frac{\delta t}{\left|D_{\sigma^{\prime}, \sigma^{*^{\prime}}}\right|}\left(1+\frac{\left|D_{\sigma^{\prime}, \sigma^{*^{\prime}}}\right|}{\left|D_{\sigma^{\prime}, \sigma^{*^{\prime}}} \cap K\right|}\right) \sum_{\mathfrak{s} \in \partial D_{\sigma^{\prime}, \sigma^{*^{\prime}}}}|\mathfrak{s}|\left[\lambda _ { + } \left(c\left(e_{\mathfrak{s}}\right), u_{\left.\left.D_{\sigma^{\prime}, \sigma^{*^{\prime}}, \mathfrak{s}}\right)\right]^{+} .} .\right.\right.
$$

However, since (29) holds, we also proved that $P_{\sigma^{\prime}, \sigma^{* \prime}} \geqslant 0$ for each $D_{\sigma^{\prime}, \sigma^{*^{\prime}}} \in \mathfrak{D}_{\text {int }}$ so that $\mathcal{A}_{K} \geqslant \frac{\mid D_{\sigma, \sigma^{*} \cap K \mid}}{|K|} \rho_{\sigma, \sigma^{*}} P_{\sigma, \sigma^{*}}$ and we obtain

$$
T_{K} \geqslant \frac{\left|D_{\sigma, \sigma^{*}} \cap K\right|^{2}}{4\left|D_{\sigma, \sigma^{*}}\right||K|} \rho_{\sigma, \sigma^{*}}\left\|\overline{\mathbf{u}}_{K}-\mathbf{u}_{K}\right\|^{2} \tilde{P}_{\sigma, \sigma^{*}},
$$

where

$$
\tilde{P}_{\sigma, \sigma^{*}}=P_{\sigma, \sigma^{*}}-c\left(e_{\sigma, \sigma^{*}}\right) \frac{\delta t}{\sqrt{2} \gamma} \frac{|\sigma||K|}{\left|D_{\sigma, \sigma^{*}} \cap K\right|^{2}} .
$$

To ensure that $T_{K}$ is non negative, it is sufficient to prove that $\tilde{P}_{\sigma, \sigma^{*}} \geqslant 0$. We first observe that

$$
\frac{|K|\left|D_{\sigma, \sigma^{*}}\right|}{\left|D_{\sigma, \sigma^{*}} \cap K\right|^{2}}=\frac{|K|}{\left|D_{\sigma, \sigma^{*}}\right|} \times\left(\frac{\left|D_{\sigma, \sigma^{*}}\right|}{\left|D_{\sigma, \sigma^{*}} \cap K\right|}\right)^{2} \leqslant \operatorname{reg}(\mathfrak{T})^{3} .
$$

It leads to

$$
\begin{aligned}
& \tilde{P}_{\sigma, \sigma^{*}} \geqslant P_{\sigma, \sigma^{*}}-\frac{\delta t}{\left|D_{\sigma, \sigma^{*}}\right|} \frac{\operatorname{reg}(\mathfrak{T})^{3}}{\sqrt{2} \gamma}|\sigma| c\left(e_{\sigma, \sigma^{*}}\right)
\end{aligned}
$$

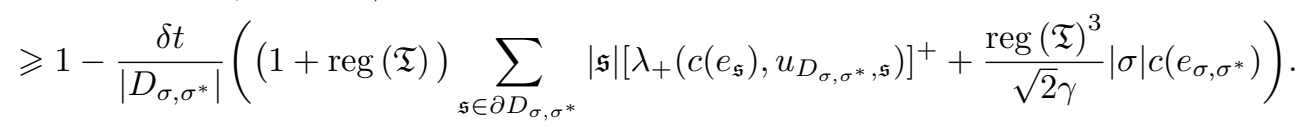

Thus, $T_{K}$ is non negative as soon as

$$
\frac{\delta t}{\left|D_{\sigma, \sigma^{*}}\right|}\left((1+\operatorname{reg}(\mathfrak{T})) \sum_{\mathfrak{s} \in \partial D_{\sigma, \sigma^{*}}}|\mathfrak{s}|\left[\lambda_{+}\left(c\left(e_{\mathfrak{s}}\right), u_{\left.D_{\sigma, \sigma^{*}, \mathfrak{s}}\right)}\right]^{+}+\frac{\operatorname{reg}(\mathfrak{T})^{3}}{\sqrt{2} \gamma}|\sigma| c\left(e_{\sigma, \sigma^{*}}\right)\right) \leqslant 1,\right.
$$

and this inequality holds thanks to assumption (38). Obviously, exactly the same result holds for $T_{L}$. Concerning $T_{K^{*}}$ (and $T_{L^{*}}$ ), owing to (36), we first observe that, as for $\mathcal{A}_{K}$, the following bound holds

$$
\mathcal{A}_{K^{*}} \geqslant \mathcal{B}_{K^{*}}^{i n t} \geqslant \frac{\left|D_{\sigma, \sigma^{*}} \cap K\right|}{|K|} \rho_{\sigma, \sigma^{*}} P_{\sigma, \sigma^{*}},
$$

(with the same definition of $P_{\sigma, \sigma^{*}}$, i.e. given by (40)). Therefore, following the same lines, we 
obtain that $T_{K^{*}}$ and $T_{L^{*}}$ are non negative provided

$$
\frac{\delta t}{\left|D_{\sigma, \sigma^{*}}\right|}\left((1+\operatorname{reg}(\mathfrak{T})) \sum_{\mathfrak{s} \in \partial D_{\sigma, \sigma^{*}}}|\mathfrak{s}|\left[\lambda_{+}\left(c\left(e_{\mathfrak{s}}\right), u_{\left.D_{\sigma, \sigma^{*}, \mathfrak{s}}\right)}\right]^{+}+\frac{\operatorname{reg}(\mathfrak{T})^{3}}{\sqrt{2} \gamma}\left|\sigma^{*}\right| c\left(e_{\sigma, \sigma^{*}}\right)\right) \leqslant 1 .\right.
$$

This inequality is implied by assumption (38).

b) Suppose now that $D_{\sigma, \sigma^{*}} \in \mathfrak{D}_{e x t}$, we get $\bar{\rho}_{\sigma, \sigma^{*}} \bar{e}_{\sigma, \sigma^{*}} \geqslant T_{0}+T_{K}+T_{L}+T_{K^{*}}+T_{L^{*}}$ with

$$
\begin{aligned}
& T_{0}=\rho_{\sigma, \sigma^{*}} e_{\sigma, \sigma^{*}}-\rho_{\sigma, \sigma^{*}} e_{\sigma, \sigma^{*}} \frac{\delta t|\sigma|}{2 \mid D_{\sigma, \sigma^{*} \mid}}(\gamma-1)\left(\frac{2 c\left(e_{\sigma, \sigma^{*}}\right)}{\sqrt{2}}+\left(\mathbf{u}_{L}-\mathbf{u}_{K}\right) \mathbf{n}_{K, \sigma}\right) \\
& -\rho_{\sigma, \sigma^{*}} e_{\sigma, \sigma^{*}} \frac{\delta t\left|\sigma^{*}\right|}{2\left|D_{\sigma, \sigma^{*}}\right|}(\gamma-1)\left(\frac{2 c\left(e_{\sigma, \sigma^{*}}\right)}{\sqrt{2}}+\left(\mathbf{u}_{L^{*}}-\mathbf{u}_{K^{*}}\right) \mathbf{n}_{K^{*}, \sigma^{*}}\right) \\
& -\frac{\delta t}{\left|D_{\sigma, \sigma^{*}}\right|} \sum_{\mathfrak{s} \in \partial D_{\sigma, \sigma^{*}} \backslash \partial \Omega}|\mathfrak{s}| \mathcal{E}_{D_{\sigma, \sigma^{*}, \mathfrak{s}}}-\frac{\delta t}{\left|D_{\sigma, \sigma^{*}}\right|}|\sigma| \mathcal{E}_{\sigma},
\end{aligned}
$$

and

$$
\begin{gathered}
T_{X}=\frac{\delta t}{2\left|D_{\sigma, \sigma^{*}}\right|}\left(\left|D_{\sigma, \sigma^{*}} \cap X\right| \mathcal{R}_{X}-\frac{|\sigma|}{2 \sqrt{2} \gamma} \rho_{\sigma, \sigma^{*}} c\left(e_{\sigma, \sigma^{*}}\right)\left\|\overline{\mathbf{u}}_{X}-\mathbf{u}_{X}\right\|^{2}\right), \quad X \in\{K, L\} \\
T_{X^{*}}=\frac{\delta t}{2\left|D_{\sigma, \sigma^{*}}\right|}\left(\left|D_{\sigma, \sigma^{*}} \cap X^{*}\right| \mathcal{R}_{X^{*}}-\frac{\left|\sigma^{*}\right|}{2 \sqrt{2} \gamma} \rho_{\sigma, \sigma^{*}} c\left(e_{\sigma, \sigma^{*}}\right)\left\|\overline{\mathbf{u}}_{X^{*}}-\mathbf{u}_{X^{*}}\right\|^{2}\right) . \quad X^{*} \in\left\{K^{*}, L^{*}\right\}
\end{gathered}
$$

As previously, in order to guaranty $\bar{e}_{\sigma, \sigma^{*}} \geqslant 0$ it is sufficient to ensure that these five terms are non negative. We first note that the expression of $T_{0}$ is the same than in the case a) (that is $\left.D_{\sigma, \sigma^{*}} \in \mathfrak{D}_{\text {int }}\right)$ up to the additional boundary term $|\sigma| \mathcal{E}_{\sigma}$. Thus, following the same lines and using the inequalities $\mathcal{E}_{\sigma} \leqslant e_{\sigma, \sigma^{*}} \mathcal{F}_{\sigma}^{+} \leqslant \rho_{\sigma, \sigma^{*}} e_{\sigma, \sigma^{*}}\left[\lambda_{+}\left(c\left(e_{\sigma}\right), u_{\sigma}\right)\right]^{+}$we arrive at the conclusion that $T_{0}$ is non negative as soon as

$$
\begin{aligned}
& 1 \geqslant \frac{\delta t}{\left|D_{\sigma, \sigma^{*}}\right|}(\gamma-1)\left(\frac{\left|\sigma^{*}\right|+|\sigma|}{\sqrt{2}} c\left(e_{\sigma, \sigma^{*}}\right)+\sum_{\mathfrak{s} \in \partial D_{\sigma, \sigma^{*}}}|\mathfrak{s}|\left[u_{\left.D_{\sigma, \sigma^{*}, \mathfrak{s}}\right]^{+}}\right)\right. \\
& +\frac{\delta t}{\left|D_{\sigma, \sigma^{*}}\right|} \sum_{\mathfrak{s} \in \partial D_{\sigma, \sigma^{*}} \backslash \partial \Omega}|\mathfrak{s}|\left(\left[u_{D_{\sigma, \sigma^{*}, \mathfrak{s}}}\right]^{+}+c\left(e_{\mathfrak{s}}\right)\right)+\frac{\delta t}{\left|D_{\sigma, \sigma^{*}}\right|}|\sigma|\left[\lambda_{+}\left(c\left(e_{\sigma}\right), u_{\sigma}\right)\right]^{+} .
\end{aligned}
$$

This is ensured by assumption (39).

Next, we turn to the term $T_{K}$. As in the case a), we use the notation of the proof of Proposition 3.15. We have

$$
T_{K} \geqslant \frac{\left\|\overline{\mathbf{u}}_{K}-\mathbf{u}_{K}\right\|^{2}}{4\left|D_{\sigma, \sigma^{*}}\right|}\left(\left|D_{\sigma, \sigma^{*}} \cap K\right| \mathcal{A}_{K}-\rho_{\sigma, \sigma^{*}} c\left(e_{\sigma, \sigma^{*}}\right) \frac{\delta t|\sigma|}{\sqrt{2} \gamma}\right) .
$$

Moreover, we proved that $\mathcal{A}_{K} \geqslant \mathcal{B}_{K}^{\text {int }}+\mathcal{B}_{K}^{\text {ext }}$ with $\mathcal{B}_{K}^{\text {int }} \geqslant 0$ since (29) holds. Thus, going back to (34), we have $\mathcal{A}_{K} \geqslant \mathcal{B}_{K}^{e x t} \geqslant \sum_{D_{\sigma^{\prime}, \sigma^{*}} \in \mathfrak{D}_{K} \cap \mathfrak{D}_{e x t}} \frac{\left|D_{\sigma^{\prime}, \sigma^{*^{\prime}}}\right|}{|K|} \rho_{\sigma^{\prime}, \sigma^{\prime}} Q_{\sigma^{\prime}, \sigma^{*^{\prime}}}$ where

$$
Q_{\sigma^{\prime}, \sigma^{*^{\prime}}}=1-\frac{\delta t}{\left|D_{\sigma, \sigma^{*}}\right|}|\sigma|\left[\lambda_{+}\left(c\left(e_{\sigma}\right), u_{\sigma}\right)\right]^{+}-\frac{\delta t}{\left|D_{\sigma, \sigma^{*}}\right|} \operatorname{reg}(\mathfrak{T}) \sum_{\mathfrak{s} \in \partial D_{\sigma, \sigma^{*}} \backslash \partial \Omega}|\mathfrak{s}|\left[\lambda _ { + } \left(c\left(e_{\mathfrak{s}}\right), u_{\left.\left.D_{\sigma, \sigma^{*}, \mathfrak{s}}\right)\right]^{+}}\right.\right.
$$

Since (30) holds, the terms $Q_{\sigma^{\prime}, \sigma^{*^{\prime}}}$ are non negative for all $D_{\sigma^{\prime}, \sigma^{*^{\prime}}} \in \mathfrak{D}_{e x t}$ and consequently

$$
\mathcal{A}_{K} \geqslant \frac{\left|D_{\sigma, \sigma^{*}}\right|}{|K|} \rho_{\sigma, \sigma^{*}} Q_{\sigma, \sigma^{*}}
$$


Thus, $T_{K}$ is non negative as soon as

$$
Q_{\sigma, \sigma^{*}}-c\left(e_{\sigma, \sigma^{*}}\right) \frac{\delta t}{\sqrt{2} \gamma} \frac{|\sigma||K|}{\left|D_{\sigma, \sigma^{*}}\right|^{2}} \geqslant 0
$$

or equivalently, using the expression of $Q_{\sigma, \sigma^{*}}$,

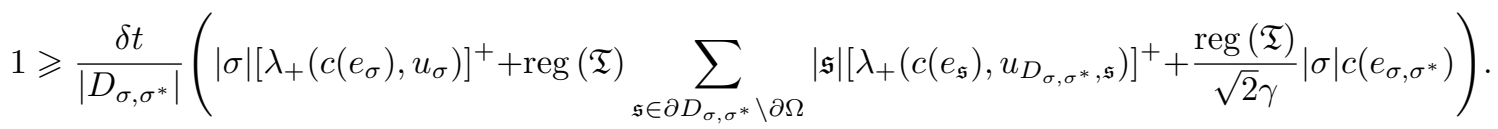

This inequality holds when assumption (39) is fulfilled. The same result obviously holds for $T_{L}$. Concerning $T_{K^{*}}$ (and $T_{L^{*}}$ ), we obtain a slightly different condition. Indeed, owing to (35), we can write

$$
T_{K^{*}} \geqslant \frac{\left\|\overline{\mathbf{u}}_{K^{*}}-\mathbf{u}_{K^{*}}\right\|^{2}}{4\left|D_{\sigma, \sigma^{*}}\right|}\left(\left|D_{\sigma, \sigma^{*}} \cap K^{*}\right| \mathcal{A}_{K^{*}}-\rho_{\sigma, \sigma^{*}} c\left(e_{\sigma, \sigma^{*}}\right) \frac{\delta t\left|\sigma^{*}\right|}{\sqrt{2} \gamma}\right) .
$$

Going back to (37) and since (30) holds, we have $\mathcal{A}_{K^{*}} \geqslant \frac{\left|D_{\sigma, \sigma^{*}} \cap K^{*}\right|}{\left|K^{*}\right|} \rho_{\sigma, \sigma^{*}} Q_{\sigma, \sigma^{*}}^{*}$ where

$$
\begin{aligned}
Q_{\sigma, \sigma^{*}}^{*} \geqslant 1-\frac{\delta t}{\left|D_{\sigma, \sigma^{*}}\right|} \frac{|\sigma|}{2}\left((1+\operatorname{reg}(\mathfrak{T})) \frac{\mathcal{F}_{\sigma}^{+}}{\rho_{\sigma, \sigma^{*}}}\right. & \left.-\operatorname{reg}(\mathfrak{T}) \frac{\mathcal{F}_{\sigma}^{-}}{\rho_{\sigma, \sigma^{*}}}\right) \\
& -\frac{\delta t}{\left|D_{\sigma, \sigma^{*}}\right|} \operatorname{reg}(\mathfrak{T}) \sum_{\mathfrak{s} \in \partial D_{\sigma, \sigma^{*} \backslash \partial \Omega}}|\mathfrak{s}| \frac{\mathcal{F}_{D_{\sigma, \sigma^{*}, \mathfrak{s}}}^{+}}{\rho_{\sigma, \sigma^{*}}} .
\end{aligned}
$$

Thus, we obtain that $T_{K^{*}}$ is non negative as soon as

$$
\begin{aligned}
1 \geqslant \frac{\delta t}{\left|D_{\sigma, \sigma^{*}}\right|}\left(\frac{|\sigma|}{2}((1\right. & \left.+\operatorname{reg}(\mathfrak{T})) \frac{\mathcal{F}_{\sigma}^{+}}{\rho_{\sigma, \sigma^{*}}}-\operatorname{reg}(\mathfrak{T}) \frac{\mathcal{F}_{\sigma}^{-}}{\rho_{\sigma, \sigma^{*}}}\right) \\
& \left.+\operatorname{reg}(\mathfrak{T}) \sum_{\mathfrak{s} \in \partial D_{\sigma, \sigma^{*} \backslash \partial \Omega}}|\mathfrak{s}| \frac{\mathcal{F}_{D_{\sigma, \sigma^{*}, \mathfrak{s}}}^{+}}{\rho_{\sigma, \sigma^{*}}}+c\left(e_{\sigma, \sigma^{*}}\right) \frac{\left|\sigma^{*}\right|}{\sqrt{2} \gamma} \operatorname{reg}(\mathfrak{T})^{3}\right)
\end{aligned}
$$

We conclude that $T_{K^{*}}$ and $T_{L^{*}}$ are non negative if the following condition holds

$$
\begin{aligned}
& 1 \geqslant \frac{\delta t}{\left|D_{\sigma, \sigma^{*}}\right|}\left(\frac{|\sigma|}{2}\left((1+\operatorname{reg}(\mathfrak{T}))\left[\lambda_{+}\left(c\left(e_{\sigma}\right), u_{\sigma}\right)\right]^{+}+\operatorname{reg}(\mathfrak{T}) \frac{\rho_{\text {Dir }}}{\rho_{\sigma, \sigma^{*}}}\left[\lambda_{-}\left(c\left(e_{\text {Dir }}\right), u_{\text {Dir }}\right)\right]^{-}\right)\right.
\end{aligned}
$$

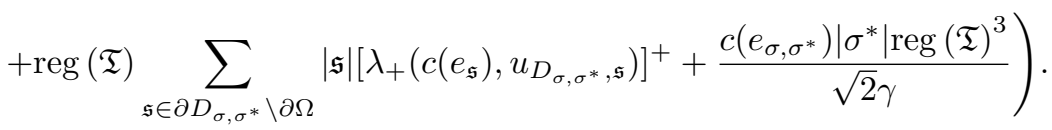

This last inequality is implied by assumption (39). This concludes the proof.

\section{Conservation of total energy}

Definition 4.1. We define a kinetic energy $E_{\sigma, \sigma^{*}}^{\mathrm{kin}}$, stored on the cell $D_{\sigma, \sigma^{*}} \in \mathfrak{D}_{\text {int }}$, by the formula

$$
E_{\sigma, \sigma^{*}}^{\mathrm{kin}}=\frac{\left|D_{\sigma, \sigma^{*}} \cap K\right| \frac{\rho_{K}\left\|\mathbf{u}_{K}\right\|^{2}}{2}+\left|D_{\sigma, \sigma^{*}} \cap L\right| \frac{\rho_{L}\left\|\mathbf{u}_{L}\right\|^{2}}{2}+\left|D_{\sigma, \sigma^{*}} \cap K^{*}\right| \frac{\rho_{K^{*}}\left\|\mathbf{u}_{K^{*}}\right\|^{2}}{2}+\left|D_{\sigma, \sigma^{*}} \cap L^{*}\right| \frac{\rho_{L^{*}}\left\|\mathbf{u}_{L^{*}}\right\|^{2}}{2}}{2\left|D_{\sigma, \sigma^{*}}\right| \rho_{\sigma, \sigma^{*}}}
$$

and a total energy $E_{\sigma, \sigma^{*}}$, stored on the cell $D_{\sigma, \sigma^{*}} \in \mathfrak{D}_{\text {int }}$, by setting

$$
E_{\sigma, \sigma^{*}}=e_{\sigma, \sigma^{*}}+E_{\sigma, \sigma^{*}}^{\mathrm{kin}}
$$


We wish to write a local conservation equation for the total energy $\rho_{\sigma, \sigma^{*}} E_{\sigma, \sigma^{*}}$. This property is in fact related to the duality relations between discrete operators, as discussed in [23], and based on Lemma 3.3 .

Proposition 4.2. The discrete total energy $\rho_{\sigma, \sigma^{*}} E_{\sigma, \sigma^{*}}$ satisfies the following conservative equation on $\mathfrak{D}_{\text {int }}$ :

$$
\frac{\bar{\rho}_{\sigma, \sigma^{*}} \bar{E}_{\sigma, \sigma^{*}}-\rho_{\sigma, \sigma^{*}} E_{\sigma, \sigma^{*}}}{\delta t}+\frac{1}{\left|D_{\sigma, \sigma^{*}}\right|} \sum_{\mathfrak{s} \in \partial D_{\sigma, \sigma^{*}}}|\mathfrak{s}| \mathcal{T}_{D_{\sigma, \sigma^{*}, \mathfrak{s}}}+\frac{1}{\left|D_{\sigma, \sigma^{*}}\right|} \sum_{\mathfrak{s} \in \partial D_{\sigma, \sigma^{*}}}|\mathfrak{s}| q_{D_{\sigma, \sigma^{*}, \mathfrak{s}}}=0,
$$

where

- $\mathcal{T}_{D_{\sigma, \sigma^{*}, \mathfrak{s}}}$ is a conservative total energy flux through the interface $\mathfrak{s}$ of the diamond cell $D_{\sigma, \sigma^{*}}$,

- $\frac{1}{\left|D_{\sigma, \sigma^{*}}\right|} \sum_{\mathfrak{s} \in \partial D_{\sigma, \sigma^{*}}}|\mathfrak{s}| q_{D_{\sigma, \sigma^{*}, \mathfrak{s}}}$ is a conservative discrete version of $\boldsymbol{\nabla} \cdot(p \overline{\mathbf{u}})$ on the diamond cell $D_{\sigma, \sigma^{*}}$.

Proof. We identify the corresponding fluxes by using Lemma 3.3. It is helpful to bear in mind the typical shape of a diamond cell with vertices $x_{K}, x_{K^{*}}, x_{L}, x_{L^{*}}$ as depicted in Fig. 3 . Let $X \in\left\{K, L, K^{*}, L^{*}\right\}$ and multiply the kinetic energy balance equation on the cell $X$ by $\frac{\left|D_{\sigma, \sigma^{*}} \cap X\right|}{2\left|D_{\sigma, \sigma^{*}}\right|}$. Next, add the four relations to the equation for the internal energy (27) on the cell $D_{\sigma, \sigma^{*}}$.

*) The first task is to identify some conservative fluxes $\mathcal{K}_{D_{\sigma, \sigma^{*}, \mathfrak{F}}}$ such that

$$
\begin{aligned}
\sum_{\mathfrak{s} \in \partial D_{\sigma, \sigma^{*}}}|\mathfrak{s}| \mathcal{K}_{D_{\sigma, \sigma^{*}, \mathfrak{s}}} & =\frac{\left|D_{\sigma, \sigma^{*}} \cap K\right|}{2|K|} \sum_{\sigma^{\prime} \in \partial K}\left|\sigma^{\prime}\right| \mathcal{K}_{K, \sigma^{\prime}}+\frac{\left|D_{\sigma, \sigma^{*}} \cap L\right|}{2|L|} \sum_{\sigma^{\prime} \in \partial L}\left|\sigma^{\prime}\right| \mathcal{K}_{L, \sigma^{\prime}} \\
& +\frac{\left|D_{\sigma, \sigma^{*}} \cap K^{*}\right|}{2\left|K^{*}\right|} \sum_{\sigma^{\prime} \in \partial K^{*}}\left|\sigma^{\prime}\right| \mathcal{K}_{K^{*}, \sigma^{\prime}}+\frac{\left|D_{\sigma, \sigma^{*}} \cap L^{*}\right|}{2\left|L^{*}\right|} \sum_{\sigma^{\prime} \in \partial L^{*}}\left|\sigma^{\prime}\right| \mathcal{K}_{L^{*}, \sigma^{\prime}}
\end{aligned}
$$

Applying Lemma 3.3, on each primal cell $\mathscr{C}=K$ with $\mathcal{X}_{K, \sigma}=\mathcal{K}_{K, \sigma}$ provides a function $\omega_{K}$ which satisfies (11) and (12). For the dual mesh, we proceed as for the primal mesh to define $\omega_{K^{*}}$ from the fluxes $\mathcal{X}_{K^{*}, \sigma^{*}}=\mathcal{K}_{K^{*}, \sigma^{*}}$ on the interfaces of $K^{*}$. We next define a conservative flux of kinetic energy for each $\mathfrak{s}=\left[x_{K}, x_{K^{*}}\right] \in \partial D_{\sigma, \sigma^{*}}$ as follows

$$
\mathcal{K}_{D_{\sigma, \sigma^{*}, \mathfrak{s}}}=\frac{1}{2|\mathfrak{s}|} \int_{\mathfrak{s}}\left(\omega_{K}+\omega_{K^{*}}\right) \mathbf{n}_{D_{\sigma, \sigma^{*}, \mathfrak{s}}} \text { where } \mathfrak{s}=\mathfrak{s}_{K K^{*}}
$$

With a convenient reorganization of the terms, we write $\sum_{\mathfrak{s} \in \partial D_{\sigma, \sigma^{*}}}|\mathfrak{s}| \mathcal{K}_{D_{\sigma, \sigma^{*}, \mathfrak{s}} \text { as }}$

$$
\begin{aligned}
& \frac{1}{2}\left(\int_{\mathfrak{s}_{K K^{*}}} \omega_{K} \mathbf{n}_{D_{\sigma, \sigma^{*}, \mathfrak{s}_{K} K^{*}}}+\int_{\mathfrak{s}_{K L^{*}}} \omega_{K} \mathbf{n}_{D_{\sigma, \sigma^{*}, \mathfrak{s}_{K L^{*}}}}\right) \\
& +\frac{1}{2}\left(\int_{\mathfrak{s}_{L K^{*}}} \omega_{L} \mathbf{n}_{D_{\sigma, \sigma^{*}, \mathfrak{s}_{L K^{*}}}}+\int_{\mathfrak{s}_{L L^{*}}} \omega_{L} \mathbf{n}_{D_{\sigma, \sigma^{*}, \mathfrak{s}_{L L}}}\right) \\
& +\frac{1}{2}\left(\int_{\mathfrak{s}_{K K^{*}}} \omega_{K^{*}} \mathbf{n}_{D_{\sigma, \sigma^{*}, \mathfrak{s}_{K} K^{*}}}+\int_{\mathfrak{s}_{L K^{*}}} \omega_{K^{*}} \mathbf{n}_{D_{\sigma, \sigma^{*}, \mathfrak{s}_{L} K^{*}}}\right) \\
& +\frac{1}{2}\left(\int_{\mathfrak{s}_{K L^{*}}} \omega_{L^{*}} \mathbf{n}_{D_{\sigma, \sigma^{*}, \mathfrak{s}_{K L^{*}}}}+\int_{\mathfrak{s}_{L L^{*}}} \omega_{L^{*}} \mathbf{n}_{D_{\sigma, \sigma^{*}, \mathfrak{s}_{L L^{*}}}}\right) .
\end{aligned}
$$

Since the kinetic energy fluxes $\mathcal{K}_{K, \sigma}$ and $\mathcal{K}_{K^{*}, \sigma^{*}}$ are conservative, we have

$$
\mathcal{K}_{K, \sigma}+\mathcal{K}_{L, \sigma}=0 \text { and } \mathcal{K}_{K^{*}, \sigma^{*}}+\mathcal{K}_{L^{*}, \sigma^{*}}=0 .
$$


Applying again (12) in Lemma 3.3, these two equalities recast as

$$
\frac{1}{2}\left(\int_{\sigma} \omega_{K} \mathbf{n}_{K, \sigma}+\int_{\sigma} \omega_{L} \mathbf{n}_{L, \sigma}\right)=0
$$

and

$$
\frac{1}{2}\left(\int_{\sigma^{*}} \omega_{K^{*}} \mathbf{n}_{K^{*}, \sigma^{*}}+\int_{\sigma^{*}} \omega_{L^{*}} \mathbf{n}_{L^{*}, \sigma^{*}}\right)=0 .
$$

We add these expressions in the sum $\sum_{\mathfrak{s} \in \partial D_{\sigma, \sigma^{*}}}|\mathfrak{s}| \mathcal{K}_{D_{\sigma, \sigma^{*}, \mathfrak{s}}}$ and we get

$$
\begin{aligned}
& \sum_{\mathfrak{s} \in \partial D_{\sigma, \sigma^{*}}}|\mathfrak{s}| \mathcal{K}_{D_{\sigma, \sigma^{*}, \mathfrak{s}}}=\frac{1}{2}\left(\int_{\sigma} \omega_{K} \mathbf{n}_{K, \sigma}+\int_{\mathfrak{s}_{K K^{*}}} \omega_{K} \mathbf{n}_{D_{\sigma, \sigma^{*}, \mathfrak{s} K K^{*}}}+\int_{\mathfrak{s}_{K L^{*}}} \omega_{K} \mathbf{n}_{D_{\sigma, \sigma^{*}, \mathfrak{s}_{K L}}}\right) \\
& +\frac{1}{2}\left(\int_{\sigma} \omega_{L} \mathbf{n}_{L, \sigma}+\int_{\mathfrak{s}_{L K^{*}}} \omega_{L} \mathbf{n}_{D_{\sigma, \sigma^{*}, \mathfrak{s}_{L K^{*}}}}+\int_{\mathfrak{s}_{L L^{*}}} \omega_{L} \mathbf{n}_{D_{\sigma, \sigma^{*}, \mathfrak{s}_{L L}}}\right) \\
& +\frac{1}{2}\left(\int_{\sigma^{*}} \omega_{K^{*}} \mathbf{n}_{K^{*}, \sigma^{*}}+\int_{\mathfrak{s}_{K K^{*}}} \omega_{K^{*}} \mathbf{n}_{D_{\sigma, \sigma^{*}, \mathfrak{s}_{K K^{*}}}}+\int_{\mathfrak{s}_{L K^{*}}} \omega_{K^{*}} \mathbf{n}_{D_{\sigma, \sigma^{*}, \mathfrak{s}_{L K^{*}}}}\right) \\
& +\frac{1}{2}\left(\int_{\sigma^{*}} \omega_{L^{*}} \mathbf{n}_{L^{*}, \sigma^{*}}+\int_{\mathfrak{s}_{K L^{*}}} \omega_{L^{*}} \mathbf{n}_{D_{\sigma, \sigma^{*}, \mathfrak{s}_{K L^{*}}}}+\int_{\mathfrak{s}_{L L^{*}}} \omega_{L^{*}} \mathbf{n}_{D_{\sigma, \sigma^{*}, \mathfrak{s}_{L L^{*}}}}\right) .
\end{aligned}
$$

Thus, by the divergence theorem, and because $\nabla \cdot \omega_{X}$ is constant over the cell $X$, we get

$$
\begin{aligned}
\sum_{\mathfrak{s} \in \partial D_{\sigma, \sigma^{*}}}|\mathfrak{s}| \mathcal{K}_{D_{\sigma, \sigma^{*}, \mathfrak{s}}} & =\frac{\left|D_{\sigma, \sigma^{*}} \cap K\right|}{2} \nabla \cdot \omega_{K}+\frac{\left|D_{\sigma, \sigma^{*}} \cap L\right|}{2} \nabla \cdot \omega_{L} \\
& +\frac{\left|D_{\sigma, \sigma^{*}} \cap K^{*}\right|}{2} \nabla \cdot \omega_{K^{*}}+\frac{\left|D_{\sigma, \sigma^{*}} \cap L^{*}\right|}{2} \nabla \cdot \omega_{L^{*}}
\end{aligned}
$$

Applying (11) in Lemma 3.3 shows that (42) is satisfied. Finally, we define a conservative flux of total energy $\mathcal{T}_{D_{\sigma, \sigma^{*}, \mathfrak{s}}}$ through the interface $\mathfrak{s}$ of the diamond cell $D_{\sigma, \sigma^{*}}$ by

$$
\mathcal{T}_{D_{\sigma, \sigma^{*}, \mathfrak{s}}}=\mathcal{K}_{D_{\sigma, \sigma^{*}, \mathfrak{s}}}+\mathcal{E}_{D_{\sigma, \sigma^{*}, \mathfrak{s}}}
$$

**) We now turn to the pressure term. There are four terms coming from the sum of the kinetic energy equations and the discrete version of $p \boldsymbol{\nabla} \cdot \mathbf{u}$, namely

$$
\begin{aligned}
\frac{\left|D_{\sigma, \sigma^{*}} \cap K\right|}{2} \overline{\mathbf{u}}_{K} & \cdot\left(\boldsymbol{\nabla}_{\boldsymbol{d}} p\right)_{K}+\frac{\left|D_{\sigma, \sigma^{*}} \cap L\right|}{2} \overline{\mathbf{u}}_{L} \cdot\left(\boldsymbol{\nabla}_{\boldsymbol{d}} p\right)_{L} \\
+ & \frac{\left|D_{\sigma, \sigma^{*}} \cap K^{*}\right|}{2} \overline{\mathbf{u}}_{K^{*}} \cdot\left(\boldsymbol{\nabla}_{\boldsymbol{d}} p\right)_{K^{*}}+\frac{\left|D_{\sigma, \sigma^{*}} \cap L^{*}\right|}{2} \overline{\mathbf{u}}_{L^{*}} \cdot\left(\boldsymbol{\nabla}_{\boldsymbol{d}} p\right)_{L^{*}} \\
& +\left|D_{\sigma, \sigma^{*}}\right| p_{\sigma, \sigma^{*}}\left(\boldsymbol{\nabla}_{\boldsymbol{d}} \cdot \mathbf{u}\right)_{\sigma, \sigma^{*}} .
\end{aligned}
$$

We wish to rewrite this sum as $\sum_{\mathfrak{s} \in \partial D_{\sigma, \sigma^{*}}}|\mathfrak{s}| q_{D_{\sigma, \sigma^{*}, \mathfrak{s}}}$ with $q_{D_{\sigma, \sigma^{*}, \mathfrak{s}}}$ verifying the conservation property $q_{D_{\sigma, \sigma^{*}, \mathfrak{s}}}=-q_{D_{\sigma^{\prime}, *^{*}, \mathfrak{s}}}$ where $\mathfrak{s}=D_{\sigma, \sigma^{*}} \mid D_{\sigma^{\prime}, \sigma^{*}}$.

To this end, we apply Lemma 3.3 again on each primal (resp. dual) cell $\mathscr{C}=K$ (resp. $K^{*}$ ) with, now, $\mathcal{X}_{K, \sigma}=p_{\sigma, \sigma^{*}} \overline{\mathbf{u}}_{K} \cdot \mathbf{n}_{K, \sigma}$ (resp. $\mathcal{X}_{K^{*}, \sigma^{*}}=p_{\sigma, \sigma^{*}} \overline{\mathbf{u}}_{K^{*}} \cdot \mathbf{n}_{K^{*}, \sigma^{*}}$. It provides functions $\omega_{K}$ (resp. $\omega_{K^{*}}$ ) that satisfy (11) and (12).

We next define, for each $\mathfrak{s}=\left[x_{K}, x_{K^{*}}\right] \in \partial D_{\sigma, \sigma^{*}}$,

$$
q_{D_{\sigma, \sigma^{*}, \mathfrak{s}}}=\frac{1}{2|\mathfrak{s}|} \int_{\mathfrak{s}}\left(\omega_{K}+\omega_{K^{*}}\right) \cdot \mathbf{n}_{D_{\sigma, \sigma^{*}, \mathfrak{s}}} \text { where } \mathfrak{s}=\mathfrak{s}_{K K^{*}} .
$$

By construction, this quantity is conservative.

We are now going to check that the sum $\sum_{\mathfrak{s} \in \partial D_{\sigma, \sigma^{*}}}|\mathfrak{s}| q_{D_{\sigma, \sigma^{*}, \mathfrak{s}}}$ coincides with (45). With a 
convenient reorganization of the terms, we write $\sum_{\mathfrak{s} \in \partial D_{\sigma, \sigma^{*}}}|\mathfrak{s}| q_{D_{\sigma, \sigma^{*}, \mathfrak{s}}}$ as

$$
\begin{aligned}
& \frac{1}{2}\left(\int_{\mathfrak{s}_{K K^{*}}} \omega_{K} \cdot \mathbf{n}_{\sigma, \mathfrak{s}_{K K^{*}}}+\int_{\mathfrak{s}_{K L^{*}}} \omega_{K} \cdot \mathbf{n}_{\sigma, \mathfrak{s}_{K L^{*}}}\right)+\frac{1}{2}\left(\int_{\mathfrak{s}_{L K^{*}}} \omega_{L} \cdot \mathbf{n}_{\sigma, \mathfrak{s}_{L} K^{*}}+\int_{\mathfrak{s}_{L L^{*}}} \omega_{L} \cdot \mathbf{n}_{\sigma, \mathfrak{s}_{L L^{*}}}\right) \\
& +\frac{1}{2}\left(\int_{\mathfrak{s}_{K K^{*}}} \omega_{K^{*}} \cdot \mathbf{n}_{\sigma, \mathfrak{s} K K^{*}}+\int_{\mathfrak{s}_{L K^{*}}} \omega_{K^{*}} \cdot \mathbf{n}_{\sigma, \mathfrak{s}_{L} K^{*}}\right)+\frac{1}{2}\left(\int_{\mathfrak{s}_{K L^{*}}} \omega_{L^{*}} \cdot \mathbf{n}_{\sigma, \mathfrak{s} K L^{*}}+\int_{\mathfrak{s}_{L L^{*}}} \omega_{L^{*}} \cdot \mathbf{n}_{\sigma, \mathfrak{s}_{L L}}\right) .
\end{aligned}
$$

We make use again of Lemma 3.3 to write

$$
\int_{\sigma} \omega_{K} \cdot \mathbf{n}_{K, \sigma}=|\sigma| p_{\sigma, \sigma^{*}} \overline{\mathbf{u}}_{K} \cdot \mathbf{n}_{K, \sigma} \text { and } \int_{\sigma^{*}} \omega_{K^{*}} \cdot \mathbf{n}_{K^{*}, \sigma^{*}}=\left|\sigma^{*}\right| p_{\sigma, \sigma^{*}} \overline{\mathbf{u}}_{K^{*}} \cdot \mathbf{n}_{K^{*}, \sigma^{*}}
$$

For $X \in\{K, L\}$ we add $\frac{1}{2} \int_{\sigma} \omega_{X} \cdot \mathbf{n}_{X, \sigma}+\frac{1}{2} \int_{\sigma^{*}} \omega_{X^{*}} \cdot \mathbf{n}_{X^{*}, \sigma^{*}}$ and substract $\frac{|\sigma|}{2} p_{\sigma, \sigma^{*}} \overline{\mathbf{u}}_{X} \cdot \mathbf{n}_{X, \sigma}+$ $\frac{\left|\sigma^{*}\right|}{2} p_{\sigma, \sigma^{*}} \overline{\mathbf{u}}_{X^{*}} \cdot \mathbf{n}_{X^{*}, \sigma^{*}}$ in the above expression and we get

$$
\begin{aligned}
& \sum_{\mathfrak{s} \in \partial D_{\sigma, \sigma^{*}}}|\mathfrak{s}| q_{D_{\sigma, \sigma^{*}, \mathfrak{s}}}=\frac{1}{2}\left(\int_{\sigma} \omega_{K} \cdot \mathbf{n}_{K, \sigma}+\int_{\mathfrak{s}_{K K^{*}}} \omega_{K} \cdot \mathbf{n}_{\sigma, \mathfrak{s}_{K K^{*}}}+\int_{\mathfrak{s}_{K L^{*}}} \omega_{K} \cdot \mathbf{n}_{\sigma, \mathfrak{s}_{K L^{*}}}\right) \\
& +\frac{1}{2}\left(\int_{\sigma} \omega_{L} \cdot \mathbf{n}_{L, \sigma}+\int_{\mathfrak{s}_{L K^{*}}} \omega_{L} \cdot \mathbf{n}_{\sigma, \mathfrak{s}_{L K}}+\int_{\mathfrak{s}_{L L^{*}}} \omega_{L} \cdot \mathbf{n}_{\sigma, \mathfrak{s}_{L L^{*}}}\right)
\end{aligned}
$$

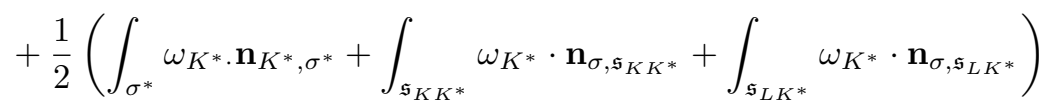

$$
\begin{aligned}
& +\frac{1}{2}\left(\int_{\sigma^{*}} \omega_{L^{*}} \cdot \mathbf{n}_{L^{*}, \sigma^{*}}+\int_{\mathfrak{s}_{K L^{*}}} \omega_{L^{*}} \cdot \mathbf{n}_{\sigma, \mathfrak{s}_{K L^{*}}}+\int_{\mathfrak{s}_{L L^{*}}} \omega_{L^{*}} \cdot \mathbf{n}_{\sigma, \mathfrak{s}_{L L^{*}}}\right) \\
& -\frac{|\sigma|}{2} p_{\sigma, \sigma^{*}}\left(\overline{\mathbf{u}}_{K} \cdot \mathbf{n}_{K, \sigma}+\overline{\mathbf{u}}_{L} \cdot \mathbf{n}_{L, \sigma}\right)-\frac{\left|\sigma^{*}\right|}{2} p_{\sigma, \sigma^{*}}\left(\overline{\mathbf{u}}_{K^{*}} \cdot \mathbf{n}_{K^{*}, \sigma^{*}}+\overline{\mathbf{u}}_{L^{*}} \cdot \mathbf{n}_{L^{*}, \sigma^{*}}\right) .
\end{aligned}
$$

Thus, using equation (11) of Lemma 3.3 together with Definition 3.11 of the discrete divergence operator yields

$$
\begin{aligned}
\sum_{\mathfrak{s} \in \partial D_{\sigma, \sigma^{*}}}|\mathfrak{s}| q_{D_{\sigma, \sigma^{*}, \mathfrak{s}}=} & \frac{\left|D_{\sigma, \sigma^{*}} \cap K\right|}{2} \nabla \cdot \omega_{K}+\frac{\left|D_{\sigma, \sigma^{*}} \cap L\right|}{2} \nabla \cdot \omega_{L} \\
& +\frac{\left|D_{\sigma, \sigma^{*}} \cap K^{*}\right|}{2} \nabla \cdot \omega_{K^{*}}+\frac{\left|D_{\sigma, \sigma^{*}} \cap L^{*}\right|}{2} \nabla \cdot \omega_{L^{*}} \\
& +\left|D_{\sigma, \sigma^{*}}\right| p_{\sigma, \sigma^{*}}\left(\nabla_{\boldsymbol{d}} \cdot \overline{\mathbf{u}}\right)_{\sigma, \sigma^{*}}
\end{aligned}
$$

Applying (12) in Lemma 3.3 shows that

$$
\begin{aligned}
\sum_{\mathfrak{s} \in \partial D_{\sigma, \sigma^{*}}}|\mathfrak{s}| q_{D_{\sigma, \sigma^{*}, \mathfrak{s}}}= & \frac{\left|D_{\sigma, \sigma^{*}} \cap K\right|}{2|K|} \sum_{\sigma^{\prime} \in \partial K}\left|\sigma^{\prime}\right| q_{K, \sigma^{\prime}}+\frac{\left|D_{\sigma, \sigma^{*}} \cap L\right|}{2|L|} \sum_{\sigma^{\prime} \in \partial L}\left|\sigma^{\prime}\right| q_{L, \sigma^{\prime}} \\
= & \frac{\left|D_{\sigma, \sigma^{*}} \cap K^{*}\right|}{2\left|K^{*}\right|} \sum_{\sigma^{*^{\prime}} \in \partial K^{*}}\left|\sigma^{*^{\prime}}\right| q_{K^{*}, \sigma^{*^{\prime}}}+\frac{\left|D_{\sigma, \sigma^{*}} \cap L^{*}\right|}{2\left|L^{*}\right|} \sum_{\sigma^{*^{\prime} \in \partial L^{*}}}\left|\sigma^{*^{\prime}}\right| q_{L^{*}, \sigma^{*^{\prime}}} \\
& +\left|D_{\sigma, \sigma^{*}}\right| p_{\sigma, \sigma^{*}}\left(\boldsymbol{\nabla}_{\boldsymbol{d}} \cdot \overline{\mathbf{u}}\right)_{\sigma, \sigma^{*}} .
\end{aligned}
$$

Finally, coming back to the definition of $q_{K, \sigma}$ and next, to Definition 3.7 of the discrete pressure gradient, we remark that for $X \in\left\{K, L, K^{*}, L^{*}\right\}$

$$
\frac{1}{|X|} \sum_{\sigma^{\prime} \in \partial X}\left|\sigma^{\prime}\right| q_{X, \sigma^{\prime}}=\frac{1}{|X|} \sum_{\sigma^{\prime} \in \partial X}\left|\sigma^{\prime}\right| p_{\sigma^{\prime}, \sigma^{*}} \overline{\mathbf{u}}_{X} \cdot \mathbf{n}_{X, \sigma^{\prime}}=\overline{\mathbf{u}}_{X} \cdot\left(\nabla_{\boldsymbol{d}} p\right)_{X}
$$


We can conclude that

$$
\begin{aligned}
& \sum_{\mathfrak{s} \in \partial D_{\sigma, \sigma^{*}}}|\mathfrak{s}| q_{D_{\sigma, \sigma^{*}, \mathfrak{s}}}=\frac{\left|D_{\sigma, \sigma^{*}} \cap K\right|}{2} \overline{\mathbf{u}}_{K} \cdot\left(\nabla_{\boldsymbol{d}} p\right)_{K}+\frac{\left|D_{\sigma, \sigma^{*}} \cap L\right|}{2} \overline{\mathbf{u}}_{L} \cdot\left(\boldsymbol{\nabla}_{\boldsymbol{d}} p\right)_{L} \\
& +\frac{\left|D_{\sigma, \sigma^{*}} \cap K^{*}\right|}{2} \overline{\mathbf{u}}_{K^{*}} \cdot\left(\nabla_{\boldsymbol{d}} p\right)_{K^{*}}+\frac{\left|D_{\sigma, \sigma^{*}} \cap L^{*}\right|}{2} \overline{\mathbf{u}}_{L^{*}} \cdot\left(\boldsymbol{\nabla}_{\boldsymbol{d}} p\right)_{L^{*}} \\
& +\left|D_{\sigma, \sigma^{*}}\right| p_{\sigma, \sigma^{*}}\left(\boldsymbol{\nabla}_{\boldsymbol{d}} \cdot \overline{\mathbf{u}}\right)_{\sigma, \sigma^{*}} .
\end{aligned}
$$

While the numerical strategy is based on staggered discretization and makes use of the internal energy equation, obtaining a discrete conservative equation on a quantity which is consistent with the total energy is a remarkable fact. It opens doors to check the convergence of the scheme in the spirit of Lax-Wendroff's analysis [40] — which is far from obvious since the standard notion of flux-consistency does not make sense on staggered grids; we refer the reader to [2] and $[19,31]$ for developments in this direction. The derivation of entropy inequalities is a much more delicate issue, which requires further efforts.

\section{$5 \quad$ Numerical simulations}

In this Section we present some numerical test cases on unstructured grids. We compare the performance of the scheme to the MAC discretization [25]. The unstructured primal meshes used in this Section are tessellations made of triangles, provided by GMSH, which leads to a dual mesh the cells of which are polygons of any type.

For these meshes, the coefficient reg $(\mathfrak{T})$ takes values around 10 . We define the characteristic length $h$ of the different meshes as follows

$$
h=\max \left(|\sigma|,\left|\sigma^{*}\right|\right) .
$$

\subsection{Consistency analysis with a 2D manufactured solution}

In order to numerically validate the scheme, we compute the solution of the 2D problem

$$
\left\{\begin{array}{l}
\partial_{t} \rho+\nabla \cdot(\rho \mathbf{u})=0, \\
\partial_{t}(\rho \mathbf{u})+\nabla \cdot(\rho \mathbf{u} \otimes \mathbf{u})+\nabla p=f(t, \mathbf{x}), \\
\partial_{t}(\rho e)+\nabla \cdot(\rho e \mathbf{u})+p \boldsymbol{\nabla} \cdot \mathbf{u}=0
\end{array}\right.
$$

where the force field $(t, \mathbf{x}) \mapsto f(t, \mathbf{x})$ is tailored so that the smooth solution reads

$$
\left\{\begin{array}{l}
\rho^{\mathrm{ex}}(t, \mathbf{x})=\exp \left(-2 \sqrt{(x \cos (t)+y \sin (t)+1)^{2}+(-x \sin (t)+y \cos (t)-0.1)^{2}}\right), \\
u^{\mathrm{ex}}(t, \mathbf{x})=-y, \\
v^{\operatorname{ex}}(t, \mathbf{x})=x, \\
e^{\operatorname{ex}}(t, \mathbf{x})=\frac{\exp \left(-3 \sqrt{(x \cos (t)+y \sin (t)+1)^{2}+(-x \sin (t)+y \cos (t)+0.1)^{2}}\right)}{\rho(t, \mathbf{x})},
\end{array}\right.
$$

with $\mathbf{x}=(x, y)$. We perform the simulations for $t \in[0,0.2]$ with $\gamma=1.4$ on the circle of center $(0,0)$ and radius 2 . We use a series of tessellations made of triangles, provided by GMSH: the characteristic length used in GMSH (the quantity that determines the mesh size) is divided by 2 between each mesh. 
The discrete $L^{2}$ norms of the errors between the discrete and the exact solutions, for the density, the internal energy and the first component of the velocity, on the different meshes, are reported in Table 1 and Table 2:

$$
\begin{aligned}
& \mathfrak{e}_{2, \rho}=\left(\sum_{\substack{D_{\sigma, \sigma^{*}} \in \mathfrak{D} \\
\sigma^{*}=\left[\mathbf{x}_{\left.K^{*}, \mathbf{x}_{L^{*}}\right]}\right.}}\left|D_{\sigma, \sigma^{*}}\right|\left|\rho_{D_{\sigma, \sigma^{*}}}-\rho^{\mathrm{ex}}\left(\frac{\mathbf{x}_{K^{*}}+\mathbf{x}_{L^{*}}}{2}\right)\right|^{2}\right)^{\frac{1}{2}}, \\
& \mathfrak{e}_{2, e}=\left(\sum_{\substack{D_{\sigma, \sigma^{*}} \in \mathfrak{D} \\
\sigma^{*}=\left[\mathbf{x}_{\left.K^{*}, \mathbf{x}_{L^{*}}\right]}\right.}}\left|D_{\sigma, \sigma^{*}}\right|\left|e_{D_{\sigma, \sigma^{*}}}-e^{\operatorname{ex}}\left(\frac{\mathbf{x}_{K^{*}}+\mathbf{x}_{L^{*}}}{2}\right)\right|^{2}\right)^{\frac{1}{2}}, \\
& \mathfrak{e}_{2, u}=\left(\sum_{K \in \mathfrak{M}}|K|\left|u_{K}-u^{\mathrm{ex}}\left(\mathbf{x}_{K}\right)\right|^{2}\right)^{\frac{1}{2}}, \quad \mathfrak{e}_{2, v}=\left(\sum_{K \in \mathfrak{M}}|K|\left|v_{K}-v^{\mathrm{ex}}\left(\mathbf{x}_{K}\right)\right|^{2}\right)^{\frac{1}{2}}, \\
& \mathfrak{e}_{2, u^{*}}=\left(\sum_{K \in \mathfrak{M}^{*} \cup \partial \mathfrak{M}^{*}}\left|K^{*}\right|\left|u_{K^{*}}-u^{\mathrm{ex}}\left(\mathbf{x}_{K^{*}}\right)\right|^{2}\right)^{\frac{1}{2}}, \quad \mathfrak{e}_{2, v^{*}}=\left(\sum_{K \in \mathfrak{M}^{*} \cup \partial \mathfrak{M}^{*}}\left|K^{*}\right|\left|v_{K^{*}}-v^{\mathrm{ex}}\left(\mathbf{x}_{K^{*}}\right)\right|^{2}\right)^{\frac{1}{2}} .
\end{aligned}
$$

We also report the characteristic length $h$ of the different meshes. The results are almost the same for the second component of the velocities. For this test case, we have set $\delta t=10^{-4}$ : the small value of the time step ensures that the stability condition is satisfied for all the considered grids. It appears that it corresponds to the following condition at the initial time:

$$
\max _{D_{\sigma, \sigma^{*}} \in \mathfrak{D}_{\text {int }}}\left(\frac { \delta t } { | D _ { \sigma , \sigma ^ { * } } | } \sum _ { \mathfrak { s } \in \partial D _ { \sigma , \sigma ^ { * } } } | \mathfrak { s } | \left[\lambda_{+}\left(c\left(e_{\mathfrak{s}}\right), u_{\left.\left.D_{\sigma, \sigma^{*}, \mathfrak{s}}\right)\right]^{+}}\right)=\frac{1}{4}\right.\right.
$$

We use Dirichlet boundary conditions. We observe as expected a first order convergence. In Fig. 4 we show the numerical solutions (density and the internal energy) obtained on the finest mesh at time $t=0$ and $t=0.2$.

\begin{tabular}{|c||c||c|c||c|c||}
\hline$i$ & $h^{(i)}$ & $\mathfrak{e}_{2, \rho}^{(i)}$ & $\frac{\log \left(\mathfrak{e}_{2, \rho}^{(i)} / \mathfrak{e}_{2, \rho}^{(i-1)}\right)}{\log \left(h^{(i)} / h^{(i-1)}\right)}$ & $\mathfrak{e}_{2, e}^{(i)}$ & $\frac{\log \left(\mathfrak{e}_{2, e}^{(i)} / \mathfrak{e}_{2, e}^{(i-1)}\right)}{\log \left(h^{(i)} / h^{(i-1)}\right)}$ \\
\hline \hline 1 & $2.62 \times 10^{-1}$ & $4.07 \times 10^{-2}$ & -- & $1.71 \times 10^{-1}$ & -- \\
\hline 2 & $1.43 \times 10^{-1}$ & $2.49 \times 10^{-2}$ & 0.82 & $9.28 \times 10^{-2}$ & 1.02 \\
\hline 3 & $7.10 \times 10^{-2}$ & $1.40 \times 10^{-2}$ & 0.82 & $4.87 \times 10^{-2}$ & 0.92 \\
\hline 4 & $3.74 \times 10^{-2}$ & $8.04 \times 10^{-3}$ & 0.87 & $2.54 \times 10^{-2}$ & 1.01 \\
\hline 5 & $1.96 \times 10^{-2}$ & $4.45 \times 10^{-3}$ & 0.91 & $1.31 \times 10^{-2}$ & 1.02 \\
\hline 6 & $1.01 \times 10^{-2}$ & $2.36 \times 10^{-3}$ & 0.96 & $6.74 \times 10^{-3}$ & 1.01 \\
\hline
\end{tabular}

Table 1: Error in $L^{2}$-norm between approximate and exact solutions for the density and the internal energy on several meshes. 


\begin{tabular}{|c||c|c||c|c||c|}
\hline$i$ & $h^{(i)}$ & $\mathfrak{e}_{2, u}^{(i)}$ & $\frac{\log \left(\mathfrak{e}_{2, u}^{(i)} / \mathfrak{e}_{2, u}^{(i-1)}\right)}{\log \left(h^{(i)} / h^{(i-1)}\right)}$ & $\mathfrak{e}_{2, u^{*}}^{(i)}$ & $\frac{\log \left(\mathfrak{e}_{2, u^{*}}^{(i)} / \mathfrak{e}_{2, u^{*}}^{(i-1)}\right)}{\log \left(h^{(i)} / h^{(i-1)}\right)}$ \\
\hline \hline 1 & $2.62 \times 10^{-1}$ & $1.12 \times 10^{-1}$ & -- & $3.01 \times 10^{-1}$ & -- \\
\hline 2 & $1.43 \times 10^{-1}$ & $6.19 \times 10^{-2}$ & 0.98 & $1.67 \times 10^{-1}$ & 0.97 \\
\hline 3 & $7.10 \times 10^{-2}$ & $3.36 \times 10^{-2}$ & 0.87 & $9.40 \times 10^{-2}$ & 0.82 \\
\hline 4 & $3.74 \times 10^{-2}$ & $1.80 \times 10^{-2}$ & 0.97 & $5.27 \times 10^{-2}$ & 0.90 \\
\hline 5 & $1.96 \times 10^{-2}$ & $9.56 \times 10^{-3}$ & 0.98 & $2.89 \times 10^{-2}$ & 0.93 \\
\hline 6 & $1.01 \times 10^{-2}$ & $4.94 \times 10^{-3}$ & 1.00 & $1.57 \times 10^{-2}$ & 0.92 \\
\hline
\end{tabular}

Table 2: Error in $L^{2}$-norm between approximate and exact solutions for the first component of the velocity on several meshes.

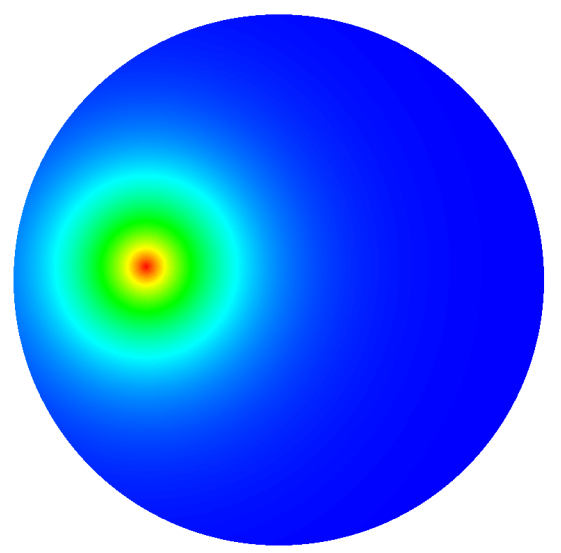

(a) Density, $t=0$

$(\max =1.01, \min =2.43 \mathrm{e}-3)$

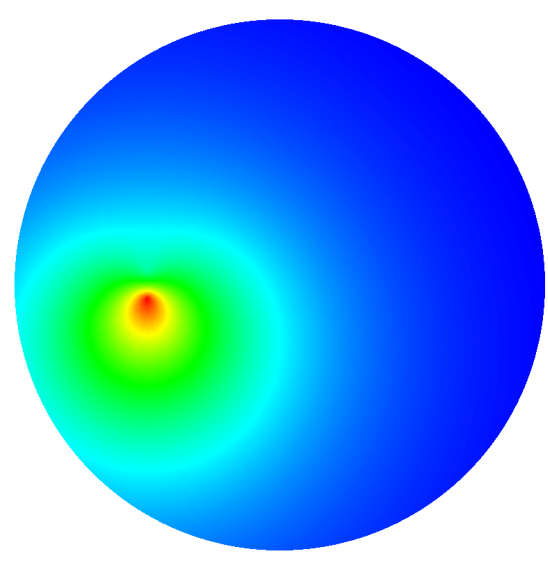

(c) Internal energy, $t=0$

$(\max =1.49, \min =4.52 \mathrm{e}-2)$

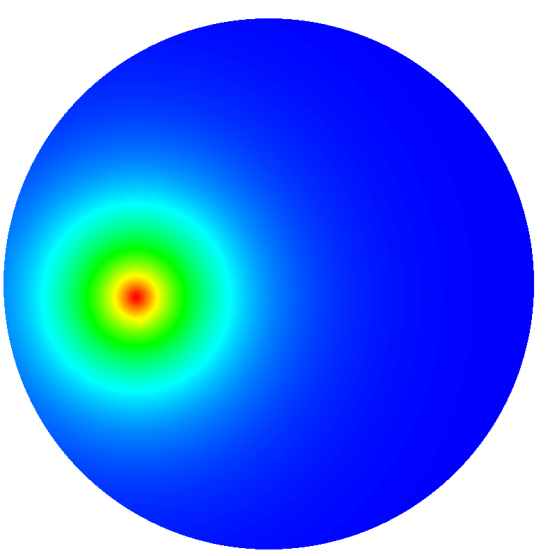

(b) Density, $t=0.2$

$(\max =0.96, \min =2.47 \mathrm{e}-3)$

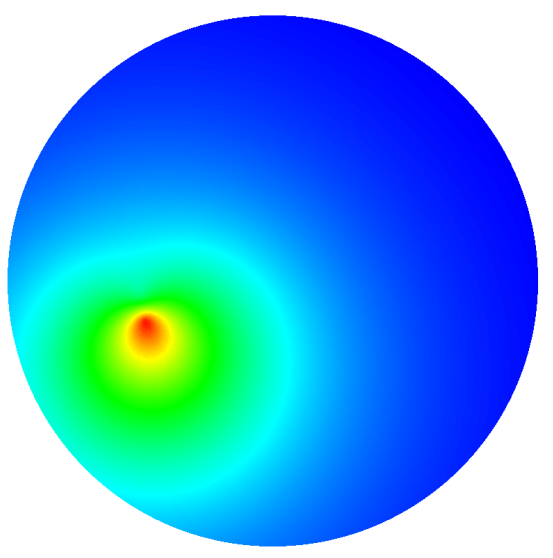

(d) Internal energy, $t=0.2$

$(\max =1.46, \min =4.67 \mathrm{e}-2)$

Figure 4: Density and internal energy, numerical solution on a mesh with 921772 primal cells. 


\subsection{Simulations of 1d Riemann problems}

We now check the ability of the scheme in finding non smooth solutions. To this end, we run the 2D code, on a 2D unstructured grid, but with Riemann data constant in the second direction $y$. We thus expect to preserve the 1D geometry of the solution, given by solving the 1D Riemann problem. The mesh size is $h=6.9 \times 10^{-4}$. The mesh contains about 5 cells in the $y$ direction and 21305 cells in total. The time step is $\delta t=2 \times 10^{-6}$. Beyond the geometric robustness, dealing with such discontinuous solutions it is also important to check whether or not the scheme is able to capture the expected solution, without developing non entropic singularities. In the staggered framework where the equations are treated "separately", the order in which the unknowns are updated might matter. The difficulty is illustrated in [34] on standard Sod's problems [50], showing differences between the updates "density/internal energy/velocity" and "density/velocity/internal energy", the latter (which is the order used here) producing non entropic shocks, see [34, Fig. 23]. The 1D simulations performed in [25] on the same test case shows that working with the numerical fluxes introduced in Definition 3.1 restores the expected entropic solution, see [34, Fig. 19]. This is confirmed by the experiments performed with the 2D unstructured code: Fig. 5 compares the exact solution of the Riemann problem and the numerical solution for the initial data $\rho_{l}=\rho_{r}=1, u_{l}=u_{r}=0 p_{l}=1000$, $p_{r}=0.001, T=0.012$, with the adiabatic exponent $\gamma=1.4$. Contrarily to the results of [34], the rarefaction wave does not contain non admissible shocks and the results are similar to those obtained in the purely 1D simulations of [25].

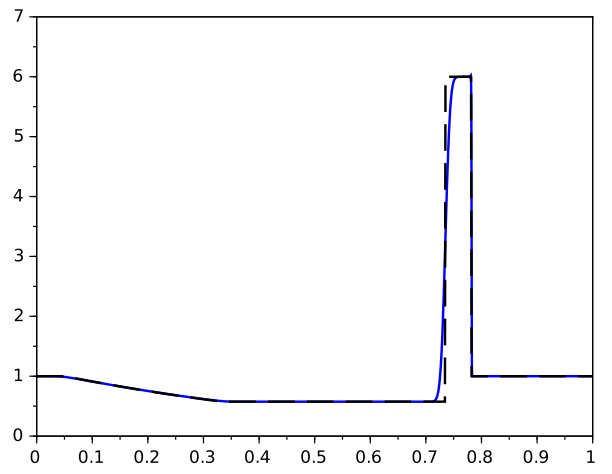

(a) Density $\rho$

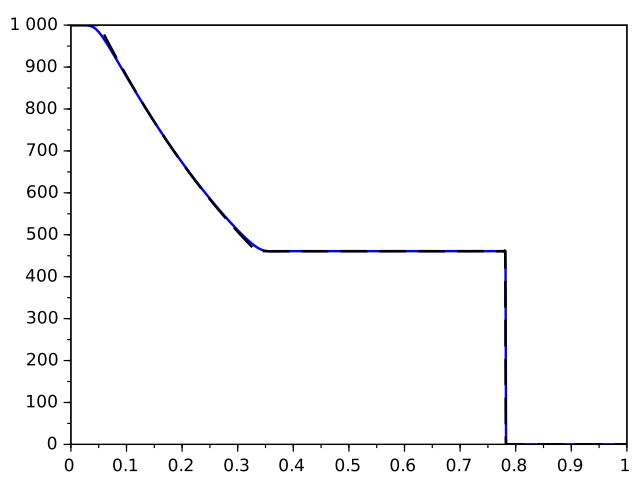

(c) Pressure $p$

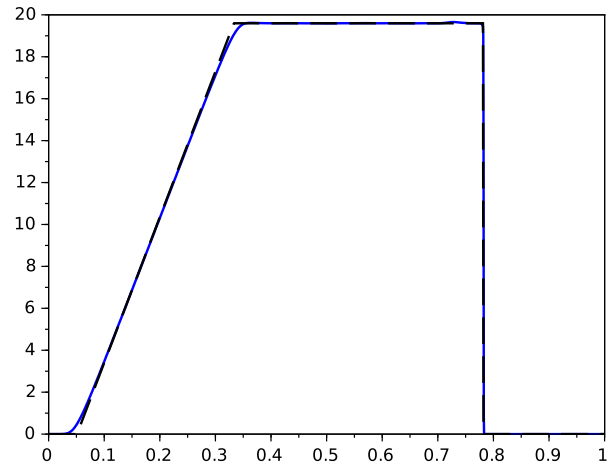

(b) Velocity (horizontal component)

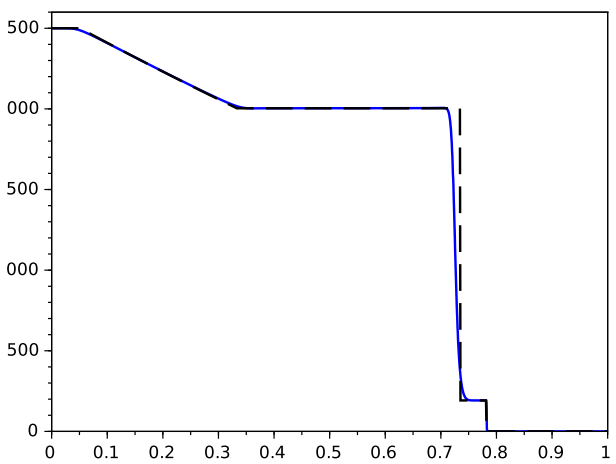

(d) Internal energy $e$

Figure 5: Riemann problem, horizontal cutlines $(y=0)$ at time $T=0.012$. Exact solution (black dotted line) and numerical solutions (blue solid line), $h=6.9 \times 10^{-4}, \delta t=2 \times 10^{-6}$. 


\subsection{Simulations of $2 \mathrm{~d}$ singular solutions}

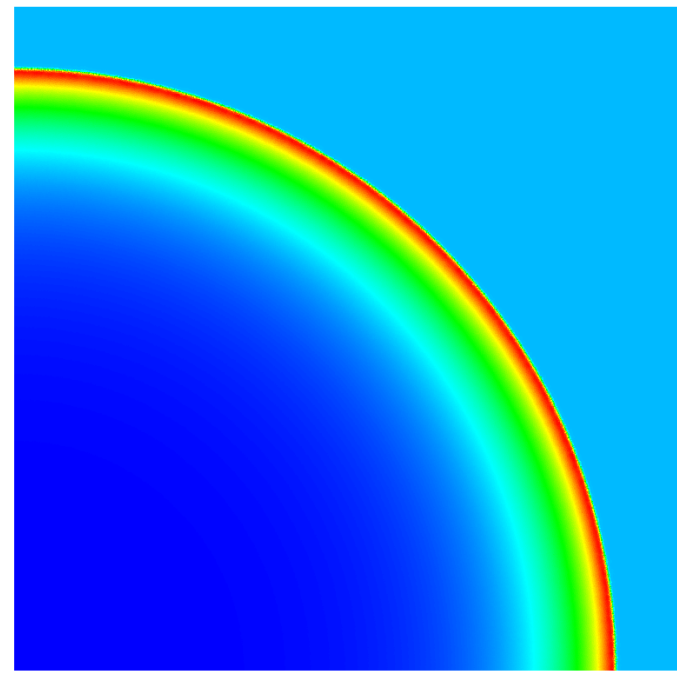

(a) Density

$(\min =0.0023, \max =5.44)$

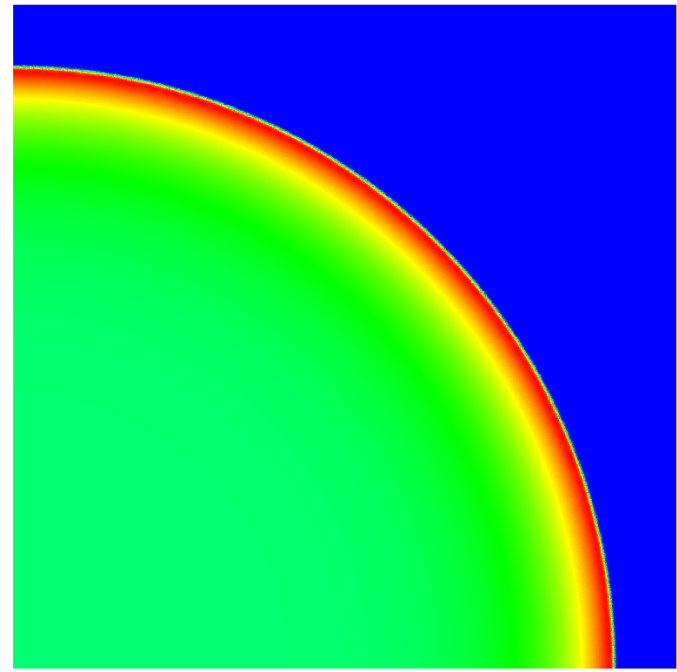

(b) Pressure

$(\min =0.00, \max =0.50)$

Figure 6: Sedov blast wave problem, density and pressure at time $T=0.4$ on an unstructured triangular grid with 341136 primal cells $\left(h=3.02 \times 10^{-3}\right)$ and $\delta t=10^{-6}$.

1. Sedov blast wave problem. This test case [49] gives rise to a diverging radially symmetric shock wave $[12,26,46]$. Initially the gas has constant state $\rho=1, \mathbf{u}=0$, and the pressure is 0 , except at the origin where the total energy is concentrated. The analytical solution is explicitly known [36]. To approximate this solution, we start from a constant pressure in the set $\mathcal{C}$ of the diamond cells that contain the origin, with an initial energy $E_{0}=1$. Since, initially, the velocity is zero and $\rho=1$, it means that we set

$$
p_{D}=(\gamma-1) \frac{E_{0}}{|\mathcal{C}|}, \quad \forall D \in \mathcal{C} \quad \text { and } \quad p_{D}=0, \quad \forall D \notin \mathcal{C},
$$

at the initial time. Thanks to the symmetry of the solution, it is possible to only perform the simulations on the quarter plane. In this case, to take into account the symmetry, $|\mathcal{C}|$ is replaced by $4|\mathcal{C}|$ in the initialisation of the pressure. We perform three computations with three different triangular meshes respectively with 21502, 85376 and 341136 primal cells. The corresponding mesh sizes and the time steps we used are the following: $(h=$ $\left.1.17 \times 10^{-2}, \delta t=2 \times 10^{-5}\right),\left(h=5.93 \times 10^{-3}, \delta t=2 \times 10^{-6}\right)$ and $\left(h=3.02 \times 10^{-3}\right.$, $\left.\delta t=10^{-6}\right)$. Fig. 6 shows the discrete density and pressure at time $T=0.4$ on the finest mesh. It shows the expansion of the blast wave: the symmetry of the solution is well preserved. We see in Fig. 7 radial cutlines of the exact and the numerical solutions at $T=0.2$ and $T=0.4$ for the three different meshes. The solution is well captured with an improved accuracy as the mesh is refined and the expansion speed is correct.

We also check on this test case the conservation properties of the scheme. The total mass $\sum_{D_{\sigma, \sigma^{*}} \in \mathfrak{D}}\left|D_{\sigma, \sigma^{*}}\right| \rho_{\sigma, \sigma^{*}}$ is preserved up to machine precision. The discrete integral of the averaged total energy (see definition 4.1)

$$
\sum_{D_{\sigma, \sigma^{*} \in \mathfrak{D}}}\left|D_{\sigma, \sigma^{*}}\right| \rho_{\sigma, \sigma^{*}} E_{\sigma, \sigma^{*}}
$$


is also preserved up to machine precision. The discrete total momentum ( computed on both primal and dual meshes)

$$
\sum_{K \in \mathfrak{T}}|K| \rho_{K} \mathbf{u}_{K} \quad \text { and } \quad \sum_{K^{*} \in \mathfrak{T}}\left|K^{*}\right| \rho_{K^{*}} \mathbf{u}_{K^{*}}
$$

are also preserved up to the machine precision when the simulation is perfomed on the full domain. On the quarter plane, the pressure term in the momentum equation brings a non-vanishing contribution on the boundary.

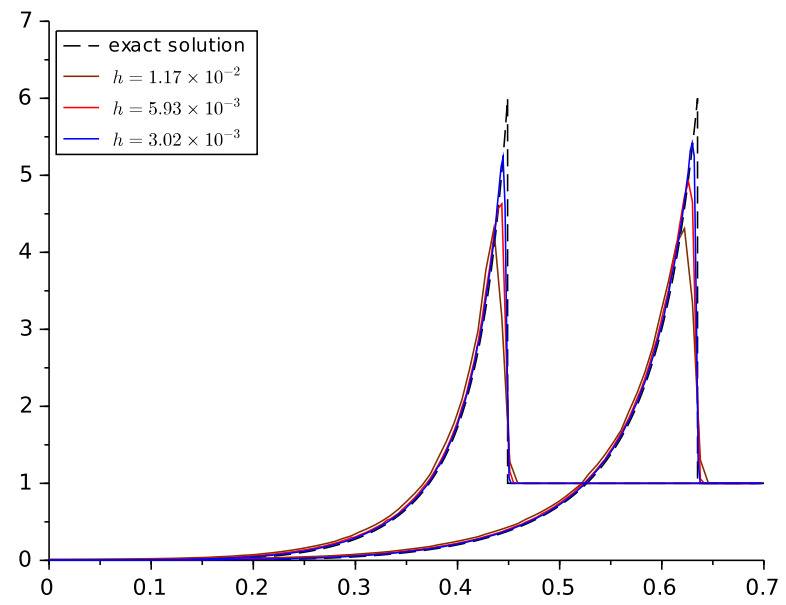

(a) Density

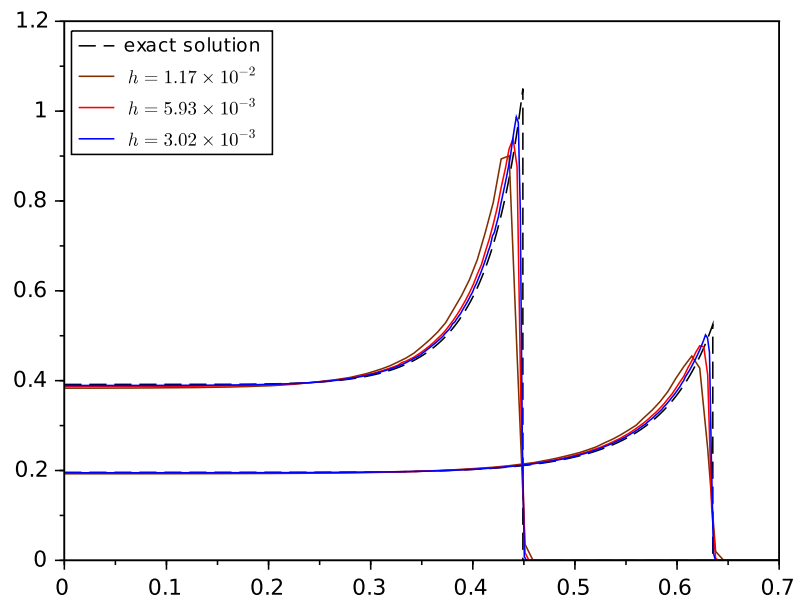

(b) Pressure

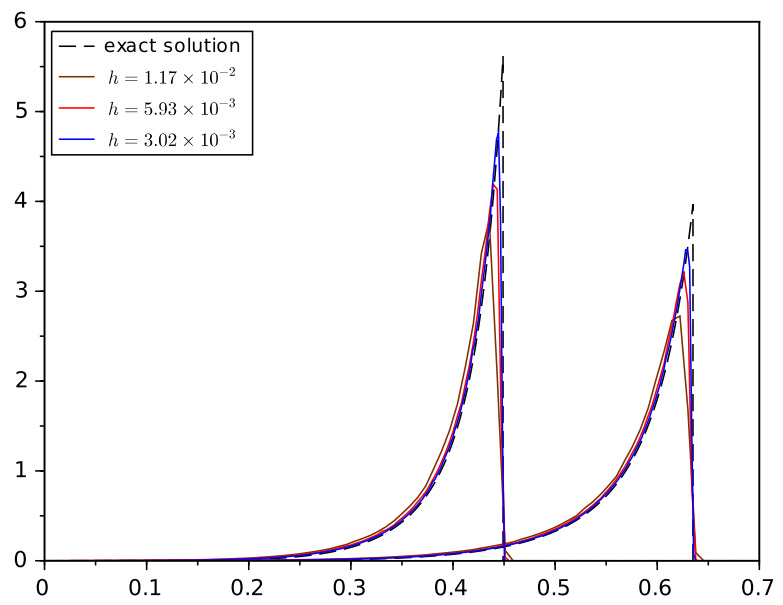

(c) Momentum

Figure 7: Sedov blast wave problem, radial cutlines at time $T=0.2$ and $T=0.4$. Exact solution and numerical solutions on three different meshes.

2. Mach 3 Wind Tunnel. We use the scheme for the simulation of the 2D Mach 3 wind tunnel with a step. The computational domain $\Omega$ is the L-shaped domain

$$
\Omega=\Omega_{0} \backslash \Omega_{\text {step }}, \quad \Omega_{0}=[0,3] \times[0,1], \quad \Omega_{\text {step }}=[0.6,3] \times[0,0.2] .
$$

We perform the simulation for $t \in[0,4]$ with $\gamma=1.4$ and $\delta t=10^{-4}$. The initial data reads $\rho=1.4, \mathbf{u}=(3,0)$ and $p=1$. On the top and bottom walls, we use reflection boundary 
conditions which means zero flux boundary conditions. To make the flow enter through the left boundary we use a Dirichlet boundary condition, $\rho=1.4, p=1$ and $\mathbf{u}=(3,0)$, whereas a free boundary condition is used for the right section.

In Fig. 8 (at the top), we present the result obtained at $T=4$ with an unstructured primal mesh made of 204254 triangles and 307081 edges. We compare this result to the one obtained in [25] on a $960 \times 320$ cartesian grid which is reported in Fig. 8 (at the bottom).
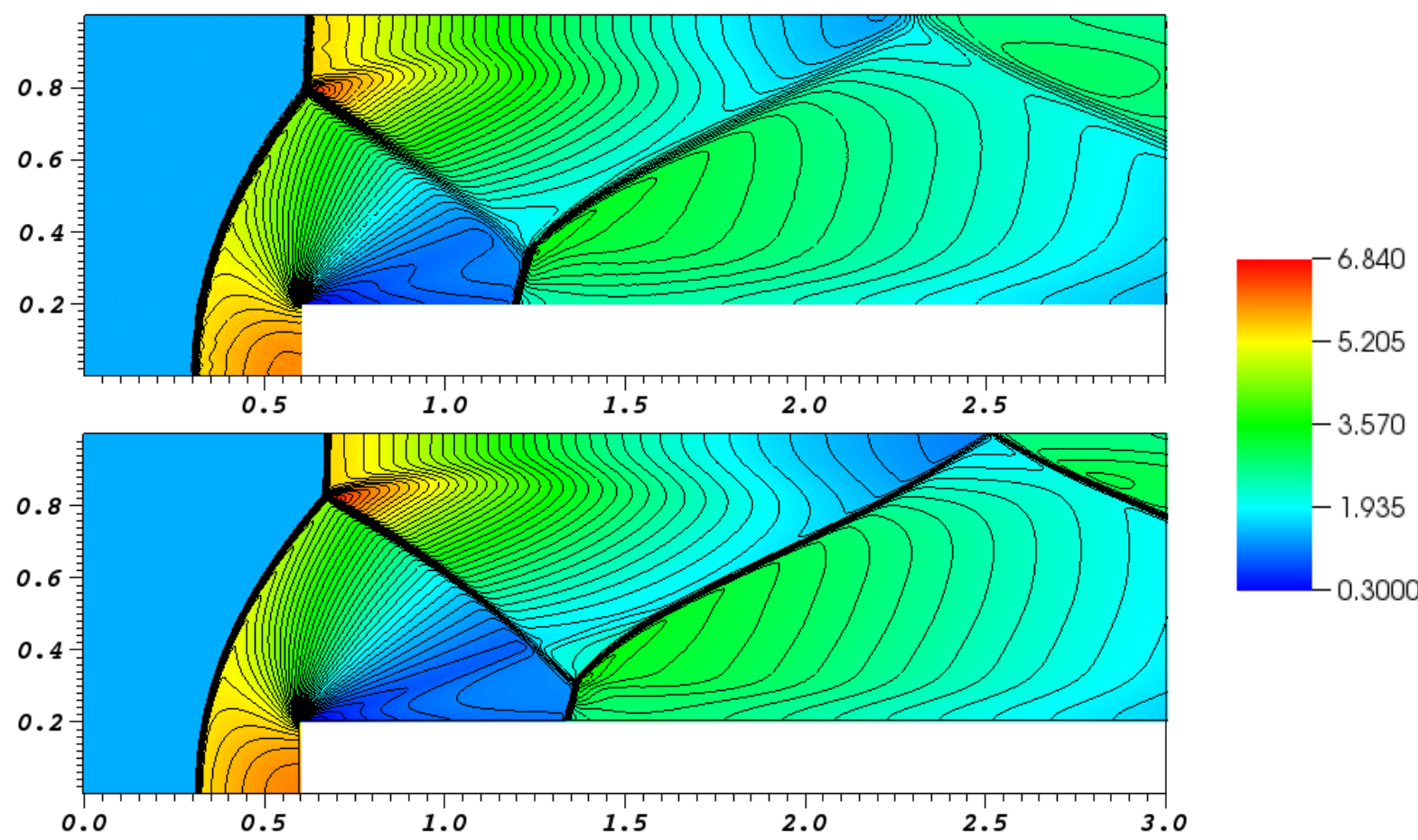

Figure 8: Simulation of the 2D Mach 3 wind tunnel with a step, density with 50 contour lines on a MAC mesh at bottom $(\min =0.30, \max =6.53)$ and on an unstructured triangular grid at top $(\min =0.33, \max =6.84)$ at $T=4$.

3. Falling water columns. The next test case is inspired from [3]: we consider the 2D simulation of three falling columns into a rectangular basin. The computational domain is the two-dimensional square $[-1,1] \times[-1,1]$. We deal with the full Euler system with $\gamma=2$. Initially we suppose a constant initial temperature (or internal energy $e$ ) in the basin. The PDE system is endowed with zero flux boundary conditions and the following initial data

$$
\left\{\begin{array}{l}
\rho(0, \mathbf{x})=3+\mathbb{1}_{(x-0.5)^{2}+(y-0.5)^{2}<(0.15)^{2}}+\mathbb{1}_{(x+0.5)^{2}+(y+0.5)^{2}<(0.15)^{2}}+2 \cdot \mathbb{1}_{x^{2}+y^{2}<(0.2)^{2}} \\
e(0, \mathbf{x})=1 \\
u(0, \mathbf{x})=0 \\
v(0, \mathbf{x})=0
\end{array}\right.
$$

with $\mathbf{x}=(x, y)$. In Fig. 9 and 10, we show the density and the internal energy at time $T=1.035$ with $\delta t=10^{-4}$. The mesh has 43400 primal cells made of triangles and 65356 edges. The result is compared with the same simulation made on a $255 \times 255$ Cartesian grid with the MAC scheme presented in [25]. 

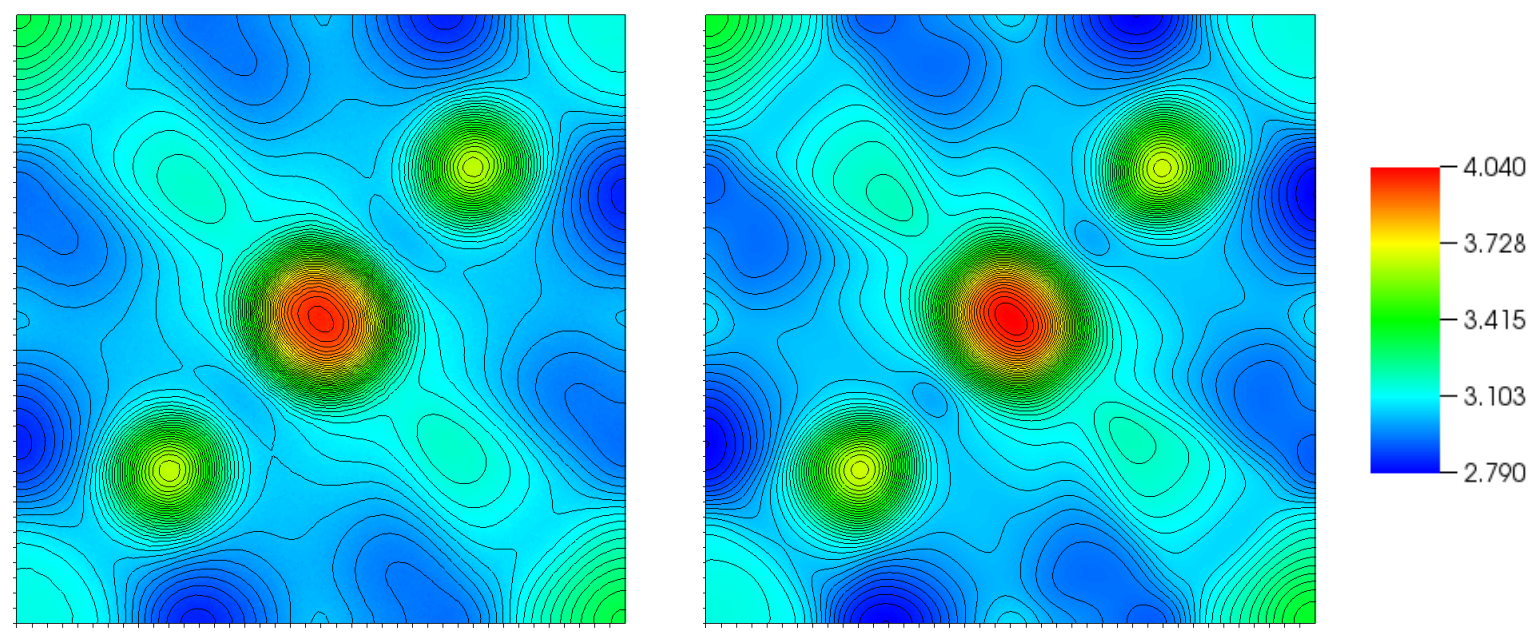

Figure 9: Simulation of the 2D three falling columns into a rectangular basin: Density with 50 contour lines on an unstructured grid at left $(\min =2.83$, $\max =4.01)$ and a $255 \times 255$ Cartesian mesh at right $(\min =2.79, \max =4.04)$ at $T=1.035$.
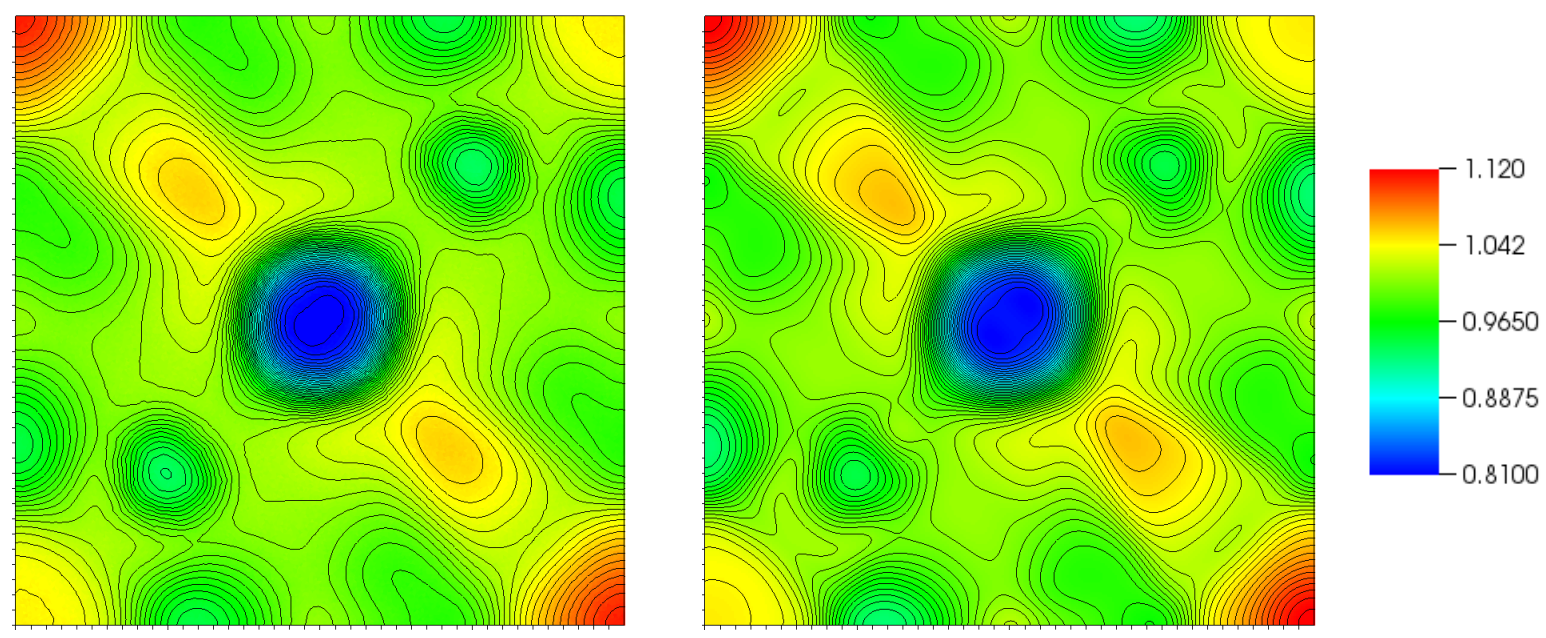

Figure 10: Simulation of the 2D three falling columns into a rectangular basin: Internal energy with 50 contour lines on an unstructured grid at left $(\min =0.81, \max =1.11)$ and on a $255 \times 255$ Cartesian mesh at right $(\min =0.81, \max =1.12)$ at $T=1.035$.

4. Flow past a circular bump. The last test case is a 2D supersonic flow in a channel with a circular arc bump. The fluid flows from left to right. Due to the geometry of the obstacle, the MAC grid proposed in [25] is not appropriate. The computational domain $\Omega$ is the rectangle $[0,3] \times[0,1]$ with a circular arc bump of length 1 and thickness 0.04 located at the bottom, at a distance 1 from the inlet. We perform the simulation up to the final time $T=5$. We have set $\gamma=1.4$ and the time step is $\delta t=10^{-4}$. The initial data are given by $\rho=1, \mathbf{u}=(1.65,0)$ and $p=1$, so that the initial Mach number is 1.65 . On the top and bottom walls, we use zero flux boundary conditions and on the left we use Dirichlet boundary condition $\rho=1, \mathbf{u}=(1.65,0)$ and $p=1$ whereas on the right boundary a free boundary condition is used. In Fig. 11, we show the Mach Number with 50 contour lines. 


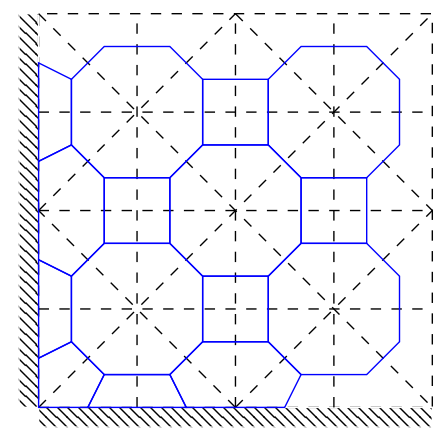

(a) Direct dual mesh
IIIIIII $\partial \Omega$

- Dual mesh

Primal mesh

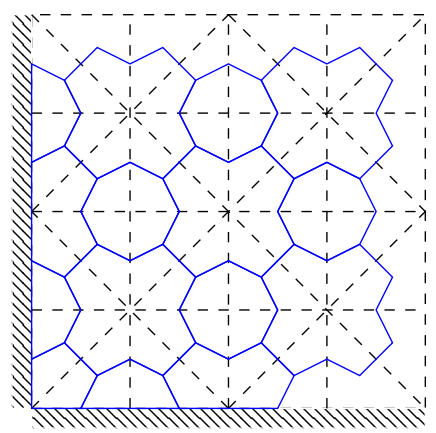

(b) Barycentric dual mesh

Figure 12: Structured triangular mesh

The simulation is performed on a triangular mesh with 516192 primal cells and 775313 edges. Oblique shocks are formed at the two extremities of the bump. All the shocks are well resolved. The simulation can be compared with the result presented in [51].

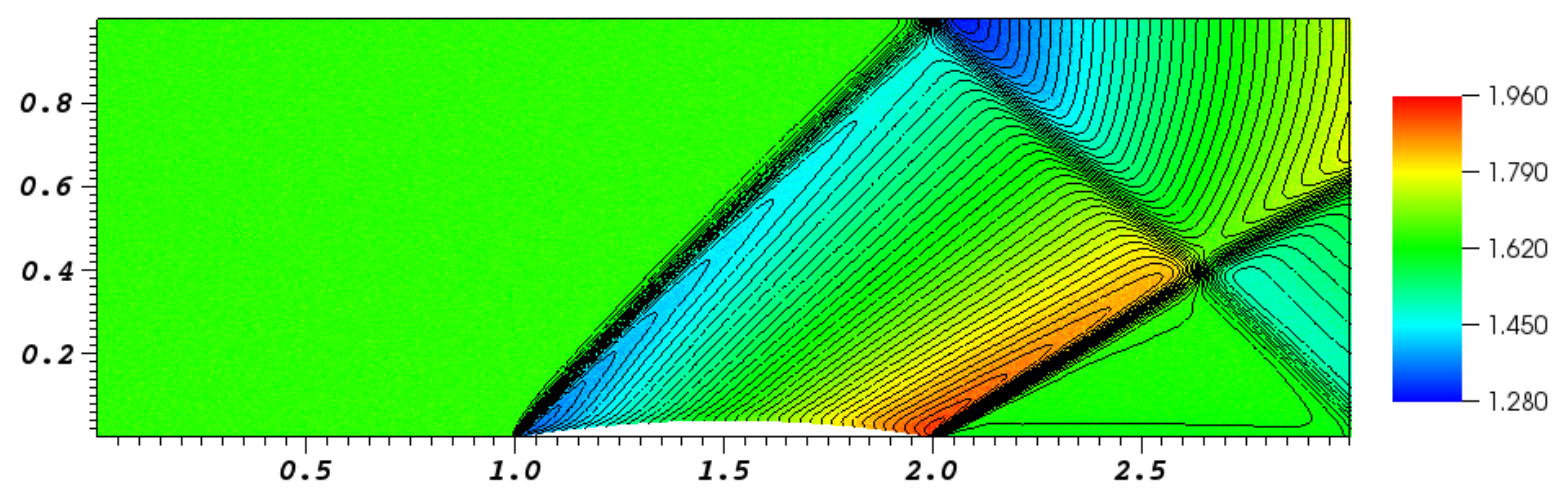

Figure 11: Simulation of the 2D supersonic flow in a channel with a circular arc bump flows from left to right: Mach number $(\min =1.28, \max =1.96)$ at time $T=5$ with 50 contour lines on an unstructured mesh.

\section{A Barycentric mesh}

An alternative construction of the dual mesh is obtained by joining the centers $x_{K}$ to the midpoints of the edges of the primal mesh: this is the barycentric mesh [13, 14]. Fig. 12 provides an example of the direct and the barycentric approaches, starting from the same structured triangular primal mesh. Clearly, the barycentric dual mesh can have a much more complicated structure than the direct dual mesh, requiring more involved descriptors in the code, but in practice it can be more robust with respect to mesh orientation effects and stability issues, see for instance $[10,11]$.

It might happen that the direct dual mesh produces non convex diamonds cells, in which case the edges of the dual mesh are not included in the diamond. This is illustrated in Fig. 13. For such complicated geometries, it is preferable to work with the barycentric dual mesh. In this situation, considering a diamond cell $D_{\sigma, \sigma^{*}}, \sigma=\left[x_{K^{*}}, x_{L^{*}}\right]$ is still included in the diamond, and there are two edges of the dual mesh, hereafter denoted $\sigma_{K}^{*}$ and $\sigma_{L}^{*}$, which belong to this diamond, while possibly $\sigma^{*}=\left[x_{K}, x_{L}\right] \not \subset D_{\sigma, \sigma^{*}}$, see Fig. 13. For the barycentric mesh, the 
definition of $\mathfrak{D}_{K^{*}}$ should be adapted: it becomes $\mathfrak{D}_{K^{*}}=\left\{D_{\sigma, \sigma^{*}} \in \mathfrak{D}, \partial K^{*} \cap D_{\sigma, \sigma^{*}} \neq \emptyset\right\}$. All statements of the paper apply to this framework; we are going to discuss the slight adaptations of the arguments, which still relies on the construction derived in [23].

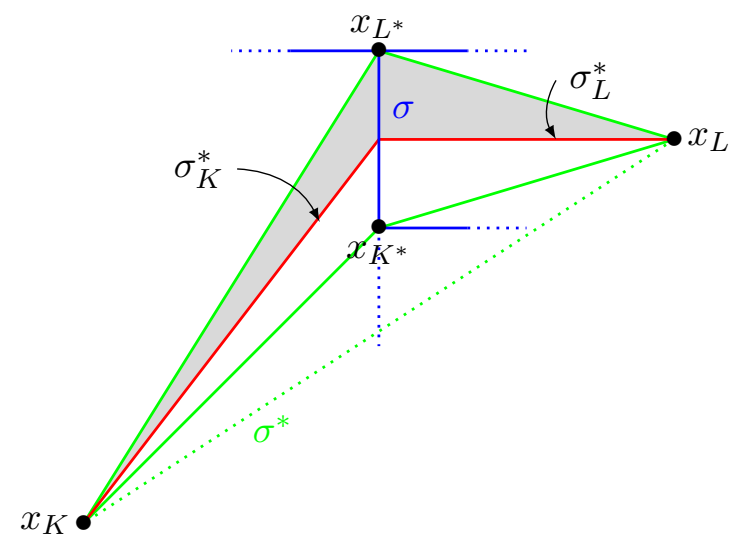

Figure 13: A case of non convex diamond cells: there are two edges of the dual mesh included in the diamond cell. The shaded area is $D_{\sigma, \sigma^{*}} \cap L^{*}$

Let us detail how the construction of the mass fluxes $\mathcal{F}_{K, \sigma}$ and $\mathcal{F}_{K^{*}, \sigma^{*}}$ made in Section 3.2 generalizes for the barycentric dual mesh. We refer the reader to Fig. 13 again. As in Section 3.2, Lemma 3.3 gives the existence of a function $\omega_{D_{\sigma, \sigma^{*}}} \in H_{\mathrm{div}}$ such that

$$
\nabla \cdot \omega_{D_{\sigma, \sigma^{*}}}=\frac{1}{\left|D_{\sigma, \sigma^{*}}\right|} \sum_{\mathfrak{s} \in \partial D_{\sigma, \sigma^{*}}}|\mathfrak{s}| \mathcal{F}_{D_{\sigma, \sigma^{*}, \mathfrak{s}}}
$$

and

$$
\int_{\mathfrak{s}} \omega_{D_{\sigma, \sigma^{*}}} \cdot \mathbf{n}_{D_{\sigma, \sigma^{*}, \mathfrak{s}}}=|\mathfrak{s}| \mathcal{F}_{D_{\sigma, \sigma^{*}, \mathfrak{s}}}
$$

There is no restriction at all on the geometry of the cells, in defining a mass flux on an edge $\mathfrak{r}$ (of $X=K$ or $X=K^{*}$ ) contained in $D_{\sigma, \sigma^{*}}$, with the unit outward normal $\mathbf{n}_{X, \mathfrak{r}}$ by the formula

$$
|\mathfrak{r}| \mathcal{F}_{X, \mathfrak{r}}=\int_{\mathfrak{r}} \omega_{D_{\sigma, \sigma *}} \cdot \mathbf{n}_{X, \mathfrak{r}} \cdot
$$

This is still a conservative quantity: if $\mathfrak{r}=X \mid Y \subset D_{\sigma, \sigma^{*}}$, then $\mathcal{F}_{X, \mathfrak{r}}=-\mathcal{F}_{Y, \mathfrak{r}}$, and we adopt this definition from now on. The domain $D_{\sigma, \sigma^{*}} \cap L^{*}$ is delimited by $\sigma_{K}^{*}, \sigma_{L}^{*}$ (which are not necessarily on the same direction), $\mathfrak{s}_{K L^{*}}$, and $\mathfrak{s}_{L^{*} L}$. The mass fluxes are already known on $\mathfrak{s}_{K L^{*}}$, and $\mathfrak{s}_{L^{*} L}$, which are boundaries of the diamond $D_{\sigma, \sigma^{*}}$, and we wish to clarify their expression on $\sigma_{K}^{*}, \sigma_{L}^{*}$, the boundaries of $L^{*}$. Therefore, by definition of $\omega_{D_{\sigma, \sigma^{*}}}$ and the divergence theorem, we obtain

$$
\begin{aligned}
\int_{D_{\sigma, \sigma^{*}} \cap L^{*}} \nabla \cdot \omega_{D_{\sigma, \sigma^{*}}}= & \frac{\left|D_{\sigma, \sigma^{*}} \cap L^{*}\right|}{\left|D_{\sigma, \sigma^{*}}\right|} \sum_{\mathfrak{s} \in \partial D_{\sigma, \sigma^{*}}}|\mathfrak{s}| \mathcal{F}_{D_{\sigma, \sigma^{*}, \mathfrak{s}}} \\
= & \left|\sigma_{K}^{*}\right| \mathcal{F}_{L^{*}, \sigma_{K}^{*}}+\left|\sigma_{L}^{*}\right| \mathcal{F}_{L^{*}, \sigma_{L}^{*}} \\
& +\left|\mathfrak{s}_{K L^{*}}\right| \mathcal{F}_{D_{\sigma, \sigma^{*}, \mathfrak{s}_{K} L^{*}}}+\left|\mathfrak{s}_{L^{*} L}\right| \mathcal{F}_{D_{\sigma, \sigma^{*}, \mathfrak{s}_{L^{*} L}}}
\end{aligned}
$$

We write

$$
1=\frac{\left|D_{\sigma, \sigma^{*}} \cap L^{*}\right|}{\left|D_{\sigma, \sigma^{*}}\right|}+\frac{\left|D_{\sigma, \sigma^{*}} \cap K^{*}\right|}{\left|D_{\sigma, \sigma^{*}}\right|}
$$


so that

$$
\begin{aligned}
& \left|\sigma_{K}^{*}\right| \mathcal{F}_{L^{*}, \sigma_{K}^{*}}+\left|\sigma_{L}^{*}\right| \mathcal{F}_{L^{*}, \sigma_{L}^{*}}=\frac{\left|D_{\sigma, \sigma^{*}} \cap L^{*}\right|}{\left|D_{\sigma, \sigma^{*}}\right|} \sum_{\mathfrak{s} \in \partial D_{\sigma, \sigma^{*}}}|\mathfrak{s}| \mathcal{F}_{D_{\sigma, \sigma^{*}, \mathfrak{s}}}
\end{aligned}
$$

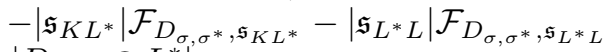

$$
\begin{aligned}
& =\frac{\left|D_{\sigma, \sigma^{*}} \cap L^{*}\right|^{\prime}}{\left|D_{\sigma, \sigma^{*}}\right|} \sum_{\mathfrak{s} \in \partial D_{\sigma, \sigma^{*}} \cap K^{*}}|\mathfrak{s}| \mathcal{F}_{D_{\sigma, \sigma^{*}, \mathfrak{s}}} \\
& -\frac{\left|D_{\sigma, \sigma^{*}} \cap K^{*}\right|}{\left|D_{\sigma, \sigma^{*}}\right|} \sum_{\mathfrak{s} \in \partial D_{\sigma, \sigma^{*}} \cap L^{*}}|\mathfrak{s}| \mathcal{F}_{D_{\sigma, \sigma^{*}, \mathfrak{s}}}
\end{aligned}
$$

We recover the same expression as for the direct mesh, and it makes sense to still denote this sum by $\mathcal{F}_{K^{*}, \sigma^{*}}$. It is worth pointing out that computing $\mathcal{F}_{K^{*}, \sigma^{*}}$ does not need to determine explicitly $\left|\sigma_{K}^{*}\right| \mathcal{F}_{L^{*}, \sigma_{K}^{*}}$ and $\left|\sigma_{L}^{*}\right| \mathcal{F}_{L^{*}, \sigma_{L}^{*}}$ : the sum can be evaluated directly form the knowledge of the mass fluxes on the interfaces of the diamond cells. Next, we can define the splitting $\mathcal{F}_{K^{*}, \sigma^{*}}=\mathcal{F}_{K^{*}, \sigma^{*}}^{+}+\mathcal{F}_{K^{*}, \sigma^{*}}^{-}$as we did for the primal mesh or the direct dual mesh, which leads to (16). It is worth detailing further this decomposition and explaining its consistency. Indeed, the auxilliary function $\omega_{D_{\sigma, \sigma^{*}}}$ depends on the fluxes $\mathcal{F}_{D_{\sigma, \sigma^{*}, \mathfrak{s}}}=\mathcal{F}_{D_{\sigma, \sigma^{*}, \mathfrak{s}}}^{+}+\mathcal{F}_{D_{\sigma, \sigma^{*}, \mathfrak{s}}}^{-}$, and, it turns out that $\mathcal{F}_{X, \mathfrak{r}}$, defined by (47) appears as a linear combination of these fluxes; we get

$$
\mathcal{F}_{X, \mathfrak{r}}=\sum_{\mathfrak{s} \in \partial D_{\sigma, \sigma^{*}}, D_{\sigma, \sigma^{*}} \in \mathfrak{D}_{X}} \eta_{\mathfrak{s}} \mathcal{F}_{D_{\sigma, \sigma^{*}, \mathfrak{s}}} .
$$

The expression of the coefficients is fully detailed in [23], but we dot not need this here: we point out that the $\eta_{\mathfrak{s}}$ are real, without definite sign; this explains why a construction applying directly Lemma 3.3 to the $\mathcal{F}_{D_{\sigma, \sigma^{*}, \mathfrak{s}}^{ \pm}}$would fail: it is not guaranteed that the combination of the $\mathcal{F}_{D_{\sigma, \sigma^{*}, \mathfrak{s}}}^{ \pm}$keeps the sign. Nevertheless, we can write

$$
\begin{aligned}
\mathcal{F}_{X, \mathfrak{r}}= & \underbrace{\sum_{\eta_{\mathfrak{s}} \geqslant 0} \eta_{\mathfrak{s}} \mathcal{F}_{D_{\sigma, \sigma^{*}, \mathfrak{s}}}^{+}+\sum_{\eta_{\mathfrak{s}} \leqslant 0} \eta_{\mathfrak{s}} \mathcal{F}_{D_{\sigma, \sigma^{*}, \mathfrak{s}}}^{-}}_{\geqslant 0} \\
& +\underbrace{\sum_{\eta_{\mathfrak{s}} \leqslant 0} \eta_{\mathfrak{s}} \mathcal{F}_{D_{\sigma, \sigma^{*}, \mathfrak{s}}^{+}}^{+}+\sum_{\eta_{\mathfrak{s}} \geqslant 0} \eta_{\mathfrak{s}} \mathcal{F}_{D_{\sigma, \sigma^{*}, \mathfrak{s}}}^{-}}_{\leqslant 0} .
\end{aligned}
$$

This defines the splitting $\mathcal{F}_{X, \mathfrak{r}}=\mathcal{F}_{X, \mathfrak{r}}^{+}+\mathcal{F}_{X, \mathfrak{r}}^{-}$. We apply this construction to define $\mathcal{F}_{K^{*}, \sigma_{K}^{*}}=$ $\mathcal{F}_{K^{*}, \sigma_{K}^{*}}^{+}+\mathcal{F}_{K^{*}, \sigma_{K}^{*}}^{-}$and $\mathcal{F}_{K^{*}, \sigma_{L}^{*}}=\mathcal{F}_{K^{*}, \sigma_{L}^{*}}^{+}+\mathcal{F}_{K^{*}, \sigma_{L}^{*}}^{-}$. By summing these contributions, we go back to

$$
\left|\sigma^{*}\right| \mathcal{F}_{K^{*}, \sigma^{*}}=\left|\sigma_{K}^{*}\right| \mathcal{F}_{K^{*}, \sigma_{K}^{*}}+\left|\sigma_{L}^{*}\right| \mathcal{F}_{K^{*}, \sigma_{L}^{*}}
$$

and then we obtain

$$
\left|\sigma^{*}\right| \mathcal{F}_{K^{*}, \sigma^{*}}^{ \pm}=\left|\sigma_{K}^{*}\right| \mathcal{F}_{K^{*}, \sigma_{K}^{*}}^{ \pm}+\left|\sigma_{L}^{*}\right| \mathcal{F}_{K^{*}, \sigma_{L}^{*}}^{ \pm}
$$

The proof of Proposition 3.5 can now be reproduced: the same formula holds for both the direct and the barycentric dual meshes, by merging contributions as in (48).

Next, we turn to the momentum equation, as in Section 3.3. For the barycentric dual mesh, there are two viewpoints:

*) either we define the mass fluxes on all interfaces of the dual mesh, by using the general formula (47). Then, on these interfaces, say $\sigma_{K}^{*}$, we split as described above the flux into positive and negative parts $\mathcal{F}_{K^{*}, \sigma_{K}^{*}}=\mathcal{F}_{K^{*}, \sigma_{K}^{*}}^{+}+\mathcal{F}_{K^{*}, \sigma_{K}^{*}}^{-}$, and we apply the upwinding 
principles accordingly by setting $\mathcal{G}_{K^{*}, \sigma_{K}^{*}}=\mathcal{F}_{K^{*}, \sigma_{K}^{*}}^{+} \mathbf{u}_{K^{*}}+\mathcal{F}_{K^{*}, \sigma_{K}^{*}}^{-} \mathbf{u}_{L^{*}}$. As in (48), we can merge the contributions $\left|\sigma_{K}^{*}\right| \mathcal{G}_{K^{*}, \sigma_{K}^{*}}$ and $\left|\sigma_{L}^{*}\right| \mathcal{G}_{K^{*}, \sigma_{L}^{*}}$ : the sum can be cast into

$$
\left(\left|\sigma_{K}^{*}\right| \mathcal{F}_{K^{*}, \sigma_{K}^{*}}^{+}+\left|\sigma_{L}^{*}\right| \mathcal{F}_{K^{*}, \sigma_{L}^{*}}^{+}\right) \mathbf{u}_{K^{*}}+\left(\left|\sigma_{K}^{*}\right| \mathcal{F}_{K^{*}, \sigma_{K}^{*}}^{-}+\left|\sigma_{L}^{*}\right| \mathcal{F}_{K^{*}, \sigma_{L}^{*}}^{+}\right) \mathbf{u}_{L^{*}}
$$

**) or, we use directly the averaged formula (48), which defines $\mathcal{F}_{K^{*}, \sigma^{*}}^{ \pm}$by (16) too, and then we make use of $(20)$.

The two viewpoints coincide, owing to (49).

Definition 3.7 of the pressure gradient can be readily adapted: the formula for $\left(\boldsymbol{\nabla}_{\boldsymbol{d}} p\right)_{K^{*}}$ still makes sense, by setting

$$
\left|\sigma^{*}\right| \mathbf{n}_{K^{*}, \sigma^{*}}=\left|\sigma_{K}^{*}\right| \mathbf{n}_{K^{*}, \sigma_{K}^{*}}+\left|\sigma_{L}^{*}\right| \mathbf{n}_{K^{*}, \sigma_{L}^{*}},
$$

remarking that this vector is indeed orthogonal to $\sigma^{*}$, by virtue of Lemma 2.4.

For obtaining the kinetic energy fluxes, as in Section 3.4, we can define the "intermediate" kinetic energy fluxes $\mathcal{K}_{K^{*}, \sigma_{K}^{*}}, \mathcal{K}_{K^{*}, \sigma_{L}^{*}}$ by going back to (49). It yields

$$
\begin{aligned}
& \mathcal{K}_{K^{*}, \sigma_{K}^{*}}=\left(\mathcal{F}_{K^{*}, \sigma_{K}^{*}}^{+} \frac{\left\|\mathbf{u}_{K^{*}}\right\|^{2}}{2}+\mathcal{F}_{K^{*}, \sigma_{K}^{*}}^{-} \frac{\left\|\mathbf{u}_{L^{*}}\right\|^{2}}{2}\right), \\
& \mathcal{K}_{K^{*}, \sigma_{L}^{*}}=\left(\mathcal{F}_{K^{*}, \sigma_{L}^{*}}^{+} \frac{\| \mathbf{u}_{K^{*} \|^{2}}}{2}+\mathcal{F}_{K^{*}, \sigma_{L}^{*}}^{-} \frac{\left\|\mathbf{u}_{L^{*}}\right\|^{2}}{2}\right), \\
& \left|\sigma^{*}\right| \mathcal{K}_{K^{*}, \sigma^{*}}=\left|\sigma_{K}^{*}\right| \mathcal{K}_{K^{*}, \sigma_{K}^{*}}+\left|\sigma_{L}^{*}\right| \mathcal{K}_{K^{*}, \sigma_{L}^{*}} .
\end{aligned}
$$

Proof of Lemma 3.12 still follows by using $-\mathbf{n}_{L, \sigma}=\mathbf{n}_{K, \sigma}$ and $-\mathbf{n}_{L^{*}, \sigma^{*}}=\mathbf{n}_{K^{*}, \sigma^{*}}$ owing to definition (50) for $\mathbf{n}_{K^{*}, \sigma^{*}}$.

It remains to discuss the conservation of total energy and to extend Proposition 4.2. When working with the barycentric mesh, we still apply Lemma 3.3 , but in order to define $\omega_{K^{*}}$, we should use the two quantities $\mathcal{X}_{K^{*}, \sigma_{K}^{*}}=\mathcal{K}_{K^{*}, \sigma_{K}^{*}}$ and $\mathcal{X}_{K^{*}, \sigma_{L}^{*}}=\mathcal{K}_{K^{*}, \sigma_{L}^{*}}$, see (51), associated to the two interfaces $\sigma_{K}^{*}$ and $\sigma_{L}^{*}$ of $K^{*}$ that belong to $D_{\sigma, \sigma^{*}}$. We next define a conservative flux of kinetic energy for each $\mathfrak{s}=\left[x_{K}, x_{K^{*}}\right] \in \partial D_{\sigma, \sigma^{*}}$ as follows

$$
\mathcal{K}_{D_{\sigma, \sigma^{*}, \mathfrak{s}}}=\frac{1}{2|\mathfrak{s}|} \int_{\mathfrak{s}}\left(\omega_{K}+\omega_{K^{*}}\right) \mathbf{n}_{D_{\sigma, \sigma^{*}, \mathfrak{s}}} \text { where } \mathfrak{s}=\mathfrak{s}_{K K^{*}}
$$

With a convenient reorganization of the terms, we write $\sum_{\mathfrak{s} \in \partial D_{\sigma, \sigma^{*}}}|\mathfrak{s}| \mathcal{K}_{D_{\sigma, \sigma^{*}, \mathfrak{s}}}$ as

$$
\begin{aligned}
& \frac{1}{2}\left(\int_{\mathfrak{s}_{K K^{*}}} \omega_{K} \mathbf{n}_{D_{\sigma, \sigma^{*}, \mathfrak{s}_{K} K^{*}}}+\int_{\mathfrak{s}_{K L^{*}}} \omega_{K} \mathbf{n}_{D_{\sigma, \sigma^{*}, \mathfrak{s}_{K L^{*}}}}\right) \\
& +\frac{1}{2}\left(\int_{\mathfrak{s}_{L K^{*}}} \omega_{L} \mathbf{n}_{D_{\sigma, \sigma^{*}, \mathfrak{s}_{L K^{*}}}}+\int_{\mathfrak{s}_{L L^{*}}} \omega_{L} \mathbf{n}_{D_{\sigma, \sigma^{*}, \mathfrak{s}_{L L^{*}}}}\right) \\
& +\frac{1}{2}\left(\int_{\mathfrak{s}_{K K^{*}}} \omega_{K^{*}} \mathbf{n}_{D_{\sigma, \sigma^{*}, \mathfrak{s}_{K K^{*}}}}+\int_{\mathfrak{s}_{L K^{*}}} \omega_{K^{*}} \mathbf{n}_{D_{\sigma, \sigma^{*}, \mathfrak{s}_{L K^{*}}}}\right) \\
& +\frac{1}{2}\left(\int_{\mathfrak{s}_{K L^{*}}} \omega_{L^{*}} \mathbf{n}_{D_{\sigma, \sigma^{*}, \mathfrak{s}_{K} L^{*}}}+\int_{\mathfrak{s}_{L L^{*}}} \omega_{L^{*}} \mathbf{n}_{D_{\sigma, \sigma^{*}, \mathfrak{s}_{L L^{*}}}}\right) .
\end{aligned}
$$

In the energy balance, we should modify (43) since we take into account the two interfaces $\sigma_{K}^{*}$ and $\sigma_{L}^{*}$; on the dual cell $K^{*}$ we now have

$$
\mathcal{K}_{K^{*}, \sigma_{K}^{*}}+\mathcal{K}_{L^{*}, \sigma_{K}^{*}}=0 \quad \text { and } \quad \mathcal{K}_{K^{*}, \sigma_{L}^{*}}+\mathcal{K}_{L^{*}, \sigma_{L}^{*}}=0 .
$$


Accordingly, (44) becomes

$$
\frac{1}{2}\left(\int_{\sigma_{K}^{*}} \omega_{K^{*}} \mathbf{n}_{K^{*}, \sigma_{K}^{*}}+\int_{\sigma_{K}^{*}} \omega_{L^{*}} \mathbf{n}_{L^{*}, \sigma_{K}^{*}}\right)=0,
$$

and

$$
\frac{1}{2}\left(\int_{\sigma_{L}^{*}} \omega_{K^{*}} \mathbf{n}_{K^{*}, \sigma_{L}^{*}}+\int_{\sigma_{L}^{*}} \omega_{L^{*}} \mathbf{n}_{L^{*}, \sigma_{L}^{*}}\right)=0 .
$$

We are thus led to

$$
\begin{aligned}
& \sum_{\mathfrak{s} \in \partial D_{\sigma, \sigma^{*}}}|\mathfrak{s}| \mathcal{K}_{D_{\sigma, \sigma^{*}, \mathfrak{s}}}=\frac{1}{2}\left(\int_{\sigma} \omega_{K} \mathbf{n}_{K, \sigma}+\int_{\mathfrak{s}_{K K^{*}}} \omega_{K} \mathbf{n}_{D_{\sigma, \sigma^{*}, \mathfrak{s}_{K} K^{*}}}+\int_{\mathfrak{s}_{K L^{*}}} \omega_{K} \mathbf{n}_{D_{\sigma, \sigma^{*}, \mathfrak{s}_{K L}}}\right) \\
& +\frac{1}{2}\left(\int_{\sigma} \omega_{L} \mathbf{n}_{L, \sigma}+\int_{\mathfrak{s}_{L K^{*}}} \omega_{L} \mathbf{n}_{D_{\sigma, \sigma^{*}, \mathfrak{s}_{L K^{*}}}}+\int_{\mathfrak{s}_{L L^{*}}} \omega_{L} \mathbf{n}_{D_{\sigma, \sigma^{*}, \mathfrak{s}_{L L^{*}}}}\right) \\
& +\frac{1}{2}\left(\int_{\sigma_{K}^{*}} \omega_{K^{*}} \mathbf{n}_{K^{*}, \sigma_{K}^{*}}+\int_{\sigma_{L}^{*}} \omega_{K^{*}} \mathbf{n}_{K^{*}, \sigma_{L}^{*}}\right. \\
& \left.+\int_{\mathfrak{s}_{K K^{*}}} \omega_{K^{*}} \mathbf{n}_{D_{\sigma, \sigma^{*}, \mathfrak{s}_{K} K^{*}}}+\int_{\mathfrak{s}_{L K^{*}}} \omega_{K^{*}} \mathbf{n}_{D_{\sigma, \sigma^{*}, \mathfrak{s}_{L K}}}\right) \\
& +\frac{1}{2}\left(\int_{\sigma_{K}^{*}} \omega_{L^{*}} \mathbf{n}_{L^{*}, \sigma_{K}^{*}}+\int_{\sigma_{L}^{*}} \omega_{L^{*}} \mathbf{n}_{L^{*}, \sigma_{L}^{*}}\right. \\
& \left.+\int_{\mathfrak{s}_{K L^{*}}} \omega_{L^{*}} \mathbf{n}_{D_{\sigma, \sigma^{*}, \mathfrak{s}_{K L^{*}}}}+\int_{\mathfrak{s}_{L L^{*}}} \omega_{L^{*}} \mathbf{n}_{D_{\sigma, \sigma^{*}, \mathfrak{s}_{L} L^{*}}}\right) .
\end{aligned}
$$

From this, we can apply the divergence theorem and conclude as for the direct mesh. The treatment of the pressure term follows similar arguments, up to the definition $\mathcal{X}_{K^{*}, \sigma_{K}^{*}}=$ $p_{\sigma, \sigma^{*}} \overline{\mathbf{u}}_{K^{*}} \cdot \mathbf{n}_{K^{*}, \sigma_{K}^{*}}, \mathcal{X}_{K^{*}, \sigma_{L}^{*}}=p_{\sigma, \sigma^{*}} \overline{\mathbf{u}}_{K^{*}} \cdot \mathbf{n}_{K^{*}, \sigma_{L}^{*}}$ and replacing (46) by the sum over the interfaces $\sigma_{K}^{*}$ and $\sigma_{L}^{*}$.

Acknowledgments. The authors want to express their gratitude to Raphaël Loubère for fruitful discussions and to thank the referees for their careful reading of the paper and their valuable remarks.

Data availability statement. The data that support the findings of this study are available from the corresponding author upon reasonable request

\section{References}

[1] R. Abgrall, K. Lipnikov, N. Morgan, and S. Tokareva. Multidimensional staggered grid Residual Distribution scheme for Lagrangian hydrodynamics. SIAM J. Sci. Comput., 42(1):343-A370, 2020.

[2] R. Abgrall and P. Roe. High order fluctuation schemes on triangular meshes. J. Sci. Comput., 19:3-36, 2003.

[3] N. Aguillon. Problèmes d'interfaces et couplages singuliers dans les systèmes hyperboliques: analyse et analyse numérique. PhD thesis, Univ. Paris Sud, 2014.

[4] G. Ansanay-Alex, F. Babik, J.-C. Latché, and D. Vola. An $L^{2}$-stable approximation of the Navier-Stokes convection operator for low-order non-conforming finite elements. Int. J. Numer. Meth. Fluids, 66:555-580, 2011. 
[5] F. Berthelin, T. Goudon, and S. Minjeaud. Consistency analysis of a 1D finite volume scheme for barotropic Euler models. In J. Fuhrmann, M. Ohlberger, and C. Rohde, editors, Finite Volumes for Complex Applications VII; Methods, Theoretical Aspects, and Elliptic, Parabolic and Hyperbolic Problems, Berlin, volume 77 and 78 of Springer Proceedings in Mathematics \& Statistics, pages 97-106. Springer, 2014.

[6] F. Berthelin, T. Goudon, and S. Minjeaud. Kinetic schemes on staggered grids for barotropic Euler models: entropy-stability analysis. Math. Comput., 84:2221-2262, 2015.

[7] F. Berthelin, T. Goudon, and S. Minjeaud. Multifluid flows: a kinetic approach. J. Sci. Comput., 66(2):792-824, 2016.

[8] C. Berthon, Y. Coudière, and V. Desveaux. Second-order MUSCL schemes based on dual mesh gradient reconstruction (DMGR). ESAIM Math. Model. Numer. Anal., 48(2):583602, 2014.

[9] F. Boyer, S. Krell, and F. Nabet. Inf-Sup Stability of the Discrete Duality Finite Volume method for the 2D Stokes problem. Mathematics of Computation, 84:2705-2742, 2015.

[10] C. Calgaro, E. Chane-Kane, E. Creusé, and T. Goudon. $L^{\infty}$-stability of vertex-based MUSCL finite volume schemes on unstructured grids: simulation of incompressible flows with high density ratios. J. Comput. Phys., 229(17):6027-6046, 2010.

[11] C. Calgaro, E. Creusé, T. Goudon, and S. Krell. Simulations of non homogeneous viscous flows with incompressibility constraints. Math. Comput. Simul., 137:201-225, 2017.

[12] G. Dakin, B. Després, and S. Jaouen. High-order staggered schemes for compressible hydrodynamics. weak consistency and numerical validation. J. Comput. Phys., 376:339364, 2019.

[13] S. Delcourte. Développement de méthodes de volumes finis pour la mécanique des fluides. PhD thesis, Université Paul Sabatier, Toulouse, France, 2007.

[14] S. Delcourte and P. Omnes. A discrete duality finite volume discretization of the vorticityvelocity-pressure formulation of the 2D Stokes problem on almost arbitrary two-dimensional grids. Numer. Meth. for PDEs, 31(1):1-30, 2015.

[15] S. Dellacherie. Analysis of Godunov type schemes applied to the compressible Euler system at low Mach number. J. Comput. Phys., 229(4):978-1016, 2010.

[16] S. Dellacherie, P. Omnes, and F. Rieper. The influence of cell geometry on the Godunov scheme applied to the linear wave equation. J. Comput. Phys., 229:5315-5338, 2010.

[17] B. Després. Lois de conservation eulériennes, lagrangiennes et méthodes numériques, volume 68 of Math et Appl. Springer, 2010.

[18] K. Domelevo and P. Omnes. A finite volume method for the Laplace equation on almost arbitrary two-dimensional grids. ESAIM Math. Model. Numer. Anal., 39(6):1203-1249, 2005.

[19] T. Gallouët, R. Herbin, and J.-C. Latché. On the weak consistency of finite volume schemes for conservation laws on general meshes. SeMA Journal, 76(4):581-594, 2019.

[20] T. Gallouët, R. Herbin, and J.-C. Latché. Weak consistency of finite volume schemes for systems of non linear conservation laws: Extension to staggered schemes. working paper or preprint, Mar. 2021.

[21] T. Goudon and S. Krell. A DDFV scheme for incompressible Navier- Stokes equations with variable density. In J. Fuhrmann, M. Ohlberger, and C. Rohde, editors, Finite Volumes for Complex Applications VII-Elliptic, Parabolic and Hyperbolic Problems, pages 627-635. Springer International Publishing, 2014.

[22] T. Goudon, S. Krell, and G. Lissoni. DDFV method for navier-stokes problem with outflow boundary conditions. Numerische Mathematik, 142(1):55-102, 2019. 
[23] T. Goudon, S. Krell, J. Llobell, and S. Minjeaud. Transfer of conservative discrete differential operators between staggered grids: construction and duality relations. IMA J. Numer. Anal., 2021. To appear.

[24] T. Goudon, J. Llobell, and S. Minjeaud. An asymptotic preserving scheme on staggered grids for the barotropic Euler system in low Mach regimes. Numer Methods Partial Differential Eq., 36:1098-1128, 2020.

[25] T. Goudon, J. Llobell, and S. Minjeaud. An explicit MUSCL scheme on staggered grids with kinetic-like fluxes for the barotropic and full Euler system. Commun. Comput. Phys., $27: 672-724,2020$.

[26] J.-L. Guermond, B. Popov, and V. Tomov. Entropy-viscosity method for the single material Euler equations in lagrangian frame. Comput. Meth. Appl. Mech. Eng., 300:402-426, 2016.

[27] H. Guillard and A. Murrone. On the behavior of upwind schemes in the low Mach number limit. II: Godunov type schemes. Comput. Fluids, 33:655-675, 2004.

[28] H. Guillard and C. Viozat. On the behavior of upwind schemes in the low Mach number limit. Comput. Fluids, 28:63-86, 1999.

[29] F. H. Harlow and J. E. Welch. Numerical calculation of time-dependent viscous incompressible flow of fluid with free surface. Phys. Fluids, 8(12):2182-2189, 1965.

[30] R. Herbin, W. Kheriji, and J.-C. Latché. Staggered schemes for all speed flows. ESAIM:Proc, 35:122-150, 2012. Actes du Congrès National de Mathématiques Appliquées et Industrielles.

[31] R. Herbin, J.-C. Latché, S. Minjeaud, and N. Therme. Conservativity and weak consistency of a class of staggered finite volume methods for the Euler equations. Technical report, Aix-Marseille Univ., Univ. Côte d'Azur, CNRS, IRSN, CEA, Inria, 2020. Available at https://hal.archives-ouvertes.fr/hal-02308023v1.

[32] R. Herbin, J.-C. Latché, and T. T. Nguyen. Consistent explicit staggered schemes for compressible flows; part I: the barotropic Euler equations. Technical report, LATP, Univ. Aix-Marseille \& CNRS, 2013.

[33] R. Herbin, J.-C. Latché, and T. T. Nguyen. Explicit staggered schemes for the compressible Euler equations. In Applied mathematics in Savoie-AMIS 2012: Multiphase flow in industrial and environmental engineering, volume 40 of ESAIM Proc., pages 83-102. EDP Sci., Les Ulis, 2013.

[34] R. Herbin, J.-C. Latché, and T. T. Nguyen. Consistent segregated staggered schemes with explicit steps for the isentropic and full Euler equations. ESAIM Math. Model. Numer. Anal., 52(3):893-944, 2018.

[35] H. Hermeline. A finite volume method for the approximation of diffusion operators on distorted meshes. J.Comput.Phys, 160(2):481-499, 2000.

[36] J. Kamm. Evaluation of the Sedov-von Neumann-Taylor blast wave solution. Technical Report LA-UR-00-6055, Los Alamos National Laboratory, 2000.

[37] S. Kaniel and J. Falcovitz. Approximation of the hydrodynamic equations by a transport process. In R. Rautman, editor, Proceedings of IUTAM Symposium on Approximation Methods for Navier-Stokes Problems, volume 771 of Lecture Notes in Math. Springer-Verlag, 1980.

[38] S. Krell. Stabilized DDFV schemes for Stokes problem with variable viscosity on general 2D meshes. Numer. Meth.for PDEs, 27(6):1666-1706, 2011.

[39] S. Krell. Stabilized DDFV schemes for the incompressible Navier-Stokes equations. In Finite volumes for complex applications VI. Problems $\&$ perspectives. Volume 1, 2, volume 4 of Springer Proc. Math., pages 605-612. Springer, Heidelberg, 2011. 
[40] P. D. Lax and B. Wendroff. Systems of conservation laws. Comm. Pure Appl. Math., 13:217-237, 1960.

[41] F. Lespagnol and G. Dakin. High order accurate schemes for Euler and Navier-Stokes equations on staggered Cartesian grids. J. Comput. Phys., 410:109314, 2020.

[42] M.-S. Liou. A sequel to AUSM: AUSM+. J. Comput. Phys., 129:364-382, 1996.

[43] M.-S. Liou and C. J. Steffen Jr. A new flux splitting scheme. J. Comput. Phys., 107:23-39, 1993.

[44] G. Lissoni. DDFV for Navier-Stokes equations. PhD thesis, Univ. Côte d'Azur, 2019.

[45] J. Llobell. Schémas Volumes Finis à mailles décalées pour la dynamique des gaz. PhD thesis, Univ. Côte d'Azur, 2018.

[46] R. Loubère and M. Shashkov. A subcell remapping method on staggered polygonal grids for arbitrary-Lagrangian-Eulerian methods. J. Comput. Phys., 209(1):105-138, 2005.

[47] B. Perthame. Second order Boltzmann schemes for compressible Euler equations in one and two space dimension. SIAM J. Numer. Anal., 29(1):1-19, 1992.

[48] B. Perthame. Kinetic formulation of conservation laws. Oxford Lecture Series in Math. and its Appl. Oxford University Press, 2003.

[49] L. I. Sedov. Similarity and dimensional methods in mechanics. Academic Press, New York-London, 1959. Translation by Morris Friedman (translation edited by Maurice Holt).

[50] E. F. Toro. Riemann solvers and numerical methods for fluid dynamics. Springer-Verlag, Berlin, third edition, 2009.

[51] Y.-Y. Tsui and T.-C. Wu. A pressure-based unstructured-grid algorithm using highresolution schemes for all-speed flows. Numerical Heat Transfer, Part B: Fundamentals, $53(1): 75-96,2008$.

[52] J. von Neumann and R. D. Richtmyer. A method for the numerical calculation of hydrodynamics shocks. J. Appl. Phys., 21:232-237, 1950. 